\title{
RECURSIVELY ENUMERABLE DEGREES AND THE CONJUGACY PROBLEM
}

BY

\author{
DONALD J. COLLINS
}

Queen Mary College, London, England(1)

The principal result obtained is the theorem that for every recursively enumerable degree of unsolvability, there exists a finitely presented group whose conjugacy problem has that degree. (Parts I, II, III and IV.) In Part V this result is generalised to the theorem that certain complexes of recursively enumerable degrees of unsolvability may be obtained as the degrees of a complex of problems concerning conjugacy in a finitely presented group.

It is a pleasure to acknowledge the encouragement and inspiration provided by Professor William Boone during this work.

\section{Introduction}

In 1911, Max Dehn formulated three fundamental decision problems $\left({ }^{2}\right)$ concerning groups: the word problem, the conjugacy (or transformation) problem and the isomorphism problem. These may be roughly stated as: $\left(^{3}\right)$ (i) Word problem for the group $G$-does there exist an effective method to determine of an arbitrary element $W$ of $G$ whether or not $W=1$ in $G$. (ii) Conjugacy problem for the group $G$-does there exist an effective method to determine of two arbitrary elements $U$ and $V$ of $G$ whether or not $U$ is conjugate to $V$ in $G$. (iii) Isomorphism problem for the class $C$ of groups--does there exist an effective method to determine of two arbitrary members $G_{1}$ and $G_{2}$ of $C$ whether or not $G_{1}$ is isomorphic to $G_{2}$. Dehn's principal goal was the formulation of algorithms to provide effective

(i) The material in this paper is taken from the author's Ph. D. thesis submitted to Princeton University.

${ }^{2}$ ) A decision problem is a problem of the following type. Let $C$ be a class of entities and $P$ a property such that every $n$-tuple (where $n$ is fixed) of elements of $C$ either does or does not enjoy $P$. Does there exist an effective procedure to determine of an arbitrary $n$-tuple $\left(a_{1}, a_{2}, \ldots, a_{n}\right)$ whether or not $\left(a_{1}, a_{2}, \ldots, a_{n}\right)$ enjoys $P$ ?

$\left({ }^{3}\right)$ A more careful statement would specify presentation of a group rather than group. 
solutions for these problems. The discovery in the 1930 's of recursively unsolvable decision problems, however, led mathematicians to investigate, with a view toward proving recursive unsolvability, decision problems for which recursive solutions had previously been sought.

The first such result regarding Dehn's problems was obtained in 1954 by Novikov [18] who proved that there exists a group whose conjugacy problem is recursively unsolvable. Shortly thereafter Novikov [19] and Boone [4] each exhibited a finitely presented group with recursively unsolvable word problem. Finally, it was proved by Adjan [1] and Rabin [20], independently, that the isomorphism problem for the class of all groups was recursively unsolvable.

At about the same time as the work of Adjan and Rabin, a new developement occurred in the theory of recursive unsolvability. A decision problem is called recursively enumerable (r.e.) if, roughly, it is possible to enumerate in some mechanical fashion either all the $n$-tuples for which the problem would be decided in the affirmative or all those for which it would be decided in the negative. (Almost all significant problems outside foundations are recursively enumerable.) Two decision problems are said to have the same degree of unsolvability if a recursive solution of the first provides a recursive solution of the second and conversely. Friedberg [13] and Muenik [17] (also independently) proved that there exist unsolvable recursively enumerable decision problems of distinct degrees of unsolvability. Since Dehn's decision prolems are recursively enumerable, it was natural to consider the question of whether or not there existed, for every recursively enumerable degree of unsolvability, an example of one of Dehn's problems which was unsolvable of that degree. The first such result was obtained by Fridman [12] who proved that for every r.e. degree of unsolvability there exists a finitely presented group whose word problem is of that degree. This result was also proved by Bokut' [2], Boone [7] and Clapham [10]. Then Boone [8] proved that for every r.e. degree of unsolvability, there exists a class of finite presentations of groups whose isomorphism problem is of that degree. Our principal result rounds out this investigation. We prove that for every r.e. degree of unsolvability there exists a finitely presented group whose conjugacy problem is of that degree. An analogous result was obtained, more or less simultaneously, by Miller [15] for finitely generated, recursively presented groups.

Given that an r.e. degree can be considered as the degree of the word problem of a certain group or as the degree of the conjugacy problem of a certain group, it seems reasonable then to examine the relationship of the word and conjugacy problems for groups in general. The first result in this area was obtained by Fridman [11]. By proving that there existed a group with solvable word problem and unsolvable conjugacy problem, Fridman 
showed that the word and conjugacy problems are not always of the same degree. This has also been proved by Bokut' [3]. Since the groups we construct all have solvable word problems we prove a degree analogue of this result of Fridman. More generally one might ask whether or not any two r.e. degrees can be obtained as the degree of the word and conjugacy problems of a certain group. This question can be immediately answered in the negative since the word problem for a group must always be reducible to the conjugacy problem for the group. But there exist incomparable pairs of degrees. However, if the question is asked of any two degrees $D_{1}, D_{2}$ such that $D_{1}<D_{2}$ the answer is less immediate. Miller [16] has given an affirmative answer for finitely generated, recursively presented groups. At present we can only conjecture that the answer for finitely presented groups is also in the affirmative.

While the principal goal of the present paper is to obtain every r.e. degree as the degree of the conjugacy problem of a certain group, the method of proof is such that we are able to prove a result rather more general than this. Given a group $G$ and an element $V$ of $G$, the individual conjugacy problem for $V$ in $G$ is to determine of an arbitrary element $U$ of $G$ whether or not $U$ is conjugate to $V$ in $G$. The generalised theorem then states that given any recursively enumerable class $\left\{D_{n}\right\}$ of recursively enumerable degrees and any recursively enumerable degree $D \geqslant \operatorname{each} D_{n}$, there exists a group $G$ such that the set of degrees of the individual conjugacy problems for elements of $G$ consists exactly of all finite unions of the members of $\left\{D_{n}\right\}$ and the overall conjugacy problem for $G$ has a degree $D$. This theorem is an analogue of a theorem of Shepherdson [21] concerning individual word problems and overall word problem for a Thue system. (Moreover, we depend on Shepherdson's theorem to obtain our own theorem.)

\section{Some general remarks}

This paper has been written on the assumption that the reader has some familiarity with the papers [7] and [9], by Boone and Britton respectively. A complete knowledge of these two papers is not required. The reader should however be familiar with the definitions and lemmas (but not necessarily their proofs) of $\S 1$ of [9] and also of $\S 2$ of [7]. No knowledge of Shepherdson's [21] is assumed.

In the introduction to [7], Boone remarks that throughout his whole argument, the only tools employed are the Lemmas 3 and 4 proved by Britton in [9]. In a very real sense, the same is true of our argument. We do indeed develop other lemmas of a general nature but they are proved by using these two lemmas of Britton. Of these new lemmas, General Lemmas 3 and 4 (see Part I) are the most important. Indeed General Lemma 3 
may be fairly regarded as a kind of conjugacy analogue of Britton's Lemma 4 . This is well illustrated by a combinatorial approach to the word and conjugacy problems.

Let $E$ be a basis of $E^{*}$ with stable letters $\left\{p_{v}\right\}$ (see [9] for the definitions of all terms used here), say $E=(S ; D), E^{*}=\left(S,\left\{p_{v}\right\} ; D, F_{i} p_{v_{i}} G_{i}=H_{i} p_{v_{i}} K_{i}\right)$ and suppose the isomorphism condition holds. Let $U$ and $V$ be words of $E^{*}$ containing one or more positive $p_{v}$-symbols but no negative $p_{v}$-symbols. Then $U=V$ in $E^{*}$ if there exists a sequence $W_{1} \rightarrow W_{2} \rightarrow \ldots \rightarrow W_{n}$ where $U$ is $W_{1}$ and $V$ is $W_{n}$ and $W_{k} \rightarrow W_{k+1}$ either by application of a defining relation or by insertion or deletion of a pair of inverse generators. Britton's Lemma 4 (essentially) asserts that if such a sequence can be found, then there is a sequence of the same type in which a $p_{v}^{-1}$-symbol never appears. But there is a very similar combinatorial approach to conjugacy. Instead of regarding $U$ and $V$ as linear words, consider them as circular words (i.e. instead of the symbols being strung out in a line, they are wrapped around a drum). Then $U$ is conjugate to $V$ in $E^{*}$ if there exists a sequence of circular words $W_{1} \rightarrow W_{2} \rightarrow \ldots \rightarrow W_{n}$ where $U$ is $W_{1}, V$ is $W_{n}$ and $W_{k} \rightarrow W_{k+1}$ either by an application of a defining relation or by insertion or deletion of an inverse pair of generators, where these operations can be performed upon any section of the circular word. (In terms of our physical imagery it is clear that the drum must be expandable.) General Lemma 3 proves that if such a sequence exists, then there exists a sequence of the same type in which a $p_{v}^{-1}$. symbol never appears. A conjugacy analogue of Britton's Lemma 3 is impossible; for let $E=(a, b ; \varnothing)$ and $E^{*}=(a, b, p ; a p=p b)$. Then $a$ is conjugate to $b$ in $E^{*}$ but not in $E$.

In analysing the conjugacy problem for a group presentation, with a view toward obtaining either a recursive or a relatively recursive solution, there are two rather obvious approaches. One may attempt to show that it is necessary to solve the problem for a limited subclass of words rather than for all words. Alternatively one may attempt to show that only a limited subclass of words need be considered as possible conjugating elements. And of course, one can, as we do, combine these approaches. Roughly, we follow the first method for a while (Reductions II and IX) and then revert to the second (Reductions III and X). The main tool for the second approach is General Lemma 3 which shows that only a very restricted class of words need be considered as possible conjugating elements. Under certain circumstances, General Lemma 4 can be used to provide a considerable further restriction (Reductions IV and $\mathrm{X}$ ).

The plan of the argument is outlined in the diagrams on pp. 147, 151 and 155 . We use the "questionmark" notation defined in the middle of p. 533 of [6]. The diagrams are then to be interpreted in the following manner: a decision problem $P$ in the diagram is reducible to the collection of problems to which it is connected by an outward arrow. At various times during the argument we shall appear to consult oracles to determine 
whether or not there exist words of a certain type satisfying certain conditions and assume that an example is supplied along with an affirmative answer. But in fact this seemingly oracular consultation appears rather as an expositional device and questions of this type will be recursively solvable and we shall specify a recursive method to compute such a word if indeed one exists. (Actually this example "proviso" is not a restriction on genuine oracles-see the third paragraph on p. 53 of [7].)

\section{Statement of results}

RESULT A. Given any recursively enumerable degree of unsolvability $D$, there is a uniform explicit construction which yields a finitely presented group $G_{D}$ such that

(1) $G_{D}$ has solvable word problem;

(2) $G_{D}$ has conjugacy problem of degree $D$.

For a group presentation $G$, define the individual conjugacy problem for the word $V$ in $G$ to be the problem (? $U$, a word of $G)(\exists W) W^{-1} U W={ }_{G} V$. With this definition we can assert the following generalisation of Result $\mathrm{A}$.

RESULT B. Let $\left\{D_{n}\right\}$ be an r.e. set of r.e. degrees and $D$ an r.e. degree such that $D \geqslant D_{n}$ for every $n$. Then there exists a recursive construction which yields a finitely presented group $G\left(\left\{D_{n}\right\}, D\right)$ such that

(1) $G\left(\left\{D_{n}\right\}, D\right)$ has solvable word problem;

(2) for each $D_{n}$, there is a word $V_{n}$ such that the individual conjugacy problem for $V_{n}$ has degree $D_{n}$;

(3) $G\left(\left\{D_{n}\right\}, D\right)$ has conjugacy problem of degree $D$;

(4) the set of degrees of individual conjugacy problems of $G\left(\left\{D_{n}\right\}, D\right)$ consists of all finite joins $D_{i_{1}} \cup D_{i_{2}} \cup \ldots \cup D_{i_{m}}$ where $D_{i_{k}} \in\left\{D_{n}\right\}$ (including degree 0 as the empty join).

The starting point of our work is a theorem due to Shepherdson [21] which we state below. We shall, however, modify Shepherdson's terminology. For any Thue system $\mathfrak{T}$, the individual word problem for the word $\Phi_{0}$ in $\mathfrak{T}$ is the problem (? $\Phi$, a word of $\left.\mathfrak{T}\right) \Phi=\mathfrak{T} \Phi_{0}$. (Shepherdson calls this a special word problem.)

SHEPHERDSON's RESULT. Let $\left\{D_{n}\right\}$ be an r.e. set of r.e. degrees and $D$ any r.e. degree such that $D \geqslant D_{n}$ for every $n$. Then there exists a recursive construction which yields a Thue system $\mathfrak{I}\left(\left\{D_{n}\right\}, D\right)$ such that

(1) for every $D_{n}$, there exists a word $\Phi_{n}$ of $\mathfrak{I}\left(\left\{D_{n}\right\}, D\right)$ such that the individual word problem for $\Phi_{n}$ in $\mathfrak{I}\left(\left\{D_{n}\right\}, D\right)$ has degree $D_{n}$;

(2) the word problem for $\mathfrak{I}\left(\left\{D_{n}\right\}, D\right)$ has degree $D$; 
(3) the set of degrees of individual word problems of $\mathfrak{T}\left(\left\{D_{n}\right\}, D\right)$ consists of all finite joins $D_{i_{1}} \cup D_{i_{\mathrm{s}}} \cup \ldots \cup D_{i_{m}}$ where $D_{i_{k}} \in\left\{D_{n}\right\}$ (including degree 0 as the empty join);

(4) if the defining relations of $\mathfrak{T}\left(\left\{D_{n}\right\}, D\right)$ are $P_{i}=Q_{i}, i=1,2, \ldots, N$, then $i \neq j$ implies that $P_{1}$ is distinct from $P_{j}$ and $Q_{\mathfrak{t}}$ is distinct from $Q_{j}$;

(5) for each $i, P_{i}$ and $Q_{i}$ are at least two symbol occurrences long.

Parts (1)-(3) are explicitly stated in Theorem X of [21] and (4) and (5), which we require for technical reasons, are readily verified by inspection.

Before stating our main technical theorem, we must give a corollary to Shepherdson's theorem. Let $\mathfrak{I}\left(\left\{D_{n}\right\}, D\right)$ be presented as $\left(s_{1}, s_{2}, \ldots, s_{M} ; P_{i}=Q_{i}, i=1,2, \ldots, N\right)$. Then let $\mathfrak{T}_{*}\left(\left\{D_{n}\right\}, D\right)$ be the Thue system with presentation

$$
\left(s_{1}, s_{2}, \ldots, s_{M}, q ; P_{i} q=q Q_{i}, s_{b} q=q s_{b}, i=1,2, \ldots, N, b=1,2, \ldots, M\right)
$$

For notational convenience we write the defining relations of $\mathfrak{T}_{*}\left(\left\{D_{n}\right\}, D\right)$ as $F_{i} q=q K_{t}$, $i=1,2, \ldots, M+N$. A word of $\mathfrak{T}_{*}\left(\left\{D_{n}\right\}, D\right)$ is called special if it contains exactly one $q$-symbol.

Corolla $\mathrm{Y}$. Let $\left\{D_{n}\right\}$ and $D$ be as above. Then

(1) for each $D_{n}$, there exists a special word $\Omega_{n} q \Phi_{n}$ such that the problem (? $\Omega q \Phi$, a special word) $\Omega q \Phi=\Omega_{n} q \Phi_{n}$ in $\mathfrak{T}_{*}\left(\left\{D_{n}\right\}, D\right)$ has degree $D_{n}$;

(2) the problem (? $\Delta q \Pi, \Omega q \Phi$, special words) $\Delta q \Pi=\Omega q \Phi$ in $\mathfrak{I}_{*}\left(\left\{D_{n}\right\}, D\right)$ has degree $D$;

(3) the set of degrees of individual word problems of $\mathfrak{I}_{*}\left(\left\{D_{n}\right\}, D\right)$, where both the fixed word and the variable word are required to be special, consists of all finite joins $D_{i_{2}} \cup \ldots \cup D_{i_{m}}$ where $D_{i k_{c}} \in\left\{D_{n}\right\}$ (including degree 0 as the empty join);

(4) if $i \neq j$, then $F_{i}$ is distinct from $F_{j}$ and $K_{i}$ is distinct from $K_{j}$.

The corollary is most easily shown by observing that $\Delta q \Pi=\Omega q \Phi$ in $\mathfrak{T}_{*}\left(\left\{D_{n}\right\}, D\right)$ if and only if $\Delta \Pi=\Omega \Phi$ in $\mathfrak{T}\left(\left\{D_{n}\right\}, D\right)$. (The necessary argument is easily derived from the proof of Theorem 10 on p. 260 of [5].) Parts (1)-(3) then follow easily from parts (1)-(3) of Shepherdson's result. Part (4) is a consequence of parts (4) and (5) of Shepherdson's result.

We are now in a position to state the main technical theorem whose proof occupies most of the remainder of the paper.

Technical Result. Let $\mathfrak{I}_{*}$ be the Thue system with presentation $\left(s_{1}, s_{2}, \ldots, s_{M}, q\right.$; $\left.F_{i} q=q K_{i}, i=1,2, \ldots, P\right)$ where $F_{i}, K_{i}$ are $q$-free words such that assertion (4) of the corollary holds. Let $G_{0}$ be the group presentation 


$$
\begin{gathered}
s_{1}, s_{2}, \ldots, s_{M}, q, k, t, x, r_{i} \\
x s_{b}=s_{b} x x \quad r_{i} s_{b}=s_{b} x r_{i} x \\
r_{i} \bar{F}_{i} q=q K_{i} r_{i} \\
t x=x t \quad t r_{i}=r_{i} t \\
k x=x k \quad k r_{i}=r_{i} k
\end{gathered}
$$

where $i=1,2, \ldots, P, \quad b=1,2, \ldots, M$ and $\bar{F}_{i}$ is obtained from $F_{i}$ by replacing each occurrence of $s_{b}$ by $s_{b}^{-1}$ (e.g. $F_{i}$ is $s_{1} s_{2} s_{3}$ means $\bar{F}_{i}$ is $s_{1}^{-1} s_{2}^{-1} s_{3}^{-1}$ ). Then

(1) $G_{0}$ has solvable word problem;

(2) the conjugacy problem for $G_{0}$ is Turing equivalent to the word problem for special words of $\mathfrak{I}_{*}$.

As it stands the Technical Result, taken with the corollary, suffices only to prove Result A. However, once the proof of the Technical Result is completed, it is easy to indicate the fuller argument necessary to obtain Result $B$.

It should be mentioned that if Result A is all that is desired, then a theorem of Boone [6] may be taken as a starting-point. Boone's result asserts the existence of a Thue system whose word problem has arbitrary preassigned degree. However, the Thue system of Boone does not satisfy the technical conditions (4) and (5) of Shepherdson's result so that a certain amount of manipulation and construction must be carried out before a Thue system of the type $\mathfrak{I}_{*}$ in the Technical Result can be obtained. This manipulation and construction is not difficult and there seems to be little point to including it here.

\section{Part I}

The notation employed in this Part is almost identical to that used in $\S 2$ of [7]. Variations will be specifically noted; also we shall write $U$ is $V$ to mean that $U$ and $V$ are identical words.

Let $S$ be a set of generators and $\left\{A_{i}\right\},\left\{B_{i}\right\}, i \in I$, sets of words over $S .\left(^{1}\right)$ We use $A$ and $B$ as variables for formal products $A_{i_{1}}^{d_{1}} A_{i_{2}}^{d_{3}} \ldots A_{i_{m}}^{d_{m}}$ and $B_{j_{1}}^{e_{1}} B_{j_{2}}^{e_{2}} \ldots B_{f_{n}}^{e_{n}}$ respectively, where $d_{k}= \pm 1, e_{k}= \pm 1$ and $i_{k} \in I, j_{k} \in I$.

Let $A$ be $A_{i_{1}}^{d_{1}} A_{i_{2}}^{d_{2}} \ldots A_{i_{m}}^{d_{m}}$; if there exists $k$ such that $i_{k}=i_{k+1}$ and $d_{k}+d_{k+1}=0$, then a primitive $\alpha$-reduction of $A$ is $A_{i_{1}}^{d_{1}} A_{i_{2}}^{d_{z}} \ldots A_{i_{k-1}}^{d_{k-1}} A_{i_{k+2}}^{d_{k+2}} \ldots A_{i_{m}}^{d_{m}}$. A word $A$ is called $\alpha$-reduced

(1) The situation where we have two such sets of words will occur frequently. Our usual practice will be to analyse or make definitions with reference to one set and dismiss the other by remarking that the corresponding analysis or definition is similar (or dual). The reader should always be able to supply the second analysis or definition himself. 
if no primitive $\alpha$-reductions are applicable to it. An identical definition is given for words $B$. It is clear that beginning from any word $A$ or $B$ one can compute a finite sequence of primitive $\alpha$-reductions which terminates in an $\alpha$-reduced word. Moreover it is well known that any two such sequences beginning with the same word will terminate in the same word. We shall write $\alpha[A]$ or $\alpha[B]$ for this terminal word.

Finally if $m=n$ and, for all $k, i_{k}=j_{k}$ and $d_{k}=e_{k}$, we write $A \sim B$.

We vary the definition of primitive p-reduction which we choose to give as: if $W$ is a word of $E^{*}$, then

(i) if $W$ is $W_{1} p_{v}^{-1} C p_{v} W_{2}$ and $(\exists A)\left[C={ }_{E} A\right.$ and $\left.A \in A(v)\right]$ then $W_{1} \alpha[B] W_{2}$, where $A \sim B$, is a primitive $p$-reduction of $W$ with respect to $\left(E^{*}, E\right)$ (w.r.t. $\left(E^{*}, E\right)$ );

(ii) if $W$ is $W_{1} p_{v} C p_{v}^{-1} W_{2}$ and $(\exists B)\left[C={ }_{E} B\right.$ and $\left.B \in B(v)\right]$ then $W_{1} \alpha[A] W_{2}$, where $A \sim B$, is a primitive $p$-reduction of $W$ (w.r.t. $\left(E^{*}, E\right)$ ).

It is expositionally convenient to adopt this definition. All results proved by Boone in $\S 2$ of [7] remain valid for this altered definition. The phrase "with respect to $\left(E^{*}, E\right)$ " is added since we shall at times consider a certain set of letters to be stable for two pairs of groups. When no ambiguity can arise, it will be omitted.

Let $W$ be a word of $E^{*}$; (i) if $W_{1}$ is a primitive $p$-reduction of $W$ w.r.t. $\left(E^{*}, E\right)$, then $W_{1}$ is a primitive $p$-contraction of $W$ w.r.t. $\left(E^{*}, E\right)$; (ii) if $W$ is $C_{2} p_{v} W_{0} p_{v}^{-1} C_{1}$ and ( $\exists A)\left[C_{1} C_{2}={ }_{E} A\right.$ and $\left.A \in A(v)\right]$ then $\alpha[B] W_{0}$ where $A \sim B$ is a primitive p-contraction of $W$ w.r.t. $\left(E^{*}, E\right)$; (iii) if $W$ is $C_{2} p_{v}^{-1} W_{0} p_{v} C_{1}$ and $(\exists B)\left[C_{1} C_{2}={ }_{E} B\right.$ and $\left.B \in B(v)\right]$, then $\alpha[A] W_{0}$ where $A \sim B$ is a primitive $p$-contraction of $W$ w.r.t. $\left(E^{*}, E\right)$. If none of the above is applicable to $W$ we say $W$ is $p$-contracted w.r.t. $\left(E^{*}, E\right)$. Always w.r.t. $\left(E^{*}, E\right)$, a $p$-contraction of $W$ is any word obtained from $W$ by a sequence of primitive $p$-contractions. We use $\bar{p}\{W\}$ as a variable for such words. A sequence of successive $p$-contractions must terminate in a $p$-contracted word. If we pick some canonical way of obtaining $p$-contractions, we can define a unique word $p\{W\}$ which is obtained from $W$ by taking $p$-contractions for as long as possible. Then $p\{W\}$ is $p$-contracted (cf. the definition of $p[W]$ in $\S 2$ of [7]).

Let $U$ be any word of $E^{*}$; a cyclic permutation of $U$ is any word $V$ such that there exist words $W_{1}, W_{2}$ such that $U$ is $W_{1} W_{2}$ and $V$ is $W_{2} W_{1}$. The p-projection of $U$ is the word obtained from $U$ by deleting all symbols of $U$ except $p$-symbols; we write $\pi_{p}[U]$ for this. Two words $U, V$ are $p$-parallel if $\pi_{p}[U]$ is $\pi_{p}[V]$ and $p$-circumparallel if $\pi_{p}[V]$ is a cyclic permutation of $\pi_{p}[U]$. Finally we write $l_{p}(U)$ for the number of occurrences of $p$-symbols in $U$.

General Lemma 1. Suppose $\operatorname{Cond}_{J L B}\left(E^{*}, E, p_{v}\right)$ holds and let $U, V$ be p-reduced words of $E^{*}$. If $U={ }_{E^{*}} V$, then $U, V$ are p-parallel. 
Proof. We proceed by induction w.r.t. $l_{p}(U)$. Suppose $l_{p}(U)=0$; now $U^{-1} V=E_{E^{*}} \mathbf{1}$ whence by Britton's Lemma (i.e. Lemma 4 of [9]) $U^{-1} V$ is either $p$-free or not $p$-reduced. The latter is impossible and so $V$ must be $p$-free. Let $l_{p}(U)>0$; again $U^{-1} V={ }_{E^{*}} 1$ and, by Britton's Lemma, $U^{-1} V$ is not $p$-reduced. Since $U^{-1}$ must be $p$-reduced, we have either (1) $\dot{U}$ is $X p_{v} U^{\prime}, V$ is $Y p_{v} V^{\prime}$ where $X, Y$ are $p$ free and $(\exists A)\left[X^{-1} Y={ }_{E} A\right.$ and $\left.A \in A(v)\right]$ or (2) $U$ is $X p_{v}^{-1} U^{\prime}, V$ is $Y p_{v}^{-1} V^{\prime}$ where $X, Y$ are $p$-free and $(\exists B)\left[X^{-1} Y={ }_{E} B\right.$ and $\left.B \in B(v)\right]$.

Case (1). $1={ }_{E^{*}} U^{\prime-1} p_{v}^{-1} X Y p_{v} V^{\prime}={ }_{E^{*}} U^{\prime-1} B V^{\prime}$ where $A \sim B$. Hence $U^{\prime}={ }_{E^{*}} B V^{\prime}$ and the result follows from the hypothesis of induction applied to $U^{\prime}$ and $B V^{\prime}$.

Case (2). The argument is similar.

Gentral Lemma 2. Suppose Cond ${ }_{L B}\left(E^{*}, E, p_{v}\right)$ holds and let $U, V$ be words of $E^{*}$. Then $(\exists W) W^{-1} U W={ }_{E^{*}} V$ if and only if $(\exists W) W^{-1} \bar{p}\{U\} W={ }_{E^{*}} \bar{p}\{V\}$.

Proof. We need consider only a single primitive $p$-contraction of $U$. The result follows by induction since conjugacy is an equivalence relation. If $\bar{p}\{U\}$ is a $p$-reduction of $U$, then $\bar{p}\{U\}={ }_{E^{*}} U$ and the lemma is immediate. So suppose that $U$ is $C_{2} p_{v} U_{\mathbf{0}} p_{v}^{-1} C_{1}$ where $C_{1} C_{2}={ }_{E} A$. Then

$$
\begin{aligned}
1={ }_{E^{*}} W^{-1} U W V^{-1} & ={ }_{E^{*}} W^{-1} C_{1}^{-1} p_{v} p_{v}^{-1} C_{1} C_{2} p_{v} U_{0} p_{v}^{-1} C_{1} W V^{-1} \\
& ={ }_{E^{*}} W^{-1} C_{1}^{-1} p_{v} B U_{0} p_{v}^{-1} C_{1} W V^{-1}
\end{aligned}
$$

where $A \sim B$. Since $B={ }_{E^{*}} \alpha[B]$, the lemma follows. The dual case is similar.

Gentrai Lemma 3. Suppose $\operatorname{Cond}_{J L B}\left(E^{*}, E, p_{v}\right)$ holds and let $U, V$ be p-contracted words of $E^{*}$ not both p-free. If $(\exists W) W^{-1} U W={ }_{E^{*}} V$, then $U, V$ are p-circumparallel and either

(i) there exist words $U_{0}, V_{0}, A$ such that

(a) $U_{0}, V_{0}$ are cyclic permutations of $U, V$ respectively;

(b) $U_{0}, V_{0}$ each have $p_{v}^{-1}$ as final symbol;

(c) $A^{-1} U_{0} A={ }_{E^{*}} V_{0}$; or

(ii) there exist words $U_{0}, V_{0}, B$ such that

(a) $U_{0}, V_{0}$ are cyclic permutations of $U, V$ respectively;

(b) $U_{0}, V_{0}$ each have $p_{v}$ as final symbol;

(c) $B^{-1} U_{0} B=E_{E^{*}} V_{0}$.

Conversely if (i) or (ii) holds, then $(\exists W) W^{-1} U W={ }_{E^{*}} V$.

Proof. We proceed in two stages. Firstly we prove (\#) there exist $U^{*}, V^{*}, X$ such that

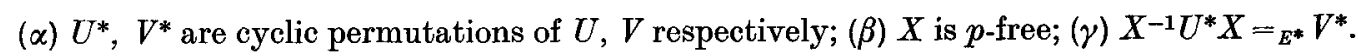


Let us assume $U$ is not $p$-free (there is no loss of generality in doing so). We proceed by induction w.r.t. $l_{p}(W)$. Let $l_{p}(W)=0$; take $X$ to be $W, U^{*}$ to be $U$ and $V^{*}$ to be $V$. Let $l_{p}(W)>0 ;$ since $W^{-1} U W={ }_{E^{*}} V, W^{-1} U W V^{-1}={ }_{E^{*}} 1$. If $W$ is not $p$-reduced, then $\left(\exists W_{1}\right) W={ }_{E^{*}} W_{1}$ and $l_{p}\left(W_{1}\right)<l_{p}(W)$. Then (\#) follows by the induction hypothesis.

So suppose $W$ is $p$-reduced. Since $U, V$ are $p$-contracted, it follows that $U, V^{-1}$ are $p$-reduced. Also $W^{-1}$ must be $p$-reduced. By Britton's Lemma, a $p$-reduction is applicable to $W^{-1} U W V^{-1}$. Three possible cases occur.

(1) $W^{-1}$ is $W_{1}^{-1} p_{v}^{-\varepsilon} Z^{-1}, U$ is $Y p_{v}^{\varepsilon} U_{1}$ with $Y, Z p$-free and $p_{v}^{-\varepsilon} Z^{-1} Y p_{v}^{\varepsilon}$ induces a $p$-reduction.

(2) $U$ is $U_{1} p_{v}^{-\varepsilon} Y, W$ is $Z p_{v}^{e} W_{1}$ with $Y, Z p$-free and $p_{v}^{-\varepsilon} Y Z p_{v}^{\varepsilon}$ induces a $p$-reduction.

(3) $W$ is $W_{1} p_{v}^{-\varepsilon} Z, V^{-1}$ is $Y^{-1} p_{v}^{\varepsilon} V_{1}^{-1}$ with $Y, Z p$-free and $p_{v}^{-\varepsilon} Z Y^{-1} p_{v}^{\varepsilon}$ induces a $p$-reduction.

Case (1). Let $\varepsilon=1$; then $(\exists A) Z^{-1} Y={ }_{E^{*}} A$ whence $(\exists A) Z={ }_{E} Y A^{-1}$. Then

$$
\begin{aligned}
1 & ={ }_{E^{*}} W_{1}^{-1} p_{v}^{-1} Z^{-1} Y p_{v} U_{1} Z p_{v} W_{1} V^{-1}={ }_{E^{*}} W_{1}^{-1} B U_{1} Z p_{v} W_{1} V^{-1} \\
& ={ }_{E^{*}} W_{1}^{-1} B U_{1} Y A^{-1} p_{v} W_{1} V^{-1}={ }_{E^{*}} W_{1}^{-1} B U_{1} Y p_{v} B^{-1} W_{1} V^{-1}
\end{aligned}
$$

where $A \sim B$. Since taking cyclic permutations is an equivalence relation, (\#) follows from the hypothesis of induction since $l_{p}\left(B^{-1} W_{1}\right)=l_{p}(W)-1$. The arguments for $\varepsilon=-1$ and cases (2) and (3) are similar to the above.

Let $U^{*}, V^{*}, X$ be as given by (\#). Since $U$ and $V$ are $p$-contracted, the $U^{*}$ and $V^{*}$ must be $p$-reduced. Since $X^{-1} U^{*} X={ }_{E^{*}} V^{*}$ with $X p$-free it follows that $U^{*}, V^{*}$ are $p$-parallel. Thus $U, V$ are $p$-circumparallel. Let $U^{*}$ be $U_{1} p_{v}^{\varepsilon} Y$ and $V^{*}$ be $V_{1} p_{v}^{\varepsilon} Z$ where $Y, Z$ are $p$-free. By Britton's Lemma ( $\exists A) Y X Z^{-1}={ }_{E} A$ or $(\exists B) Y X Z^{-1}={ }_{E} B$ according as $\varepsilon=\mp 1$. Take $U_{0}$ to be $Y U_{1} p_{v}^{\varepsilon}, V_{0}$ to be $Z V_{1} p_{v}^{\varepsilon}$ and the result follows readily.

Let $U, V$ be two words of $E^{*}$, not $p$-free. We say $V$ is a right (left) conjugate of $U$ by $W$ w.r.t. $\left(E^{*}, E, p\right)$ if $W^{-1} U W={ }_{E^{*}} V$ and there exists a sequence of $p$-reductions of $W^{-1} U W$ terminating in a $p$-reduced word in which the $p$-symbols of $W$ in left to right order (of $W^{-1}$ in right to left order) are the rightmost (leftmost) symbols of the words $p_{v}^{-\varepsilon} C p_{v}^{\varepsilon}$ which induce the successive $p$-reductions.

GENGRAL LEMMA 4. Suppose $\operatorname{Cond}_{J L B}\left(E^{*}, E, p_{v}\right)$ holds. Let $U, V$ be $p$-contracted words of $E^{*}$, neither $p$-free, and $W$ a p-reduced word of $E^{*}$ such that $W^{-1} U W={ }_{E^{*}} V$. Then $V$ is either a right or a left conjugate of $U$ by $W$ w.r.t. $\left(E^{*}, E, p\right)$. If $W$ is not p-free, these cases are mutually exclusive and there is exactly one primitive p-reduction applicable to $W^{-1} U W$. 
Proof. We proceed by induction w.r.t. $l_{p}(W)$. If $l_{p}(W)=0$ the assertion is vacuous. Let $l_{p}(W)>0$.

Case (1). $l_{p}(U)=1$. Let $U$ be $Y_{1} p_{v 1}^{\eta} Y_{2}$ and $W$ be $X p_{v_{1}}^{\varepsilon} W_{1}$ with $Y_{1}, Y_{2}, X p$-free. Then $W^{-1} U W$ is $W_{1}^{-1} p_{v_{a}}^{-\varepsilon} X^{-1} Y_{1} p_{v_{1}}^{\eta} Y_{2} X p_{v_{1}}^{\varepsilon} W_{1}$. It follows from General Lemma 3 (GL 3 ) that $l_{p}(V)=1$ and then from GL 1 that $W^{-1} U W$ is not $p$-reduced. Thus either

(a) $v_{1}=v_{2}, \eta=\varepsilon$ and $(\exists A) X^{-1} Y_{1}={ }_{E} A$ or $(\exists B) X^{-1} Y_{1}={ }_{E} B$ according as $\varepsilon= \pm 1$; or

(b) $v_{1}=v_{2}$ and $\eta=-\varepsilon$ and $(\exists A) Y_{2} X={ }_{E} A$ or $(\exists B) Y_{2} X={ }_{E} B$ according as $\varepsilon= \pm 1$.

These possibilities are mutually exclusive.

(a) Consider $\varepsilon=1$; then $W_{1}^{-1} \alpha[B] Y_{2} X p_{v} W_{1}=E_{E^{*}} V$. Now $\alpha[B] Y_{2} X p_{v}$ is certainly $p$-contracted and so we may apply the inductive hypothesis since $l_{p}\left(W_{1}\right)=l_{p}(W)-1$. Suppose $V$ were a right conjugate of $\alpha[B] Y_{2} X p_{v}$ by $W_{1}$. Then $W$ would not be $p$-reduced. So $V$ must be a left conjugate of $\alpha[B] Y_{2} X p_{v}$ by $W_{1}$. Hence $V$ is a left conjugate of $U$ by $W$. Since (a) and (b) are mutually exclusive, $V$ cannot be a right conjugate of $U$ by $W$. A similar argument holds when $\varepsilon=-1$.

The argument for (b) is dual to that for (a).

Case (2). $l_{p}(U)>1$. Let $U$ be $Y_{1} p_{v_{1}}^{\eta_{1}} U_{0} p_{v_{2}}^{\eta_{3}} Y_{2}$ and $W$ be $X p_{v_{3}}^{e} W_{1}$ with $Y_{1}, Y_{2}$ and $X$ $p$-free. Again by GL 3 and GL $1, W^{-1} U W$ is not $p$-reduced. Four possible cases occur; we shall show that they are mutually exclusive. The cases are

(a) $v_{2}=v_{3}, \varepsilon=1, \eta_{2}=-1,(\exists A) Y_{2} X={ }_{E} A$;

(b) $v_{2}=v_{3}, \varepsilon=-1, \eta_{2}=1,(\exists B) Y_{2} X={ }_{E} B$;

(c) $v_{1}=v_{3}, \varepsilon=1, \eta_{1}=1,(\exists A) X^{-1} Y_{1}={ }_{E} A$;

(d) $v_{1}=v_{3}, \varepsilon=-1, \eta_{1}=1,(\exists B) X^{-1} Y_{1}={ }_{E} B$.

Suppose (a) occurs; then (b) and (d) are immediately impossible. Suppose (c) occurs; let $Y_{2} X={ }_{E} A^{*}$ and $X^{-1} Y_{1}={ }_{E} A^{\sim}$. Then $Y_{2} Y_{1}={ }_{E} A^{*} A^{\sim}$ and also $v_{2}=v_{3}=v_{1}$ and $\eta_{2}=-1$, $\eta_{1}=1$. This means that $U$ is not $p$-contracted which is a contradiction. Using similar arguments we can show (b) and (d) mutually exclusive whence it follows that (a), (b), (c) and (d) are mutually exclusive.

Now we prove the assertion of the lemma. We shall examine only case (a) in detail; the others are similar. In case (a), $W^{-1} U W={ }_{E^{*}} W_{1}^{-1} p_{v_{\mathrm{s}}}^{-1} X^{-1} Y_{1} p_{v_{1}}^{\eta_{1}} U_{0} \alpha[B] W_{1}$ where $A \sim B$. Writing $U^{*}$ for $p_{v_{1}}^{-1} X^{-1} Y_{1} p_{v_{1}}^{\eta_{1}} U_{0} \alpha[B]$, we can easily show that the assumption that $U^{*}$ is not $p$-contracted contradicts the fact that $U$ is $p$-contracted. The inductive hypothesis may therefore be applied to $U^{*}$ and $V$. Since $W$ is $p$-reduced, $W_{1}^{-1} p_{v_{z}}^{-1}$ must be $p$-reduced 
so that $V$ must be a right conjugate of $U^{*}$ by $W_{1}$. Hence $V$ must be a right conjugate of $U$ by $W$. Since (a), (c) and (d) are mutually exclusive $V$ cannot be a left conjugate of $U$.

General Lemma 5. Suppose $\operatorname{Cond}_{J L B}\left(E^{*}, E, p_{v}\right)$ holds. Let $U$ and $V$ be p-contracted words of $E^{*}$, neither $p$-free, and $W$ a p-reduced word of $E^{*}$. If $V$ is a left conjugate of $U$ w.r.t. $\left(E^{*}, E, p\right)$ by $W$, then $U$ is a right conjugate of $V$ w.r.t. $\left(E^{*}, E, p\right)$ by $W^{-1}$.

Proof. Since $W^{-1} U W={ }_{E^{*}} V, U={ }_{E^{*}} W V W^{-1}$. By GL $4 U$ is either a left conjugate or a right conjugate of $V$ by $W^{-1}$. If $W$ is $p$-free, the lemma is trivial. If $W$ is not $p$-free, then exactly one $p$-reduction is applicable to $W V W^{-1}$. We want to show that it must "straddle" $V W^{-1}$. Suppose not; then it must straddle $W V$. Now $W V W^{-1} U^{-1}={ }_{E^{*}} 1$ whence $p\left[W V W^{-1} U^{-1}\right]$ must be $p$-free. Since $V$ is a left conjugate of $U$ by $W, U W$ and hence $W^{-1} U^{-1}$ are $p$-reduced. Thus there is exactly one $p$-reduction applicable to $W V W^{-1} U^{-1}$ and it straddles $W V$. Hence there is exactly one possible sequence of $p$-reductions from $W V W^{-1} U^{-1}$. But since $l_{p}\left(V W^{-1} U^{-1}\right)>l_{p}(W)$, this sequence will not terminate in a $p$-free word. This is the necessary contradiction.

General Lemma 6. Suppose $\operatorname{Cond}_{J L B}\left(E^{*}, E, p_{v}\right)$ holds and let $U$ be $U_{1} p_{v}$ and $V$ be $V_{1} p_{v}$. Then $(\exists B \in B(v)) B^{-1} U B={ }_{E^{*}} V$ if and only if $(\exists A \in A(v)) A^{-1} U_{1}^{-1} p_{v}^{-1} A={ }_{E^{*}} V_{1}^{-1} p_{v}^{-1}$.

Proof. If $B^{-1} U B={ }_{E^{*}} V$, then $B^{-1} U_{1} A p_{v}={ }_{E^{*}} V_{1} p_{v}$ where $A \sim B$. Hence $p_{v} B^{-1} U_{1} A={ }_{E^{*}}$ $p_{v} V_{1}$ implying that $A^{-1} p_{v} U_{1} A={ }_{E^{*}} p_{v} V_{1}$. Taking inverses gives $A U_{1}^{-1} p_{v}^{-1} A^{-1}={ }_{E^{*}} V_{1}^{-1} p_{v}^{-1}$. The converse is obtained by reversing the above argument.

Generat Lemma 7. Suppose $\operatorname{Cond}_{J L B}\left(E^{*}, E, p_{v}\right)$ holds. Then "(? $U, U$ a word of $\left.E^{*}\right)$ To compute $p\{U\}$ " reduces to (? $X, X$ a word of $E)(\exists A \in A(v)) X={ }_{E} A$ and (? $X, X$ a word of $\left.E\right)$ $(\exists B \in B(v)) X={ }_{E} B$.

Proof. This follows from the definition of $p\{W\}$.

\section{Part II. The word problem}

Let $G_{0}$ be the group presented in the Technical Result.

Let $G_{2}=\left(s_{b}, q, x, r_{i} ; x s_{b}=s_{b} x^{2}, r_{i} s_{b}=s_{b} x r_{i} x, r_{i} \bar{F}_{i} q=q K_{i} r_{i}\right)$

$$
\begin{aligned}
& G_{3}=\left(s_{b}, x, r_{i} ; x s_{b}=s_{b} x^{2}, r_{i} s_{b}=s_{b} x r_{i} x\right) \\
& G_{4}=\left(s_{b}, x ; x s_{b}=s_{b} x^{2}\right)
\end{aligned}
$$


$F^{\prime}(x)=(x ; \varnothing)$, i.e. the free group on $x$. Our $G_{3}$ and $G_{4}$ coincide with $G_{3}^{R}$ and $G_{4}^{R}$ of [9]. Our $G_{0}$ and $G_{2}$ are very similar to but not identical with the $G_{1}$ and $G_{2}$ of [9] (with $N=0$ ). In [7], Boone shows that the $G_{1}$ of [9] has a solvable word problem. In solving the word problem for $G_{0}$, we do little more than paraphrase Boone's argument.

We adopt the following notation.

\begin{tabular}{cl} 
Variable & \multicolumn{1}{c}{ Range } \\
$P$ & $k$-free, $t$-free words \\
$F, G, H$ & $k$-free, $t$-free, $q$-free words \\
$N$ & $k$-free, $t$-free, $q$-free, $r$-free words \\
$R$ & products of $r_{i}, x, i=1,2, \ldots, P$ \\
$A$ & products of $r_{i} \bar{F}_{i}, i=1,2, \ldots, P$ \\
$B$ & products of $K_{i} r_{i}, i=1,2, \ldots, P$ \\
$Q_{\varepsilon}$ & products of $s_{b} x^{\varepsilon}, \varepsilon= \pm 1, b=1,2, \ldots, M$ \\
$J$ & words on $x$ \\
$T_{\varepsilon}$ & words on $x^{(3-\varepsilon) / 2}, \varepsilon= \pm 1$
\end{tabular}

Also we write $A_{i}$ for $r_{i} \bar{F}_{i}$ and $B_{i}$ for $K_{i} r_{i}$ and an arrow " $\longrightarrow$ " for "is reducible to". The above list accords reasonably well with the notation of $\S 1$ of [7].

Lем М А $1 . F(x)$ has a solvable word problem.

Proof. $F(x)$ is a free group.

Lemma 2. $\operatorname{Cond}_{J L B}\left(G_{4}, F^{\prime}(x), s_{b}\right)$ holds.

Proof. Trivial.

Lemma 3. For any $N, s[N]$ is recursively computable.

Proof. The problems (? $(J)\left(\exists T_{\varepsilon}\right) J=T_{\varepsilon}$ in $F^{\prime}(x), \varepsilon= \pm 1$ are solvable.

LEMмA 4 . The word problem for $G_{4}$ is solvable.

Proof. Given $N$, compute $s[N]$. If $s[N]$ is not $s$-free, it follows from GL 1 that $N \neq \mathbf{I}$ in $G_{4}$. If $s[N]$ is $s$-free, $s[N]=1$ in $G_{4}$ if and only if $s[N]=1$ in $F(x)$. Now use Lemma 1.

LamMA 5. $(? N)\left(\exists Q_{\varepsilon}\right) N={ }_{G_{4}} Q_{\varepsilon}$ is solvable.

Proof. Let $N$ be given and let $Q_{\varepsilon}^{*}$ be $s$-parallel to $s[N]$. Then we shall show that $\left(\exists Q_{\varepsilon}\right) N={ }_{G 4} Q_{\varepsilon}$ if and only if $s[N]={ }_{G_{t}} Q_{\varepsilon}^{*}$. This suffices since $s[N]$ is recursively computable. If $s[N]={ }_{G_{4}} Q_{\varepsilon}^{*}$, then $N={ }_{G_{4}} Q_{\varepsilon}^{*}$. 
Conversely suppose $N={ }_{G_{t}} Q_{\varepsilon}$; then $s[N]={ }_{G_{t}} \alpha\left[Q_{\varepsilon}\right]$. But it is easy to see that $\alpha\left[Q_{\varepsilon}\right]$ must be $s$-reduced. Hence $\alpha\left[Q_{\varepsilon}\right]$ is $Q_{\varepsilon}^{*}$.

Lemма 6. $\operatorname{Cond}_{J L B}\left(G_{3}, G_{4}, r_{i}\right)$ holds.

Proof. $\left\{s_{b} x\right\}$ and $\left\{s_{b} x^{-1}\right\}$ are sets of free generators in $G_{4}$.

Lемма 7. The word problem for $G_{3}$ is solvable.

Proof. It follows from Lemma 5 that for any $F, r[F]$ is recursively computable. The word problem for $G_{4}$ is solvable.

Lемма 8. $\left(? F^{\prime}\right)(\exists A) F={ }_{G s} A$ and $(? F)(\exists B) F={ }_{G s} B$ are solvable.

Proof. For any $A$ or $B, \alpha[A]$ or $\alpha[B]$ is $r$-reduced w.r.t. $\left(G_{3}, G_{4}\right)$. If $F$ is given, it then follows that $(\exists A) F={ }_{G_{3}} A$ if and only if $r[F]={ }_{G_{3}} A^{*}$ where $A^{*}$ is $r$-parallel to $r[F]$. But $r\left[F^{\prime}\right]$ is recursively computable and the word problem for $G_{3}$ is solvable.

LEMMA 9. $\operatorname{Cond}_{J L B}\left(G_{2}, G_{3}, q\right)$ holds.

Proof. We show $\left\{A_{i}\right\}$ and $\left\{B_{i}\right\}$ are sets of free generators in $G_{3}$. Let $\prod A_{i j}^{e_{j}}=1$; putting $x=s_{b}=1$ we obtain $\prod r_{i j}^{e j}=1$ in $F\left(r_{i}\right)$ whence it follows that $\left\{A_{i}\right\}$ is a set of free generators. A similar argument holds for $\left\{B_{i}\right\}$.

LEMMA 10. The word problem for $G_{2}$ is solvable.

Proof. For any $P, q[P]$ is recursively computable (by Lemma 8 ). The word problem for $G_{3}$ is solvable.

LEMMA 11. $(? P)(\exists R) P={ }_{G \mathbf{a}} R$ is solvable.

Proof. Let $P$ be given; compute $q[P]$. If $q[P]$ is not $q$-free, then $(\exists R) P={ }_{G_{2}} R$ fails. So suppose that $q[P]$ is $q$-free. It is not hard to verify that $\operatorname{Cond}_{J L B}\left(G_{3}, F\left(x, r_{i}\right), s_{b}\right)$ holds. Moreover it is easy to see that for any $F, s[F]$ is recursively computable (s-reduction w.r.t. $\left.\left(G_{3}, F\left(x, r_{i}\right), s_{b}\right)\right)$. Since $(\exists R) P={ }_{G_{2}} R$ if and only if $s[q[P]]$ is a word on $x$ and $r_{i}$, the lemma follows.

LmMMA 12. $\operatorname{Cond}_{J L B}\left(G_{0}, G_{2},\{t, k\}\right)$ holds.

Proof. Trivial.

Solution 1. The word problem for $G_{0}$ is solvable.

Proof. For any $W, t k[W]$ is recursively computable. The word problem for $G_{2}$ is solvable. 


\section{Part III. The conjugacy problem}

The argument given in this section is very similar to that of Britton in [9]. In particular, Lemmas 14,15 and 16 correspond to Lemmas 6,7 and 8 of Britton.

LемMA 13. (i) Let $S$ be a positive word on the symbols $s_{1}, s_{2}, \ldots, s_{M}$. Then for $\varepsilon= \pm 1$, $(\exists R) r_{i}^{\varepsilon} S={ }_{G_{0}} S R$ and $(\exists R) x^{\varepsilon} S={ }_{G_{0}} S R$.

(ii) Let $S$ be a negative word on the symbols $s_{1}, s_{2}, \ldots, s_{M}$. Then for $\varepsilon= \pm 1,(\exists R) S r_{i}^{e}={ }_{G_{0}} R S$ and $(\exists R) S x^{e}={ }_{G_{0}} R S$.

Proof. This is proved by induction w.r.t. $l(S)$ using the equations $x^{\varepsilon} s_{b}=s_{b} x^{2 \varepsilon}$, $r_{i}^{\varepsilon} s_{b}=s_{b} x^{\varepsilon} r_{i}^{\varepsilon} x^{\varepsilon}, s_{b}^{-1} x^{\varepsilon}=x^{2 \varepsilon} s_{b}^{-1}$ and $s_{b}^{-1} r_{i}^{\varepsilon}=x^{\varepsilon} r_{i}^{\varepsilon} x^{\varepsilon} s_{b}^{-1}, \varepsilon= \pm 1$.

We use capital Greek letters, e.g. $\Delta$, as variables for positive words on the $s$-symbols. Given any $\Delta$, the word $\bar{\Delta}$ is obtained from $\Delta$ by replacing each $s_{b}$ by $s_{b}^{-1}$ (this is consistent with the definition of $\bar{F}_{i}$ in the presentation of $G_{0}$ ).

L.ммм 14. Let $\Delta q \Pi, \Omega q \Phi$ be words of $\mathfrak{I}_{*}$ such that $\Delta q \Pi=\Omega q \Phi$ in $\mathfrak{I}_{*}$. Then $t^{-1} \Pi^{-1} q^{-1} \bar{\Delta}^{-1} t \bar{\Delta} q \Pi$ is conjugate to $t^{-1} \Phi^{-1} q^{-1} \bar{\Omega}^{-1} t \bar{\Omega} q \Phi$ in $G_{0}$.

Proof. Let $W_{1} \rightarrow W_{2} \rightarrow \ldots \rightarrow W_{n}$ where $W_{1}$ is $\Delta q \Pi$ and $W_{n}$ is $\Omega q \Phi$ be the proof that $\Delta q \Pi=\Omega q \Phi$ in $\mathfrak{I}_{*}$. We show firstly, by induction w.r.t. $n$, that $(\exists R)\left(\exists R_{1}\right) \bar{\Delta} q \Pi={ }_{c_{0}} R_{1} \bar{\Omega} q \Phi R$. If $n=1$, this is trivial. So suppose that $n>1$; from the form of the rules of $\mathfrak{I}_{*}$, it follows that every $W_{k}$ must have the form $\Delta_{k} q \Pi_{k}$. In particular $W_{n-1}$ is $\Delta_{n-1} q \Pi_{n-1}$ and either $\Delta_{n-1}$ is $\Omega F_{i}$ or $\Pi_{n-1}$ is $K_{i} \Phi$ according as $F_{i} q \rightarrow q K_{i}$ or $q K_{i} \rightarrow F_{i} q$ is the final rule applied. Assume it is the former; then $\bar{\Delta}_{n-1} q \Pi_{n-1}$ is $\bar{\Omega} \bar{F}_{i} q \Pi_{n-1}={ }_{G_{0}} \bar{\Omega} r_{i}^{-1} q K_{i} r_{i} \Pi_{n-1}$. Since $\bar{\Omega}$ is a negative word and $\Pi_{n-1}$ is a positive word, it follows from Lemma 13 that

$$
\left(\exists R_{2}\right)\left(\exists R_{3}\right) \bar{\Delta}_{n-1} q \Pi_{n-1}={ }_{G_{0}} R_{2} \bar{\Omega}_{q} \Phi R_{3} .
$$

By the inductive assumption we have $\left(\exists R_{4}\right)\left(\exists R_{5}\right) \bar{\Delta} q \Pi={ }_{G_{0}} R_{4} \bar{\Delta}_{n-1} q \Pi_{n-1} R_{5}$. Let $R_{1}$ be $R_{4} R_{2}$ and $R$ be $R_{3} R_{5}$; then $\bar{\Delta} q \Pi=R_{1} \bar{\Omega} q \Phi R$. If the final rule is $q K_{i} \rightarrow F_{i} q$, a similar argument applies.

The lemma now follows easily; for

$$
t^{-1} \Pi^{-1} q^{-1} \bar{\Delta}^{-1} t \bar{\Delta} q \Pi={ }_{G_{0}} t^{-1} R^{-1} \Phi^{-1} q^{-1} \bar{\Omega}^{-1} R_{1}^{-1} t R_{1} \bar{\Omega} q \Phi R={ }_{G_{0}} R^{-1} t^{-1} \Phi^{-1} q^{-1} \overline{\Omega^{-1}} t \bar{\Omega} q \Phi R
$$

because the equalities $t r_{i}^{\varepsilon}=r_{i}^{\varepsilon} t$ and $t x^{\varepsilon}=x^{\varepsilon} t$ hold in $G_{0}$.

LEMMA 15. If $t^{-1} \Pi^{-1} q^{-1} \bar{\Delta}^{-1} t \bar{\Delta} q$ and $t^{-1} \Phi^{-1} q^{-1} \overline{\Omega^{-1}} t \bar{\Omega} q \Phi$ are conjugate in $G_{0}$, then $\left(\exists R_{1}\right)\left(\exists R_{2}\right)(\exists A)(\exists B)\left[\bar{\Omega}^{-1} R_{1} \bar{\Delta} A={ }_{G_{3}} 1, \Phi R_{2} \Pi^{-1} B={ }_{G_{3}} 1\right.$ and $\left.A \sim B\right]$.

Proof. We wish to apply GL 3 taking $t^{-1} \Pi^{-1} q^{-1} \bar{\Delta}^{-1} t \bar{\Delta} q \Pi$ as $U$ and $t^{-1} \Phi^{-1} q^{-1} \bar{\Omega}^{-1} t \bar{\Omega} q \Phi$ as $V$ and $\{t, k\}$ as stable letters. We must show that $U$ and $V$ are $t k$-contracted. Suppose 9-692905 Acta mathematica 122. Imprimé le 21 mars 1969 
$U$ is not $t k$-contracted; there appear to be two ways in which this could occur. But in either case we should obtain the conclusion that $(\exists R) \Delta q \Pi={ }_{G} R$. Since $\operatorname{Cond}_{J L B}\left(G_{2}, G_{3}, q\right)$ holds, by GL 1 this is impossible. In a similar way, $V$ can be shown to be $t k$-contracted.

Now we apply GL 3 ; we must have either

(i) $\quad(\exists R) R^{-1} \Pi^{-1} q^{-1} \bar{\Delta}^{-1} t \bar{\Delta} q \Pi t^{-1} R={ }_{G_{0}} \Phi^{-1} q^{-1} \bar{\Omega}^{-1} t \bar{\Omega} q \Phi t^{-1}$ or

(ii) $\quad(\exists R) R^{-1} \bar{\Delta} q \Pi t^{-1} \Pi^{-1} q^{-1} \bar{\Delta}^{-1} t R={ }_{G o} \bar{\Omega} q \Phi t^{-1} \Phi^{-1} q^{-1} \bar{\Omega}^{-1} t$.

We shall examine (i); (ii) is similar. Since

$$
t^{-1} R={ }_{G_{0}} R t^{-1}, \quad R^{-1} \Pi^{-1} q^{-1} \bar{\Delta}^{-1} t \bar{\Delta} q \Pi R={ }_{G_{0}} \Phi^{-1} q^{-1} \bar{\Delta}^{-1} t \bar{\Omega} q \Phi .
$$

By Britton's Lemma, we have $\left(\exists R^{\prime}\right) \Delta q \Pi R \Phi^{-1} q^{-1} \bar{\Omega}^{-1}={ }_{G_{2}} R^{\prime}$. Using $q$ as stable letter w.r.t. $\left(G_{2}, G_{3}\right)$, we obtain $(\exists B) \Pi R \Phi^{-1}={ }_{G_{3}} B$. Upon substitution this produces $\bar{\Delta} q B q^{-1} \bar{\Omega}^{-1}={ }_{G s} R^{\prime}$. It follows from the defining relations $r_{i} \bar{F}_{i} q=q K_{i} r_{i}$ that $q B q^{-1}={ }_{G_{3}} A$ where $A \sim B$. Hence $\bar{\Delta} A \bar{\Omega}^{-1}={ }_{G_{2}} R^{\prime}$ and by Lemma 3 of [9], this equality holds in $G_{3}$. Thus $\bar{\Omega}^{-1} R^{\prime-1} \Delta A={ }_{G_{3}} 1$ and from a previous equation we obtain $\Phi R^{-1} \Pi^{-1} B={ }_{G_{3}} 1$. Take $R_{1}$ to be $R^{\prime-1}$ and $R_{2}$ to be $R^{-1}$.

LеммA 16. If $\left(\exists R_{1}\right)\left(\exists R_{2}\right)(\exists A)(\exists B)\left[\bar{\Omega}^{-1} R_{1} \Delta A={ }_{G_{3}} 1, \Phi R_{2} \Pi^{-1} B={ }_{G_{s}} 1\right.$ and $\left.A \sim B\right]$, then $\Delta q \Pi=\mathfrak{I}_{*} \Omega q \Phi$.

Proof. We proceed by induction w.r.t. the number of $r$-symbols in $A$. Let $l_{r}(A)=0$; then $\bar{\Omega}^{-1} R_{1} \bar{\Delta}={ }_{G 3} 1$ and $\Phi R_{2} \Pi^{-1}={ }_{G s} 1$. Putting $r_{i}=x=1$, we obtain $\bar{\Omega}^{-1} \bar{\Delta}=1$ in $F\left(s_{b}\right)$ and $\Phi \Pi^{-1}=1$ in $F\left(s_{b}\right)$. Thus $\bar{\Omega}=\bar{\Delta}$ in $F\left(s_{b}\right)$ and $\Phi=\Pi$ in $F\left(s_{b}\right)$. This means $\Omega$ is $\Delta$ and $\Phi$ is $\Pi$. Let $l_{r}(A)>0$; if $A, B$ are not $\alpha$-reduced, then $\bar{\Omega}^{-1} R_{1} \bar{\Delta} \alpha[A]={ }_{G_{s}} 1$ and $\Phi R_{2} \Pi^{-1} \alpha[B]={ }_{G_{s}} 1$ and $\alpha[A] \sim \alpha[B]$. The result follows by the inductive hypothesis.

So suppose $A$ and $B$ are $\alpha$-reduced; we can $\alpha$-reduce $R_{1}$ and $R_{2}$ to obtain $\bar{\Omega}^{-1} \alpha\left[R_{1}\right] \bar{\Delta} A={ }_{G_{s}} 1$ and $\Phi \propto\left[R_{2}\right] \Pi^{-1} B={ }_{G s} 1$. We write $A$ as $A_{i}^{\varepsilon} A^{\prime}$ and consider the case when $\varepsilon=-1$. Now $\alpha\left[R_{1}\right]$ and $A$ are $r$-reduced w.r.t. $\left(G_{3}, G_{4}\right)$, but $A$ is not $r$-free so an $r$ reduction must apply to $\bar{\Omega}^{-1} \alpha\left[R_{1}\right] \Delta A$. This means that $\bar{\Omega}^{-1} \alpha\left[R_{1}\right] \bar{\Delta} A$ is $\bar{\Omega}^{-1} R^{*} r_{i} x^{m} \bar{\Delta} \bar{F}_{i}^{-1} r_{i}^{-1} A^{\prime}$ and $\left(\exists Q_{-1}\right) x^{m} \bar{\Delta} \bar{F}_{1}^{-1}={ }_{G_{4}} Q_{-1}$-and we may assume $Q_{-1}$ is $\alpha$-reduced. Then $x^{m} \bar{\Delta}={ }_{G_{4}} Q_{-1} \bar{F}_{i}$; we claim that $\bar{\Delta}$ and $Q_{-1} \bar{F}_{i}$ are both $s$-reduced w.r.t. $\left(G_{4}, F(x)\right)$.

Certainly $\bar{\Delta}$ is $s$-reduced; since $Q_{-1}$ is $\alpha$-reduced it is $s$-reduced, so an $s$-reduction in $Q_{-1} \bar{F}_{i}$ must "straddle" $Q_{-1}$ and $\bar{F}_{i}$. But this means that $Q_{-1} \bar{F}_{i}$ is $Q_{-1}^{*} s_{b} x^{-1} s_{b}^{-1} \bar{\Theta}$ and $x^{-1}$ is a word on $x^{2}$. This is a contradiction. By GL $1, x^{m} \bar{\Delta}$ and $Q_{-1} \bar{F}_{i}$ must be $s$-parallel whence $\Delta$ is $\Delta_{2} F_{i}$. Then $\bar{\Omega}^{-1} \alpha\left[R_{1}\right] \bar{\Delta}_{2} \bar{F}_{i} \bar{F}_{i}^{-1} r_{i}^{-1} A^{\prime}={ }_{G_{9}} 1$ whence, for some $R^{\sim}, \bar{\Omega}^{-1} \propto\left[R_{1}\right] R-\bar{\Delta}_{2} A^{\prime}={ }_{G_{3}} 1$. Also $\Phi \propto\left[R_{2}\right] \Pi^{-1} r_{i}^{-1} K_{i}^{-1} B^{\prime}={ }_{G s} 1$ whence, for some $R^{\prime}, \Phi \propto\left[R_{2}\right] R^{\prime} \Pi^{-1} K_{i}^{-1} B^{\prime}={ }_{G_{3}} 1$. By the inductive hypothesis, $\Delta_{2} q K_{i} \Pi=\mathfrak{I}_{*} \Omega q \Phi$. But $\Delta q \Pi$ is $\Delta_{2} F_{i} q \Pi=\mathfrak{I}_{*} \Delta_{2} q K_{i} \Pi$.

REDUCTION I. $(? \Delta q \Pi, \Omega q \Phi) \Delta q \Pi=\tau_{*} \Omega q \Phi \longrightarrow(? U, V)(\exists W) W^{-1} U W={ }_{G_{0}} V$.

Proof. This is immediate by Lemmas 14, 15 and 16. 


\section{Part IV. The degree}

$\S 1$. In Parts II and III we have viewed $G_{0}$ as a tower of presentations, viz.

$G_{0}$
$\mid t, k$
$G_{2}$
$\mid q$
$G_{3}$
$\mid r_{\imath}$
$G_{4}$
$\mid s_{\eta}$
$F(x)$,

where each presentation $E$ is a subpresentation of that immediately above it with the indicated letters as stable letters. Moreover, the isomorphism condition holds for each pair, so that we actually have a tower of groups. Our line of argument will be to decompose $G_{0}$ in various ways to obtain different towers of presentations and we shall show that these actually give towers of groups. Let

$$
\begin{aligned}
& H=\left(s_{b}, x, t, k, r_{i} ; x s_{b}=s_{b} x^{2}, x t=t x, k x=x k, r_{i} s_{b}=s_{b} x r_{i} x, r_{i} t=t r_{i}, r_{i} k=k r_{i}\right) \\
& H_{1}=\left(s_{b}, x, t, k, q ; x s_{b}=s_{b} x^{2}, x t=t x, k x=x k\right) \\
& H_{2}=\left(s_{b}, x, t, k ; x s_{b}=s_{b} x^{2}, x t=t x, k x=x k\right) \\
& H_{3}=\left(s_{b}, x, q ; x s_{b}=s_{b} x^{2}\right)
\end{aligned}
$$

Notice that, strictly according to Britton's definition, $q$ is not a stable letter for $H_{1}$ with basis $H_{2}$ since $H_{1}$ has no relations involving $q$. But by regarding $H_{1}$ as $H_{2} *$ [q], the free product of $\mathrm{H}_{2}$ and the infinite cyclic group on $q$, we can regard Lemmas 3 and 4 of [9] as applicable to $\left(H_{1}, H_{2}, q\right)$ with $A(v)=B(v)=$ the group generated by the identity element. $\left({ }^{1}\right)$ Similar remarks apply to $\left(H_{3}, G_{4}, q\right)$.

With this in mind we obtain Diagram $A$ which is to be interpreted as follows. If $E^{*}$ is connected to $E$ by a descending line marked $p$, then $\left\{p_{v}\right\}$ is a set of stable letters for $E^{*}$ with basis $E$. We shall show that $\operatorname{Cond}_{J L B}\left(E^{*}, E, p_{v}\right)$ holds for all such pairs on the diagram.

We extend our list of notation.

(1) The two lemmas of Britton are intimately connected with the theory of free products with amalgamated subgroups. The above is an illustration of this for a very special case. 

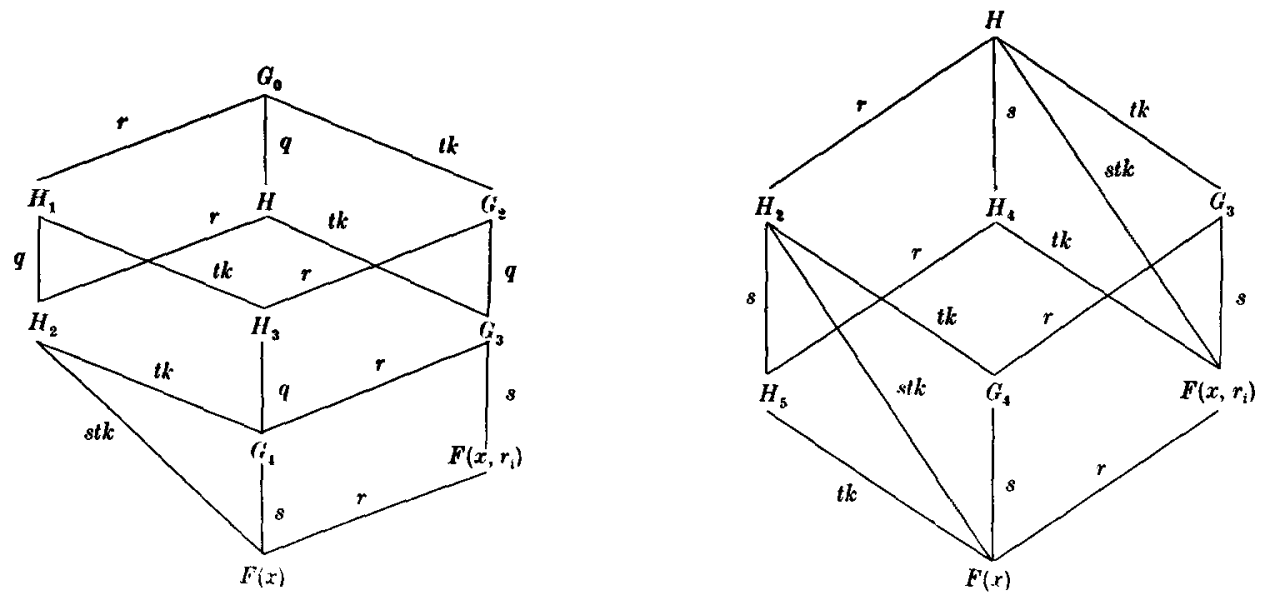

Diagram A

Variable

Range

$U, V, W \quad$ any word

$D, E \quad r$-free words

$X, Y, Z \quad q$-free words

$L, M \quad r$-free, $q$-free words

$C_{\varepsilon} \quad$ products of $s_{b} x^{\varepsilon}, t, k$

$P_{1, i} \quad$ products of $s_{b} x, t, k, q K_{i}$

$P_{-1, i} \quad$ products of $s_{b} x^{-1}, t, k, \bar{F}_{i} q$

$S_{+1} \quad$ products of $x, r_{i}$

$S_{-1} \quad$ products of $x^{2}, x r_{i} x$

Also let $l(W)=$ total number of symbols in $W$ (i.e. symbol occurrences in $W$ ).

§ 2. Lемма 17. (i) $\operatorname{Cond}_{J L B}\left(H_{2}, F(x),\left\{s_{b}, t, k\right\}\right)$ holds. (ii) $\operatorname{Cond}_{J L B}\left(H_{1}, H_{2}, q\right)$ holds. (iii) $\operatorname{Cond}_{J L B}\left(G_{0}, H_{1}, r_{i}\right)$ holds.

Proof. (i) To prove this we must show that $x \rightarrow x^{2}$ generates an isomorphism in $F^{\prime}(x)$ and also that $x \rightarrow x$ generates an isomorphism in $F(x)$. But these are both trivial.

Notice that we do not verify Britton's strong isomorphism condition; indeed the strong isomorphism condition clearly fails.

(ii) We have already remarked how we can assume this.

(iii) Again we verify only the isomorphism condition and not the strong isomorphism condition. We show that for each $i$, the sets $\left\{\bar{F}_{i} q, s_{b} x^{-1}, t, k\right\}$ and $\left\{q K_{i}, s_{b} x, t, k\right\}$ are sets of free generators in $H_{1}$.

Suppose $\prod \gamma_{k}^{e_{k}}={ }_{H_{1}} 1$, where $\gamma$ is $s_{b} x^{-1}, t, k$ or $\bar{F}_{i} q$ (with $i$ fixed). 
Case (a). $\prod \gamma_{k}^{e_{k}}$ is $q$-free. Then $\prod \gamma_{k}^{e_{k}}={ }_{H_{2}} 1$ since (ii) holds. If $\prod \gamma_{k}^{e_{k}}$ is non-empty it must contain either an $s_{b}$, a $k$ or a $t$. Thus it contains $s_{b}^{-\varepsilon} C s_{b}^{\varepsilon}$ where $\left(\exists T_{\varepsilon}\right) C=T_{\varepsilon}$ in $F(x)$ or $t^{-\varepsilon} C t^{\varepsilon}$ or $k^{-\varepsilon} C k^{\varepsilon}$ where $(\exists J) C=J$ in $F(x)$. In either case, $\prod \gamma_{k}^{e_{k}}$ cannot be $\alpha$-reduced.

Case (b). $\prod \gamma_{k}^{e_{k}}$ is not $q$-free. Then $\prod \gamma_{k}^{e_{k}}$ must contain as a subword either $\bar{F}_{i} q \gamma_{m}^{e_{m}} \ldots \gamma_{n}^{e_{n}} q^{-1} \bar{F}_{i}^{-1}$ with $\gamma_{m}^{e_{m}} \ldots \gamma_{n}^{e_{n}}={ }_{H_{2}} 1$ or $q^{-1} \bar{F}_{i}^{-1} \gamma_{m}^{e_{m}} \ldots \gamma_{n}^{e_{n}} \bar{F}_{i} q$ with $\bar{F}_{i}^{-1} \gamma_{m}^{e_{m}} \ldots \gamma_{n}^{e_{n}} \bar{F}_{i}={ }_{H_{2}} 1$. Write $M$ for $\gamma_{m}^{e_{m}} \ldots \gamma_{n}^{e_{n}}$; in both cases $M={ }_{H_{2}} 1$. If $M$ is empty, then it is immediate that $\prod \gamma_{k}^{e_{k}}$ is not $\alpha$-reduced. If $M$ is non-empty, then, by the argument of (a), $M$ is not $\alpha$-reduced whence $\prod \gamma_{k}^{e k}$ is not $\alpha$-reduced.

The argument for $\left\{s_{b} x, t, k, q K_{i}\right\}$ is virtually identical.

SoLution 2. The word problem for $\mathrm{H}_{2}$ is solvable.

Proof. $L={ }_{H_{2}} \mathrm{l}$ if and only if $L={ }_{G_{0}} 1$ and $G_{0}$ has solvable word problem.

SoLUtion 3. For any $L$, stk $[L]$ is recursively computable (stk-reduction w.r.t. $\left(H_{2}, F(x)\right)$ ).

Proof. By Lemma 1.1 of [7], we need to be able to solve (?J) $\left(\exists T_{\varepsilon}\right) J=T_{\varepsilon}$ in $F^{\prime}(x)$ and $(? J)$ "Is $J$ a word on $x$ ". But these are both solvable.

Soldtion 4. $(? L)\left(\exists C_{\varepsilon}\right) L={ }_{H_{2}} C_{\varepsilon}$ is solvable.

Proof. $\left(\exists C_{\varepsilon}\right) L={ }_{H_{2}} C_{\varepsilon}$ if and only if $s t k[L]={ }_{H_{2}} C_{\varepsilon}^{*}$ where $C_{\varepsilon}^{*}$ is $s t k$-parallel to $s t k[L]$. The result follows since $s t k[L]$ is recursively computable and $H_{2}$ has a solvable word problem.

Soldtion 5. For any $D, q[D]$ is recursively computable (q-reduction w.r.t. $\left(H_{1}, H_{2}\right)$ ).

Proof. We need to solve $(? L) L={ }_{H_{2}} 1$; but this is solvable.

Solution 6. (?D) $\left(\exists P_{\varepsilon, i}\right) D={ }_{H_{1}} P_{\varepsilon, i} \varepsilon= \pm 1$ is solvable.

Proof. The argument for $\varepsilon=1$ is dual to that for $\varepsilon=-1$. Now $\left(\exists P_{-1, i}\right) D={ }_{H_{2}} P_{-1, i}$ if and only if $\left(\exists P_{-1, i}\right) q[D]={ }_{H_{1}} P_{-1, i}$. So it suffices to consider $D q$-reduced w.r.t. $\left(H_{1}, H_{2}\right)$.

We proceed by induction w.r.t. $l_{q}(D)$. Let $l_{q}(D)=0$; then $D={ }_{H_{1}} P_{-1, i}$ if and only if $D={ }_{H_{1}} \alpha\left[P_{-1, i}\right]$. Since $\alpha\left[P_{-1, i}\right]$ is $q$-reduced, it must be $q$-free. Thus $\left(\exists P_{-1, i}\right) D={ }_{H_{1}} P_{-1, i}$ if and only if $\left(\exists C_{-1}\right) D={ }_{H_{1}} C_{-1}$. The result follows by Lemma 3 of [9] and Solution 4. Let $l_{q}(D)>0$; write $D$ as $D^{\prime} q^{\eta} L$ where $L$ is $q$-free. The argument for $\eta=-1$ is dual to the argument for $\eta=1$. So suppose $\eta=1$; by the inductive hypothesis and Solution 4, we can determine whether or not (i) $\left(\exists C_{-1}\right) L={ }_{H_{3}} C_{-1}$ and (ii) $\left(\exists P_{-1, i}\right) D^{\prime} \bar{F}_{i}^{-1}={ }_{H_{1}} P_{-1, i}$ both hold. We claim that $\left(\exists P_{-1, i}\right) D={ }_{H_{1}} P_{-1, i}$ if and only if (i) and (ii) hold. 
Suppose $D={ }_{H_{2}} P_{-1, i}$; then $D={ }_{H_{1}} \propto\left[P_{-1, i}\right]$. Now $\alpha\left[P_{-1, i}\right]$ must be $q$-reduced w.r.t. $\left(H_{1}, H_{2}\right)$ whence by GL $1, \propto\left[P_{-1, i}\right]$ is $P_{-1, i}^{*} \bar{F}_{i} q C_{-1}$. But then $L={ }_{H_{2}} C_{-1}$ and $D^{\prime}={ }_{H_{2}} P_{-1, i}^{*} \bar{F}_{i}$. The converse argument is trivial.

THEOR M 1 . Let $U$ and $V$ be any two words of $G_{0}$. Then $(\exists W) W^{-1} U W={ }_{G_{0}} V$ if and only if $(\exists W) W^{-1} \bar{r}\{U\} W={ }_{G_{0}} \bar{r}\{V\}$ where r-contraction is w.r.t. $\left(G_{0}, H_{1}\right)$.

Proof. This follows from GL 2 and Lemma 17.

LEMMA 18. (i) $\operatorname{Cond}_{J L B}\left(H, G_{3}\right.$, $\left.\{t, k\}\right)$ holds. (ii) $\operatorname{Cond}_{J L B}\left(G_{0}, H, q\right)$ holds.

Proof. (i) This is trivial. (ii) It is easy to see that $\left\{A_{i}\right\}$ and $\left\{B_{i}\right\}$ are sets of free generators.

LEM M $19 . \operatorname{Cond}_{J L B}\left(H, H_{2}, r_{i}\right)$ holds.

Proof. The argument of part (iii) (a) of Lemma 17 shows that $\left\{s_{b} x^{\varepsilon}, t, k\right\}$ is a set of free generators.

THEOREM 2. Let q-contraction be w.r.t. $\left(G_{0}, H\right)$ and r-contraction w.r.t. $\left(G_{0}, H_{1}\right)$ and let $U$ and $V$ be any two words of $G_{0}$. Then

(i) $(\exists W) W^{-1} U W={ }_{G_{0}} V$ if and only if $(\exists W) W^{-1} \bar{q}\{U\} W={ }_{G_{0}} \bar{q}\{V\}$;

(ii) if $U$ is r-contracted, then $\bar{q}\{U\}$ is r-contracted.

Proof. (i) This follows from GL 2 and Lemma 18. (ii) It suffices to consider the case in which $\bar{q}\{U\}$ is a primitive $q$-contraction of $U$; the general argument is completed by induction. Four possible subcases occur. These are

(1) $U$ is $U_{1} q^{-1} X q U_{2}$ with $X={ }_{H} A, \bar{q}\{U\}$ is $U_{1} \propto[B] U_{2}$;

(2) the dual of (1);

(3) $U$ is $X_{2} q U_{0} q^{-1} X_{1}$ with $X_{1} X_{2}={ }_{H} A, \bar{q}\{U\}$ is $\alpha[B] U_{0}$;

(4) the dual of (3).

We shall give the argument required for (1); the others are similar. So suppose $\bar{q}\{U\}$ is not $r$-contracted. We must consider the possible ways in which this could occur. It is clear that the appropriate $r_{i}^{-\varepsilon} D r_{i}^{\varepsilon}$ does not lie wholly within $U_{1}$ or within $U_{2}$ nor can it begin in $U_{2}$ and terminate in $U_{1}$. Moreover, $\alpha[B]$ is $r$-reduced w.r.t. $\left(G_{0}, H_{1}\right)$. The first possibility remaining is that $U_{1}$ is $U_{1}^{\prime} r_{i}^{-\varepsilon} D$ and $\alpha[B]$ is $\left(K_{i} r_{i}\right)^{\varepsilon} B^{\prime}$ where $D$ is $r$-free and $D K_{i}={ }_{H_{1}} P_{1, i}$ or $D={ }_{H_{1}} P_{-1, i}$ according as $\varepsilon= \pm 1$. We shall examine the case when $\varepsilon=1$ in detail.

Now $\alpha[A]$ must be $r_{i} F_{i} A^{\prime}$ where $A^{\prime} \sim B^{\prime}$. Since $X$ must be $r$-reduced w.r.t. $\left(G_{0}, H_{1}\right)$, it follows that $X$ is $r$-reduced w.r.t. $\left(H, H_{2}\right)$. The latter is also true of $\alpha[A]$ and since $X={ }_{H} \alpha[A]$, it follows that $X$ is $L r_{i} X^{\prime}$ where $L$ is $r$-free. Furthermore we obtain 
$\left(\exists C_{1}\right) L={ }_{H_{2}} C_{1}$. Thus $D q^{-1} L={ }_{H_{1}} P_{1, i} K_{i}^{-1} q^{-1} C_{1}$ which is impossible. A similar contradiction is obtained when $\varepsilon=-1$.

The second possibility is that $\alpha[B]$ is $B^{\prime}\left(K_{i} r_{i}\right)^{-\varepsilon}$ and $U_{2}$ is $D r_{i}^{\varepsilon} U_{2}^{\prime}$ where $D$ is $r$-free and $D={ }_{H_{1}} P_{1, i}$ or $K_{i}^{-1} D={ }_{H_{1}} P_{-1, i}$ accoording as $\varepsilon= \pm 1$. This case is dealt with in the same way as the first. Finally it is possible that $U_{1}$ is $U_{1}^{\prime} r_{i}^{-\varepsilon} D_{1}, \alpha[B]$ is $r$-free and $U_{2}$ is $D_{2} r_{i}^{e} U_{2}^{\prime}$ with $D_{1} \propto[B] D_{2}={ }_{H_{1}} P_{\varepsilon . i}$. But if $\alpha[B]$ is $r$-free, then $\alpha[B]$ is 1 . Hence $\alpha[A]$ is 1 whence $X={ }_{H_{2}} 1$. Then $D_{1} q^{-1} X q D_{2}={ }_{H_{1}} D_{1} D_{2}={ }_{H_{1}} P_{\varepsilon, i}$ which is impossible.

Soldtion 7. $(? X)(\exists A) X={ }_{H} A$ and $(? X)(\exists B) X={ }_{H} B$ are recursively solvable.

Proof. Defining $r$-reduction w.r.t. $\left(H, H_{2}\right)$ we can recursively compute $r[X]$. Let $A^{*}$ be $r$-parallel to $r[X]$. We claim that $(\exists A) X={ }_{H} A$ if and only if $r[X]={ }_{H} A^{*}$. To prove this it suffices to remark that if $X={ }_{H} A$, then $\alpha[A]$ is $A^{*}$. Since $\operatorname{Cond}_{J_{L B}}\left(G_{0}, H, q\right)$ holds, $H$ has a solvable word problem and the result follows.

TH Оов м 3. Let $t k$-, $q$ - and r-contraction be defined w.r.t. $\left(G_{0}, G_{2}\right),\left(G_{0}, H\right)$ and $\left(G_{0}, H_{1}\right)$ respectively. Also let $U$ and $V$ be any two words of $G_{0}$. Then (i) $(\exists W) W^{-1} U W={ }_{c_{0}} V$ if and only if $(\exists W) W-\bar{t} \overline{t k}\{U\} W={ }_{G_{0}} \overline{t k}\{V\}$; (ii) if $U$ is $q$-and $r$-contracted, then $\overline{t k}\{U\}$ is also $q$-and $r$-contracted.

Proof. (i) This follows from GL 2 and Lemmas 11 and 12. (ii) The argument required is similar to that given for part (ii) of Theorem 2.

Call a word $U$ normal if $U$ is $t k-, q$ - and $r$-contracted.

REDUCtion II. $(? U, V)(\exists W) W^{-1} U W={ }_{G_{0}} V \longrightarrow(? U, V$ normal $)(\exists W) W^{-1} U W=G_{0} V$.

Proof. This follows from GL 7, Theorems 1, 2 and 3, and Lemma 11, Solution 6 and Solution 7.

Call a word $U$ q.regular if $U$ is normal and has $q^{-1}$ as its final symbol.

REDUCTION III. (? $U, \quad V$ normal $)(\exists W) W^{-1} U W={ }_{G_{0}} V \longrightarrow(? U, V q$-regular $)(\exists A)$ $A^{-1} U A={ }_{G_{0}} V$ and $(? Y, Z$ normal $)(\exists W) W^{-1} Y W={ }_{G_{0}} Z$.

Proof. Let $O_{1}$ and $O_{2}$ be the two oracles which solve the latter problems. Given $U$ and $V$ normal, by GL 3 , if they are not $q$-circumparallel then they are not conjugate. So we need only consider pairs $U, V$ which are $q$-circumparallel. If $U$ and $V$ are $q$-free then we apply immediately to $\mathrm{O}_{2}$ which will provide the appropriate answer.

Now we need consider only those pairs $U, V$ which are not $q$-free. Let $\left\{\left(U_{i}, V_{i}\right)\right\}$ be a listing of all possible pairs such that $U_{i}$ and $V_{i}$ are $q$-regular cyclic permutations of $U$ and $V$ respectively. Also let $\left\{\left(U_{k}, V_{k}\right)\right\}$ be a listing of all possible pairs $U_{k}$ and $V_{k}$ such that 
$U_{k}$ and $V_{k}$ are cyclic permutations of $U$ and $V$ respectively which terminate in $q$. Each listing is certainly finite. We can use $O_{1}$ on each pair $\left(U_{i}, V_{i}\right)$ to determine whether or not ( $\exists A) A^{-1} U_{i} A={ }_{G_{0}} V_{i}$. But it follows from GL 6 that we can also use $O_{1}$ to determine for each pair $\left(U_{k}, V_{k}\right)$ whether or not $(\exists B) B^{-1} U_{k} B={ }_{G_{0}} V_{k}$ (for if $U_{k}^{\prime} q$ is normal then $U_{k}^{\prime-1} q^{-1}$ is also normal). By GL 3, a negative answer to all of these problems means that $U$ and $V$ are not conjugate. But also a positive answer to any single one means that $U$ and $V$ are conjugate.

$\S 3$. Define $\operatorname{Conj}\left(U, V, W, E^{*}, E, p\right)$ to mean that $V$ is a right conjugate of $U$ w.r.t. $\left(E^{*}, E, p\right)$ by $W$. Call a word $U$ standard if it is $q$-regular and not $r$-free.

REDUCTION IV. (?U, V q-regular $)(\exists A) A^{-1} U A={ }_{G_{0}} V \longrightarrow(? U, V$ standard $)(\exists A)$ Conj $\left(U, V, A, G_{0}, H_{1}, r\right)$ and (?D, E q-regular $)(\exists A) A^{-1} D A={ }_{G_{0}} E$

Proof. It follows from GL 3 that if $U$ and $V$ are conjugate, then either both are $r$-free or both contain $r$-symbols. If both are $r$-free we apply to the oracle for the second problem listed above. If $U$ and $V$ both contain $r$-symbols, the sufficiency of the oracle for the first problem follows from GL 4, GL 5 and the fact that if $A$ is $\alpha$-reduced, then $A$ is $r$-reduced w.r.t. $\left(G_{0}, H_{1}\right)$.

In this section we concentrate on the problem (?D, E q-regular) $(\exists A) A^{-1} D A={ }_{G_{0}} E$. Let $D$ have the form $L_{1} q^{\varepsilon_{1}} L_{2} q^{\varepsilon_{3}} \ldots L_{m} q^{e_{m}}$; we call $L_{1}, L_{2}, \ldots, L_{m}$ the $q$-factors of $D$. We say $D$ is factor reduced if each $L_{u}$ is stk-reduced w.r.t. $\left(H_{2}, F(x)\right)$. Also we say

(1) $L_{u}$ is of type $\alpha$ if $\varepsilon_{u-1}=-1, \varepsilon_{u}=1$;

(2) $L_{u}$ is of type $\beta$ if $\varepsilon_{u-1}=1, \varepsilon_{u}=-1$;

(3) $L_{u}$ is of type $\gamma$ if $\varepsilon_{u-1}=-1, \varepsilon_{u}=-1$;

(4) $L_{u}$ is of type $\delta$ if $\varepsilon_{u-1}=1, \varepsilon_{u}=1$

(where we consider $\varepsilon_{m}$ to be $\varepsilon_{1-1}$ ).

Lемма 20. (?D, $E$ q-regular $)(3 A) A^{-1} D A={ }_{G_{0}} E \rightarrow(? D, E$ q-regular, factor reduced $)$ ( $\exists A) A^{-1} D A={ }_{G_{0}} E$.

Proof. Computation of $s t k[L]$ is a recursive process.

For the remainder of this section we shall always assume that $D$ and $E$ are factor reduced.

Lемм 21 . Let $D$ and $E$ be q-reduced w.r.t. $\left(G_{0}, H\right)$ and suppose that $D$ is $L_{1} q^{\varepsilon_{1}} L_{2} q^{\varepsilon_{2}} \ldots L_{m} q^{\varepsilon_{m}}$ and that $E$ is $M_{1} q^{\eta_{1}} M_{2} q^{\eta_{z}} \ldots M_{n} q^{\eta_{n}}$. If $D={ }_{H_{1}} E$, then $m=n, \varepsilon_{u}=\eta_{u}$ and $L_{u}={ }_{H_{2}} M_{u}, u=1,2, \ldots, m$. 
Proof. $D$ and $E$ must in fact be $q$-reduced w.r.t. $\left(H_{1}, H_{2}\right)$. The result follows by an obvious inductive argument using $q$ as stable letter w.r.t. $\left(H_{1}, H_{2}\right)$.

LemMA 22. Let $D$ and $E$ be q-regular and q-parallel with $q$-factors $L_{1}, L_{2}, \ldots, L_{n}$ and $M_{1}, M_{2}, \ldots, M_{n}$ respectively. If $A^{-1} D A={ }_{G_{0}} E$ and $A \sim B$, then
( $\alpha) A^{-1} L_{u} A={ }_{H} M_{u}$ or
( $\beta) B^{-1} L_{u} B={ }_{H} M_{u}$
or

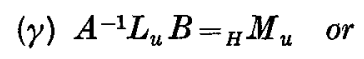
(ס) $B^{-1} L_{u} A={ }_{H} M_{u}$

according as $L_{u}$ and $M_{u}$ are both of type $\alpha, \beta, \gamma$ or $\delta$.

Proof. We proceed by induction w.r.t. $l_{r}(A)$. If $l_{r}(A)=0$, then $D={ }_{G_{0}} E$ whence $D==_{H_{1}} E$. The result is immediate by Lemma 21. So suppose $l_{r}(A)>0$; if $A$ is not $\alpha$-reduced then we can replace $A$ by $\alpha[A]$ and the result is immediate from the inductive hypothesis. Assume then that $A$ is $\alpha$-reduced and write $A$ as $A_{i}^{\varepsilon} A^{\prime}$. We give a detailed argument only for the case when $\varepsilon=1$.

Now $A^{-1} D A$ is $A^{\prime-1} \bar{F}_{i}^{-1} r_{i}^{-1} D r_{i} \bar{F}_{i} A^{\prime}={ }_{G_{0}} E$ and hence $\left(\exists P_{1, i}\right) D={ }_{H_{1}} P_{1, i}$ since $E$ is $r$-free. Since $\alpha\left[P_{1, i}\right]$ must be $q$-reduced, $\alpha\left[P_{1, i}\right]$ is $q$-parallel to $D$. Thus $\alpha\left[P_{1, i}\right]$ is $C_{1}^{(1)}\left(q K_{i}\right)^{\varepsilon_{1}} \ldots\left(q K_{i}\right)^{\varepsilon_{n-1}} C_{1}^{(n)} K_{i}^{-1} q^{-1} C_{1}^{(n+1)}$. But it follows immediately that $C_{1}^{(n+1)}$ is 1 and hence by Lemma 21 we have

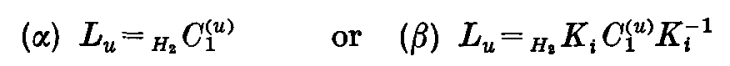

or $\quad(\gamma) L_{u}={ }_{H_{3}} C_{1}^{(u)} K_{i}^{-1}$ or $\quad(\delta) L_{u}={ }_{H_{3}} K_{i} C_{1}^{(u)}$

as is appropriate. The primitive $r$-reduction $\bar{r}\left[A^{-1} D A\right]$ is

$$
A^{\prime-1} \bar{F}_{i}^{-1} C_{-1}^{(1)}\left(\bar{F}_{i} q\right)^{\varepsilon_{1}} \ldots\left(\bar{F}_{i} q\right)^{\varepsilon_{n-1}} C_{-1}^{(n)} q^{-1} \bar{F}_{i}^{-1} \bar{F}_{i} A^{\prime}
$$

Then $\bar{r}\left[A^{-1} D A\right]={ }_{G_{0}} A^{\prime-1} D_{1} A^{\prime}$ where we write $D_{1}$ for

$$
\bar{F}_{i}^{-1} C_{-1}^{(1)}\left(\bar{F}_{i} q\right)^{\varepsilon_{1}} \ldots\left(\bar{F}_{i} q\right)^{\varepsilon_{n-1}} C_{-1}^{(n)} q^{-1} .
$$

We want to show that $D_{1}$ is $q$-regular. Certainly $D_{1}$ is $r$-contracted since it is $r$.free. If $D_{1}$ is not $q$-contracted, then either $(\alpha)$ for some $u,(\exists A) \bar{F}_{i}^{-1} C_{-1}^{(u)} \bar{F}_{i}={ }_{H} A$ or $(\beta)$ for some $u,(\exists B) C_{-1}^{(u)}={ }_{H} B$. In case $(\alpha)$, since $\bar{F}_{i}^{-1} C_{-1}^{(u)} \bar{F}_{i}$ is $r$-free, $A={ }_{H} 1$. Thus $C_{-1}^{(u)}={ }_{H} 1$ whence $C_{-1}^{(u)}$ is $1\left(C_{1}^{(u)}\right.$ is part of $\left.\alpha\left[P_{1, i}\right]\right)$. This gives $L_{u}={ }_{H_{2}} 1$ which is impossible

Case $(\beta)$ will yield the same contradication.

Suppose that $D_{1}$ is not $t k$-contracted w.r.t. $\left(G_{0}, G_{2}\right)$. It is easy to see that the appropriate subword $t^{-\varepsilon} C t^{\varepsilon}$ or $k^{-\varepsilon} C k^{\varepsilon}$ must lie within a $q$-factor. But the occurrence of such a subword within a $q$-factor implies, after a short argument, that the $q$-factor is not stk- 
reduced w.r.t. $\left(H_{2}, F^{\prime}(x)\right)$. But $C_{1}^{(u)}$ and hence $C_{-1}^{(u)}$ (for all $u$ ) are $\alpha$-reduced whence it follows that $C_{-1}^{(u)}, \bar{F}_{i}^{-1} C_{-1}^{(u)}$ and $C_{-1}^{(u)} \bar{F}_{1}$ are all $s t k$-reduced. This is contradictory.

We can thus apply the inductive hypothesis to $D_{1}$ and $E$ giving

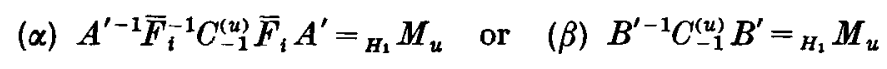

or

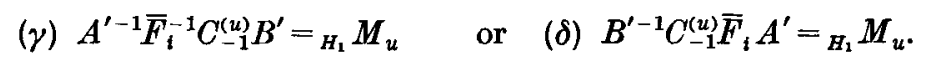

The lemma now follows easily.

For any word $L$, let $m(L)$ be the number of occurrences of positive $s$-symbols in $L$ and $n(L)$ be the number of occurrences of negative $s$-symbols in $L$. Then the s-signature of $L$ is $\sigma(L)=m(L)-n(L)$. For example, if $L$ is $s_{1} s_{2}^{-1} s_{3}$, then $m(L)=2$ and $n(L)=1$ whence $\sigma(L)=1$.

Leмма 23. Let $C_{\varepsilon}$ be $\alpha$-reduced with s-signature $\sigma$. Then (i) if $x^{-n} C_{\varepsilon} x^{m}={ }_{H_{8}} C_{\varepsilon}$ then $m=2^{\sigma} n$; (ii) if $x^{-n} C_{-\varepsilon} x^{m}={ }_{H_{3}} C_{\varepsilon}$, then $m=2^{\sigma} n+\varepsilon\left(2^{\sigma+1}-2\right)$.

Proof. By induction on $l_{\text {stk }}\left(C_{\varepsilon}\right)$.

Lемма 24. (i) Let $C_{\varepsilon}$ be stk-parallel to the positive word $\Psi$ on the s-symbols. Then $C_{\varepsilon}={ }_{H_{2}} \Psi x^{\varepsilon\left(2^{\varepsilon}-1\right)}$ where $c=l\left(\Psi^{\dagger}\right)$. (ii) Let $C_{\varepsilon}$ be stk-parallel to $\Psi^{-1}$ where $\Psi$ is a positive word on the s-symbols. Then $C_{\varepsilon}={ }_{H_{\mathrm{a}}} x^{\left.-\varepsilon^{(2 c}-1\right)} \Psi^{-1}$, where $c=l(\Psi)$.

Proof. By induction on $c$.

LeMma 25. Let $L$ and $M$ be stk-reduced. Then (i) if $A$ is $\alpha$-reduced and $A^{-1} L B={ }_{H} M$ where $A \sim B$, then $l_{r}(A) \leqslant 2$; (ii) if $A$ is $\alpha$-reduced and $B^{-1} L A={ }_{H} M$ where $A \sim B$, then $l_{r}(A) \leqslant 2$.

Proof. (i) We shall show that if $A$ is $\alpha$-reduced and $l_{\tau}(A)=3$, then $r\left[A^{-1} L B\right]$ cannot be $r$-free $\left(r\right.$-reduction w.r.t. $\left.\left(H, H_{2}\right)\right)$. The result follows from this. For if $A$ is $\alpha$-reduced and $A^{-1} L B={ }_{H} M$, then $r\left[A^{-1} L B\right]$ must be $r$-free.

We consider all 8 possible values for $A$ with $l_{r}(A)=3$ and $A \alpha$-reduced. These are
(1) $r_{i} \bar{F}_{i} r_{j} \bar{F}_{j} \bar{F}_{k}^{-1} r_{k}^{-1}, \quad j \neq k$;
(2) $r_{i} \bar{F}_{i} \bar{F}_{j}^{-1} r_{j}^{-1} r_{k} \bar{F}_{k}, i \neq j, \quad j \neq k$;
(3) $r_{i} \bar{F}_{i} \bar{F}_{j}^{-1} r_{j}^{-1} \bar{F}_{k}^{-1} r_{k}^{-1}, \quad i \neq j$;
(4) $\bar{F}_{i}^{-1} r_{i}^{-1} r_{j} \bar{F}_{j} \bar{F}_{k}^{-1} r_{k}^{-1}, i \neq j, \quad i \neq k$;
(5) $\bar{F}_{i}^{-1} r_{i}^{-1} \bar{F}_{j}^{-1} r_{j}^{-1} r_{k} \bar{F}_{k}, \quad j \neq k$;
(6) $\bar{F}_{i}^{-1} r_{i}^{-1} r, \bar{F}, r_{k} \bar{F}_{k}, \quad i \neq j$;
(7) $r_{i} \bar{F}_{i} r_{j} \bar{F}_{j} r_{k} \bar{F}_{k}$;
(8) $\bar{F}_{i}^{-1} r_{i}^{-1} \bar{F}_{j}^{-1} r_{j}^{-1} \bar{F}_{k}^{-1} r_{k}^{-1}$.

Cases (1), (2), (3) and (4). It suffices to show that if $A_{0}$ is $r_{i} \bar{F}_{i} \bar{F}_{j}^{-1} r_{j}^{-1}, i \neq j$, then $r\left[A_{0}^{-1} L B_{0}\right]$, where $A_{0} \sim B_{0}$, is not $r$-free. Suppose it is; we shall follow through the computation of $r\left[A_{0}^{-1} L B_{0}\right]$ and obtain a contradiction. 
Stage $I$. For the first primitive $r$-reduction to occur, it is necessary that $\left(\exists C_{1}^{(1)}\right) L K_{i}$ $={ }_{H_{2}} C_{1}^{(1)}$. Upon performing this first stage, we obtain the word $r_{j} \bar{F}_{j} \bar{F}_{i}^{-1} \alpha\left[C_{-1}^{(1)}\right] r_{j}^{-1} K_{j}^{-1}$.

Stage II. A second pair of $r$-symbols must be eliminated. Hence $\left(\exists C_{-1}^{(2)}\right) \bar{F}_{j} \bar{F}_{i}^{-1} \alpha\left[C_{-1}^{(1)}\right]$ $={ }_{H_{2}} C_{-1}^{(2)}$ whence it follows that $\bar{F}_{i}^{-1} \alpha\left[C_{-1}^{(1)}\right]={ }_{H_{z}} \bar{F}_{j}^{-1} \alpha\left[C_{-1}^{(2)}\right]$. Now both words in this last equation are $s t k$-reduced and hence are $s t k$-parallel. Since $F_{i}$ and $F_{j}$ are distinct, we must obtain either
(a) $\Psi \propto\left[C_{-1}^{(1)}\right]={ }_{H_{9}} \alpha\left[C_{-1}^{(2)}\right]$ or
(b) $\alpha\left[C_{-1}^{(1)}\right]={ }_{H z} \Psi \alpha\left[C_{-1}^{(2)}\right]$

where $\Psi^{*}$ is a non-empty, positive word on the $s$-symbols.

We examine (a) in detail. It is clear that $\alpha\left[C_{-1}^{(2)}\right]$ must have the form $C_{-1}^{*} \alpha\left[C_{-1}^{(1)}\right]$ where $C_{-1}^{*}$ is $s t k$-parallel to $\Psi$. Hence $\Psi={ }_{H_{2}} C_{-1}^{*}$; but by Lemma $24 C_{-1}^{*}={ }_{H_{2}} \Psi x^{-\left(2^{c}-1\right)}$ where $c=l(\Psi)$. This implies that $x^{-\left(2^{c}-1\right)}={ }_{H_{2}} 1$ whence $x^{-\left(2^{c}-1\right)}=1$ in $F(x)$. Since $c \neq 0$, this is a contradiction. The argument for (b) is almost identical. We have thus disposed of (1), (2), (3) and (4).

Cases (5) and (6). These are dealt with by applying the argument dual to that given above to show that if $B_{0}$ is $r_{i}^{-1} K_{i}^{-1} K_{j} r_{j}$, then $r\left[A_{0}^{-1} L B_{0}\right]$, where $A_{0} \sim B_{0}$, cannot be $r$-free.

Case (7). We follow through the computation of $r\left[A^{-1} L B\right]$ in the same manner as above.

Stage $I .\left(\exists C_{1}^{(1)}\right) L K_{i}={ }_{H_{2}} C_{1}^{(1)}$ yielding as primitive $r$-reduction

$$
\bar{F}_{k}^{-1} r_{k}^{-1} \bar{F}_{j}^{-1} r_{j}^{-1} \bar{F}_{i}^{-1} \alpha\left[C_{-1}^{(1)}\right] K_{j} r_{j} K_{k} r_{k}
$$

Stage II. $\left(\exists C_{1}^{(2)}\right) \bar{F}_{i}^{-1} \alpha\left[C_{-1}^{(1)}\right] K_{j}={ }_{H_{\mathrm{s}}} C_{1}^{(2)}$ yielding as $r$-reduction $\bar{F}_{k}^{-1} r_{k}^{-1} \bar{F}_{j}^{-1} \alpha\left[C_{-1}^{(2)}\right] K_{k} r_{k}$.

Stage III. $\left(\exists C_{1}^{(3)}\right) \bar{F}_{j}^{-1} \alpha\left[C_{-1}^{(2)}\right] K_{k}={ }_{H_{2}} C_{1}^{(3)}$ must hold. From this we obtain

$$
\bar{F}_{j}^{-1} \alpha\left[C_{-1}^{(2)}\right]={ }_{H_{2}} \alpha\left[C_{1}^{(3)}\right] K_{k}^{-1} .
$$

These last are both $s t k$-reduced and hence are $s t k$-parallel. Thus $\bar{F}_{j}^{-1} \alpha\left[C_{-1}^{(2)}\right]$ must have the form $\bar{F}_{j}^{-1} C_{-1}^{\prime} C_{-1}^{*}$ where $C_{-1}^{*}$ is stk-parallel to $K_{k}^{-1}$. Hence by Lemma 24

$$
\bar{F}_{j}^{-1} \alpha\left[C_{-1}^{(2)}\right]={ }_{H_{2}} \bar{F}_{j}^{-1} C_{-1}^{\prime} x^{2^{d-1}} K_{k}^{-1}, \text { where } d=l\left(K_{k}\right)
$$

But also from Stage III we see that $\alpha\left[C_{1}^{(3)}\right] K_{k}^{-1}$ must be $C_{1} C_{1}^{\prime} K_{k}^{-1}$ where $C_{1}^{\prime} \sim C_{-1}^{\prime}$ and $C_{1}^{\tilde{1}}$ is stk parallel to $\bar{F}_{j}^{-1}$. This gives $\alpha\left[C_{1}^{(3)}\right] K_{k}^{-1}={ }_{H_{2}} \bar{F}_{j}^{-1} x^{2^{c}-1} C_{1}^{\prime} K_{k}^{-1}$ where $c=l\left(F_{j}\right)$. Then $x^{-(2 c-1)} C_{-1}^{\prime} x^{2 d-1}={ }_{H_{3}} C_{1}^{\prime}$ whence $2^{d}-1=2^{\sigma}\left(2^{c}-1\right)+\left(2^{\sigma+1}-2\right)$ where $\sigma=\sigma\left(C_{1}^{\prime}\right)$. Since $c \neq 0$ and $d \neq 0, \sigma\left(C_{1}^{\prime}\right)=0$ and thus $\sigma\left(\alpha\left[C_{-1}^{(2)}\right]\right)=-d$ and $\sigma\left(\alpha\left[C_{1}^{(3)}\right]\right)=c$. A similar analysis from Stage II gives $\sigma\left(\alpha\left[C_{-1}^{(1)}\right]\right)=-a$ and $\sigma\left(\alpha\left[C_{1}^{(2)}\right]\right)=b$ where $a=l\left(K_{j}\right)$ and $b=l\left(F_{i}\right)$ and $a \neq 0, b \neq 0$. But this implies that $b=-d$ which is impossible.

Case (8). The argument required is the dual of that for case (7).

(ii) The proof is dual to that for (i). 
THEOREM 4. Let $D$ and $E$ be q-regular and suppose $A^{-1} D A={ }_{G_{0}} E$ with $A \alpha$-reduced. If $D$ contains a $q$-factor of type $\gamma$ or type $\delta$, then $l_{r}(A) \leqslant 2$.

Proof. This is immediate from Lemmas 22 and 25 since $D$ and $E$ must be $q$-parallel.

Let $D$ be $q$-regular; call $D$ q-alternating if $D$ has no $q$-factors of type $\gamma$ or type $\delta$.

L Е Mм А 26. Let $L$ and $M$ be stk-reduced and suppose $\sigma(L) \neq 0$. Then (i) if $A$ is $\alpha$-reduced and $A^{-1} L A={ }_{H} M$, then $l_{r}(A) \leqslant 2$; (ii) if $B$ is $\alpha$-reduced and $B^{-1} L B=M$, then $l_{r}(B) \leqslant 2$.

Proof. (i) We show, much as in Lemma 25, that if $A$ is $\alpha$-reduced and $l_{r}(A)=3$, then $r\left[A^{-1} L A\right]$ cannot be $r$-free.

Cases (1), (2), (3) and (4) (see Lemma 25). We show that if $A_{0}$ is $r_{i} \bar{F}_{i} \bar{F}_{j}^{-1} r_{j}^{-1}$, then $r\left[A_{0}^{-1} L A_{0}\right)$ cannot be $r$-free. We follow the computation of $r\left[A_{0}^{-1} L A_{0}\right]$.

Stage $I .\left(\exists C_{1}^{(1)}\right) L={ }_{H_{2}} C_{1}^{(1)}$ yielding as $r$-reduction the word $r_{j} \bar{F}_{j} \bar{F}_{i}^{-1} \propto\left[C_{-1}^{(1)}\right] \bar{F}_{i} \bar{F}_{j}^{-1} r_{j}^{-1}$.

Stage II. $\left(\exists C_{-1}^{(2)}\right) \bar{F}_{j} \bar{F}_{i}^{-1} \alpha\left[C_{-1}^{(1)}\right] \bar{F}_{i} \bar{F}_{j}^{-1}={ }_{H_{2}} C_{-1}^{(2)}$ which implies that $\bar{F}_{j}^{-1} \alpha\left[C_{-1}^{(1)}\right] \bar{F}_{i}={ }_{H_{2}}$ $\bar{F}_{j}^{-1} \alpha\left[C_{-1}^{(2)}\right] \bar{F}_{j}$. Both these last are $s t k$-reduced words and so they must be $s t k$-parallel. Hence either (a) $\Psi \alpha\left[C_{-1}^{(1)}\right] \Psi^{-1}={ }_{H_{2}} \alpha\left[C_{-1}^{(2)}\right]$ or (b) $\alpha\left[C_{-1}^{(1)}\right]={ }_{H_{2}} \Psi \alpha\left[C_{-1}^{(2)}\right] \Psi^{-1}$ where $\Psi$ is nonempty $\left(i \neq j\right.$ implies that $F_{i}$ is distinct from $\left.F_{j}\right)$. We examine (a) in detail.

Now $\alpha\left[C_{-1}^{(2)}\right]$ must have the form $C_{-1}^{*} \alpha\left[C_{-1}^{(1)}\right] C_{-1}^{*-1}$ with $C_{-1}^{*}={ }_{H_{2}} \Psi x^{-\left(2^{c-1}\right)}$ where $c=l(\Psi)$. Then $\alpha\left[C_{-1}^{(1)}\right]={ }_{H_{2}} x^{-(2 c-1)} \alpha\left[C_{-1}^{(1)}\right] x^{2 c-1}$ whence $2^{c}-1=2^{\sigma}\left(2^{c}-1\right)$ where $\sigma=\sigma\left(\alpha\left[C_{-1}^{(1)}\right]\right)$. Hence $\sigma\left(\alpha\left[C_{-1}^{(1)}\right]\right)=0$. Since $L$ is stk-parallel to $\alpha\left[C_{1}^{(1)}\right]$, we have $\sigma(L)=0$ which is impossible. Case (b) is similar.

Cases (6) and (7). We show that if $A_{0}$ is $r_{i} \bar{F}_{i} r_{j} \bar{F}_{f}$, then $r\left[A_{0}^{-1} L A_{0}\right]$ cannot be $r$-free. We proceed as before.

Stage $I,\left(\exists C_{1}^{(1)}\right) L={ }_{H_{2}} C_{1}^{(1)}$.

Stage II. $\left(\exists C_{1}^{(2)}\right) \bar{F}_{i}^{-1} \alpha\left[C_{-1}^{(1)}\right] \bar{F}_{i}={ }_{H_{3}} C_{1}^{(2)}$. Hence $\alpha\left[C_{1}^{(2)}\right]$ is $C_{1}^{*} \alpha\left[C_{1}^{(1)}\right] C_{1}^{*-1}$ with $C_{1}^{*}={ }_{H_{3}}$ $\bar{F}_{i}^{-1} x^{2^{c-1}}$, where $c=l\left(F_{i}\right) \neq 0$. This gives $x^{2 c-1} \alpha\left[C_{1}^{(1)}\right] x^{-(2 c-1)}={ }_{H_{2}} \alpha\left[C_{-1}^{(1)}\right]$. Using part (ii) of Lemma 23, we again obtain $\sigma(L)=0$ which is contradictory.

Cases (5) and (8). The argument required is a slight variation of those already used.

THEOREM 5. Let $D$ and $E$ be q-regular, q-alternating and suppose $A^{-1} D A={ }_{G_{0}} E$ where $A$ is $\alpha$-reduced. If $D$ or $E$ has a $q$-factor whose s-signature is non-zero, then $l_{r}(A) \leqslant 2$.

Proof. $D$ and $E$ must be $q$-parallel; the result follows from Lemmas 22 and 26.

Let $L$ be any $r$-free, $q$-free word and let $\pi_{s}[L]$ be the $s$-projection of $L$. Then there is a sequence $S_{1}, S_{2}, \ldots, S_{n}$ of words on the $s$-symbols such that (i) $S_{0}$ is 1 , (ii) $S_{i+1}$ is $S_{i} s_{b_{i}+1}+1$ and (iii) $S_{n}$ is $\pi_{s}[L]$. 
Let $\sigma_{i}=\sigma\left(S_{i}\right)$; call $\left(\sigma_{0}, \sigma_{1}, \ldots, \sigma_{n}\right)$ the $s$-distribution of $L$. Let $v(L)=-\min _{0 \leqslant i \leqslant n}\left\{\sigma_{i}\right\}$ and call $\nu(L)$ the $s$-index of $L$.

LEM M 27. Let $L$ be stk-reduced with s-signature $\sigma$ and s-index $v$. Then $x^{-2 p} L x^{2 y+\sigma}={ }_{H_{\mathrm{a}}} L$.

Proof. By induction on $l_{\text {stk }}(L)$.

Lемма 28. Let $C_{\varepsilon}$ be stk-reduced with s-signature $\sigma=0$ and s-index $v$. Then $x^{-2^{v}} C_{\varepsilon} x^{2 v}={ }_{H_{\varepsilon}} C_{\varepsilon}$

Proof. From Lemma 27.

L EMM 29. Let $L$ be stk-reduced and have s-index $v$. If $x^{-n} L x^{m}={ }_{H z} L$, then $2^{y} \mid n$.

Proof. By induction on $l_{s t k}(L)$.

L Е M M 30. Let $C_{\varepsilon}$ be stk-reduced with s-index $\nu$ and s-signature $\sigma=0$. If $x^{-m} C_{\varepsilon} x^{m}={ }_{H_{z}} C_{\varepsilon}$, then $2^{v} \mid m$.

Proof. From Lemma 29.

LEMMA 31. For any $C_{1}, x^{2} C_{-1} x^{-2}={ }_{H_{2}} C_{1}$.

Proof. We remark that (i) $x^{2} t^{\eta} x^{-2}={ }_{H_{2}} t^{\eta}$ and $x^{2} k^{\eta} x^{-2}={ }_{H_{3}} k^{\eta}$; (ii) $x^{2} s_{b} x^{-1} x^{-2}={ }_{H_{2}}$ $s_{b} x^{4} x^{-3}={ }_{H_{z}} s_{b} x$; (iii) $x^{2} x s_{b}^{-1} x^{-2}={ }_{H_{3}} x^{3} x^{-4} s_{b}^{-1}={ }_{H_{2}} x^{-1} s_{b}^{-1}$.

The proof is by an obvious induction.

LEMM 32. Let $L$ and $M$ be stk-reduced and suppose $\sigma(L)=0$ and $v(L)>0$. Then (i) if $A$ is $\alpha$-reduced and $A^{-1} L A={ }_{H} M$, then $l_{r}(A) \leqslant 2$; (ii) if $B$ is $\alpha$-reduced and $B^{-1} L B={ }_{H} M$, then $l_{r}(B) \leqslant 2$.

Proof. (i) We proceed much as in Lemma 26.

Cases (1), (2), (3), and (4). The argument is identical down to the equation $\alpha\left[C_{-1}^{(1)}\right]={ }_{H_{2}} x^{-\left(2^{c}-1\right)} \alpha\left[C_{-1}^{(1)}\right] x^{2^{c}-1}$ of case (a). From this we conclude that $2^{v} \mid 2^{c}-1$ where $\boldsymbol{v}=\nu\left(\alpha\left[C_{-1}^{(1)}\right]\right)$. Hence $\nu\left(\alpha\left[C_{-1}^{(1)}\right]\right)=0$. But $L={ }_{H_{2}} \alpha\left[C_{+1}^{(1)}\right]$ whence $\nu(L)=0$.

In case $(\mathrm{b})$ we obtain $v\left(\alpha\left[C_{-1}^{(2)}\right]\right)=0$. Now $\alpha\left[C_{-1}^{(1)}\right]$ is stk-parallel to $\Psi^{p} \alpha\left[C_{-1}^{(2)}\right] \Psi^{-1}$ and so $v\left(\alpha\left[C_{-1}^{(1)}\right]=0\right.$ whence $v(L)=0$. In both cases (a) and (b) we thus obtain contradictions.

Cases (6) and (7). Arguing as in Lemma 26, we obtain the equation

$$
x^{2^{c-1}} \alpha\left[C_{1}^{(1)}\right] x^{-\left(2^{c-1}\right)}={ }_{H_{2}} \alpha\left[C_{-1}^{(1)}\right] .
$$

Hence, by Lemma 31, $x^{2^{c}+1} \alpha\left[C_{1}^{(1)}\right] x^{-\left(2^{c}+1\right)}={ }_{H_{2}} \alpha\left[C_{1}^{(1)}\right]$. From this we get $2^{v} \mid 2^{c}+1$ where 
$v=v\left(\alpha\left[C_{1}^{(1)}\right]\right)$. But this means that $v=0$ whence it follows that $v(L)=0$ which is contradictory.

Cases (5) and (8). The argument required is similar to that for cases (6) and (7).

(ii) This is proved dually to (i).

Theorem 6. Let $D$ and $E$ be q-regular and q-alternating and suppose $A^{-1} D A={ }_{G_{0}} E$ with $A \alpha$-reduced. If either $D$ or $E$ has a q-factor with s-signature $\sigma=0$ and s-index $\nu>0$, then $l_{r}(A) \leqslant 2$.

Proof. This is immediate from Lemmas 22 and 32.

LEMMA 33. If $L$ is stk-reduced with $\sigma(L)=0$ and $\nu(L)=0$, then $\left(\exists C_{1}\right) L={ }_{H_{2}} C_{1}$ if and only if $\left(\exists C_{-1}\right) L={ }_{H_{\mathbf{3}}} C_{-1}$.

Proof. $L={ }_{H_{1}} C_{1}$ if and only if $L={ }_{H_{2}} \alpha\left[C_{1}\right]$. Now $\alpha\left[C_{1}\right]$ must be $s t k$-parallel to $L$ whence $\sigma\left(\alpha\left[C_{1}\right]\right)=0$ and $\nu\left(\alpha\left[C_{1}\right]\right)=0$. Hence $x^{2} \alpha\left[C_{-1}\right] x^{-2}={ }_{H_{3}} \alpha\left[C_{1}\right]$ and $x^{2} \alpha\left[C_{-1}\right] x^{-2}={ }_{H_{3}} \alpha\left[C_{-1}\right]$. The lemma now follows.

In the case when $\sigma(L)=0$ and $\nu(L)=0$ the two conditions $\left(\exists C_{1}\right) L={ }_{H_{3}} C_{1}$ and $\left(\exists C_{-1}\right)$ $L={ }_{H_{2}} C_{-1}$ are thus equivalent. We therefore write $\left(\exists C_{\varepsilon}\right) L={ }_{H_{3}} C_{\varepsilon}$ for these and interpret $\varepsilon$ as 1 or -1 as we wish.

Lемма 34. Let $L$ and $M$ be stk-reduced and suppose (1) $\sigma(L)=0 ;(2) v(L)=0$; (3) $\left.\sim\left[\exists C_{\varepsilon}\right) L={ }_{H_{z}} C_{\varepsilon}\right]$.

(i) If $A$ is $\alpha$-reduced and $A^{-1} L A={ }_{H} M$, then $l_{r}(A) \leqslant 3$.

(ii) If $B$ is $\alpha$-reduced and $B^{-1} L B={ }_{H} M$, then $l_{r}(B) \leqslant 3$.

Proof. (i) Once again we examine the computation of $r\left[A^{-1} L A\right]$. We claim that $A$ must be $\bar{F}_{i}^{-1} r_{i}^{-1} A^{\prime}$; for suppose not. Then $\left({ } C_{1}\right) L={ }_{H_{2}} C_{1}$ which contradicts (3). Thus $A$ is $\bar{F}_{i}^{-1} r_{i}^{-1} A^{\prime}$ whence $\left({ }^{3} C_{-1}\right) \bar{F}_{i} L \bar{F}_{i}^{-1}={ }_{H_{2}} C_{-1}$ and $A^{\prime-1} \alpha\left[C_{1}\right] A^{\prime}={ }_{H} M$. If we can prove that $\sigma\left(\alpha\left[C_{1}\right]\right)=0$ and $v\left(\alpha\left[C_{1}\right]\right)>0$, then the lemma follows from Lemma 32. Since $L={ }_{H_{z}}$ $\bar{F}_{i}^{-1} \alpha\left[C_{-1}\right] \bar{F}_{i}$, it is clear that $\sigma\left(\alpha\left[C_{1}\right]\right)=0$. Suppose that $v\left(\alpha\left[C_{1}\right]\right)=0$; then $x^{-1} \alpha\left[C_{-1}\right] x=_{H_{3}}$ $\alpha\left[C_{-1}\right]$ whence $x^{-\left(2^{c}-1\right)} \alpha\left[C_{-1}\right] x^{2^{c-1}}=_{H_{2}} \alpha\left[C_{-1}\right]$ where $c=l\left(F_{i}\right)$. If $C_{-1}^{*}$ is $s t k$-parallel to $\bar{F}_{i}^{-1}$, then it follows that $C_{-1}^{*} \alpha\left[C_{-1}\right] C_{-1}^{*-1}={ }_{H_{2}} L$ which contradicts (3).

(ii) This is dual to (i).

Tнеовем 7. Let $D$ and $E$ be q-regular and q-alternating and suppose that $A^{-1} D A=G_{0} E$ where $A$ is $\alpha$-reduced. If either $D$ or $E$ has a $q$-factor $L$ such that $\sigma(L)=0, v(L)=0$ and $\sim\left[\left(\exists C_{\varepsilon}\right) L={ }_{H_{2}} C_{\varepsilon}\right]$, then $l_{r}(A) \leqslant 3$.

Proof. This is immediate from Lemmas 22 and 34. 
Call a word $D$ symmetric if $D$ is $q$-regular and $q$-alternating and every $q$-factor $L_{u}$ of $D$ satisfies the conditions (1) $L_{u}$ is stk-reduced, (2) $\sigma\left(L_{u}\right)=0$, (3) $v\left(L_{u}\right)=0$ and (4) ( $\left.\exists C_{\varepsilon}\right) L_{u}={ }_{H_{2}} C_{\varepsilon}$. If $D$ is symmetric with $n q$-factors, $\left({ }^{1}\right)$ a representation of $D$ is an $(n+2)$-tuple $\left(\Delta, \Pi, X_{u}\right)$ where $X_{u}$ is an $r$-free, $q$-free word such that

(i) if $u$ is odd (i.e. $L_{u}$ is of type $\alpha$ ), then $L_{u}={ }_{H_{2}} \bar{\Delta}^{-1} X_{u} \bar{\Delta}$ and $\bar{\Delta}^{-1} X_{u} \bar{\Delta}$ is stk-reduced;

(ii) if $u$ is even (i.e. $L_{u}$ is of type $\beta$ ), then $L_{u}={ }_{H_{3}} \Pi X_{u} \Pi^{-1}$ and $\Pi X_{u} \Pi^{-1}$ is $s t k$-reduced;

(iii) for every $u, v\left(X_{u}\right)=0$.

A representation $\left(\Delta, \Pi, X_{u}\right)$ of a symmetric word $D$ is called maximal if for every other representation $\left(\Omega, \Phi, Y_{u}\right)$ there exist words $\Theta$ and $\Psi$ (depending on $\left(\Omega, \Phi, Y_{u}\right)$ ) such that $\Delta$ is $\Theta \Omega$ and $\Pi$ is $\Phi \Psi$.

Lемма 35. If $\left(\Delta, \Pi, X_{u}\right)$ is a representation of a symmetric word $D$, then for every $u$ $\sigma\left(X_{u}\right)=0$ and $\left(\exists C_{\varepsilon}\right) X_{u}={ }_{H_{2}} C_{\varepsilon}$.

Proof. It is obvious that $\sigma\left(X_{u}\right)=0$ for all $u$. Suppose that $u$ is odd; then $\bar{\Delta}^{-1} X_{u} \bar{\Delta}_{{ }_{H_{3}} L_{u}}$ whence $\left(\exists C_{\varepsilon}\right) \bar{\Delta}^{-1} X_{u} \bar{\Delta}={ }_{H_{z}} C_{\varepsilon}$. Since $\alpha\left[C_{\varepsilon}\right]$ and $\bar{\Delta}^{-1} X_{u} \bar{\Delta}$ must be stk-parallel, $\alpha\left[C_{\varepsilon}\right]=_{H_{z}}$ $\bar{\Delta}^{-1} x^{\varepsilon\left(2^{c}-1\right)} C_{\varepsilon}^{*} x^{-\varepsilon\left(2^{c}-1\right)} \bar{\Delta}$ where $c=l(\Delta)$. Hence $X_{u}={ }_{H z} x^{\varepsilon\left(2^{c}-1\right)} C_{\varepsilon}^{*} x^{-\varepsilon\left(2^{c}-1\right)}$. Clearly $\sigma\left(C_{\varepsilon}^{*}\right)=0$ and $v\left(C_{\varepsilon}^{* *}\right)=0$ whence $x^{\varepsilon} C_{\varepsilon}^{*} x^{-\varepsilon}={ }_{H_{2}} C_{\varepsilon}^{*}$. Thus $X_{u}={ }_{H_{2}} C_{\varepsilon}^{*}$.

A similar argument applies when $u$ is even.

Let $L$ be any word of $H_{2}$. If $L$ is $\Psi x^{\varepsilon} \varepsilon_{b} L^{\prime}$, then $\Psi_{s} x^{2 \varepsilon} L^{\prime}$ is a primitive $\mu$-reduction of $L$. Also if $L$ is $L^{\prime} s_{b}^{-1} x^{e} \Psi^{-1}$, then $L^{\prime} x^{2 \varepsilon} s_{b}^{-1} \Psi^{-1}$ is a primitive $\mu$-reduction of $L$. We adopt the usual collection of definitions derived from that of a primitive reduction. In particular $\mu[L]$ is the word obtained from $L$ by computing $\mu$-reductions for as long as possible (say always operating on a positive $s$-symbol in preference to a negative $s$-symbol). Then we can effectively ecmpute $\mu[L]$. Intuitively $\mu[L]$ is obtained by pushing to the left end as many positive $s$-symbols as possible and to the right end as many negative $s$-symbols as possible.

LE IM M 36 . If $L$ is stk-reduced, then $\bar{\mu}[L]$ is stk-reduced and $L={ }_{H_{2}} \bar{\mu}[L]$.

Proof. By an obvious induction.

LEMMA 37. Let D be symmetric. Then there is a recursive procedure to compute a maximal representation of $D$.

Proof. Let $D$ have $q$-factors $L_{1}, L_{2}, \ldots, L_{n}$. Compute $\mu\left[L_{1}\right], \mu\left[L_{2}\right], \ldots, \mu\left[L_{n}\right]$. According as $u$ is odd or even, write $\mu\left[L_{u}\right]$ as $\bar{\Delta}_{u-1}^{-1} Y_{u} \bar{\Delta}_{u}$ or $\Pi_{u-1} Y_{u} \Pi_{u}^{-1}$ where $Y_{u}$ does not begin with a positive $s$-symbol nor end with a negative $s$-symbol. (Thus $\bar{\Delta}_{u-1}^{-1}$ or $\Pi_{u-1}$ is the longest

(1) Since $D$ is $q$-alternating, $n$ must in fact be even. 
initial subword of $\mu\left[L_{u}\right]$ which consists entirely of positive $s$-symbols.) Call $(\Delta, \Pi)$ a representative pair if for each $u$, there exist words $\Omega_{u}$ and $\Phi_{u}$ such that

(i) $\Delta_{u}$ is $\Omega_{u} \Delta$ and $\Pi_{u}$ is $\Pi \Phi_{u}$;

(ii) $v\left(\bar{\Omega}_{u-1}^{-1} Y_{u} \bar{\Omega}_{u}\right)=0$ if $u$ is odd and $v\left(\Phi_{u-1} Y_{u} \Phi_{u}^{-1}\right)=0$ if $u$ is even.

Since $D$ is symmetric, taking $\Delta$ to be 1 and $\Pi$ to be 1 , we see that there exist representative pairs. The collection $\left\{\left(\Delta_{u}, \Pi_{u}\right)\right\}$ is finite and so we can recursively determine a representative pair $(\Delta, \Pi)$ such that if $\left(\Delta^{\prime}, \Pi^{\prime}\right)$ is any other representative pair, then $l(\Delta)+l(\Pi)>l\left(\Delta^{\prime}\right)+l\left(\Pi^{\prime}\right)$.

Let $(\Delta, \Pi)$ be this maximal representative pair. Let $X_{u}$ be $\bar{\Omega}_{u-1}^{-1} Y_{u} \bar{\Omega}_{u}$ if $u$ is odd and be $\Phi_{u-1} Y_{u} \Phi_{u}^{-1}$ if $u$ is even, where $\Omega_{u}$ and $\Phi_{u}$ are the words satisfying (i) and (ii) determined by $(\Delta, \Pi)$. We claim that $\left(\Delta, \Pi, X_{u}\right)$ is a maximal representation of $D$. By Lemma 36 it is certainly a representation.

Let $\left(\Omega, \Phi, Z_{u}\right)$ be any other representation. If $u$ is odd, then $\bar{\Delta}^{-1} X_{u} \bar{\Delta}={ }_{H_{2}} \bar{\Omega}^{-1} Z_{u} \bar{\Omega}$ and if $u$ is even, then $\Pi X_{u} \Pi^{-1}={ }_{H_{2}} \Phi Z_{u} \Phi^{-1}$. For each odd $u, \bar{\Delta}^{-1} X_{u} \bar{\Delta}$ and $\bar{\Omega}^{-1} Z_{u} \bar{\Omega}$ must be stk-parallel. Therefore either (a) $\Delta$ is $\Theta \Omega$ for some $\Theta$ or (b) $\Omega$ is $\Theta \Delta$ for some $\Theta$. We want to show that if (b) holds, then $\Theta$ is 1 . Suppose not; then $X_{u}={ }_{H_{z}} \bar{\Theta}^{-1} Z_{u} \bar{\Theta}$ for every odd $u$. Hence, for each odd $u, X_{u}$ and $\bar{\Theta}^{-1} Z_{u} \bar{\Theta}$ are $s t k$-parallel, i.e. $\bar{\Omega}_{u-1}^{-1} Y_{u} \bar{\Omega}_{u}$ and $\bar{\Theta}^{-1} Z_{u} \bar{\Theta}$ are $s t k$ parallel. From the definition of $Y_{u}$ it is clear that $(\Theta \Delta, \Pi)$ is a representative pair. This contradicts the maximality of $(\Delta, \Pi)$. We thus conclude that $\Delta$ is $\Theta \Omega$ for some $\Theta$. In a similar way we see that $\Pi$ is $\Phi \Psi$ for some $\Psi$.

Lemma 38. Let $D$ be a symmetric word with maximal representation $\left(\Delta, \Pi, X_{u}\right)$, $u=1,2, \ldots, n$ and let $P_{1, i}$ be $C_{1}^{(1)} q K_{i} C_{1}^{(2)} K_{i}^{-1} q^{-1} \ldots q K_{i} C_{1}^{(n)} K_{i}^{-1} q^{-1}$. Also let $D_{1}$ be $\bar{F}_{i}^{-1} C_{-1}^{(1)}$ $\bar{F}_{i} q C_{-1}^{(2)} q^{-1} \bar{F}_{i}^{-1} \ldots \bar{F}_{i} q C_{-1}^{(n)} q^{-1}$. If $P_{1, i}$ is $\alpha$-reduced and $D={ }_{H_{1}} P_{1, i}$, then $\bar{F}_{i}^{-1} r_{i}^{-1} D r_{i} \bar{F}_{i}={ }_{G_{0}} D_{1}$ and $D_{1}$ is symmetric. Moreover, there exists a word $\Pi_{1}$ such $\Pi$ is $K_{i} \Pi_{1}$ and $\left(\Delta F_{i}\right.$, $\left.\Pi_{1}, X_{u}\right)$ is a maximal representation of $D_{1}$.

Proof. It is clear that $\bar{F}_{i}^{-1} r_{i}^{-1} D r_{i} \bar{F}_{i}={ }_{G_{0}} D_{1}$. Part of the proof of Lemma 22 shows exactly that $D_{1}$ must be $q$-regular. Obviously $D_{1}$ is $q$-alternating.

Let $M_{u}$ be $\bar{F}_{i}^{-1} C_{-1}^{(u)} \bar{F}_{i}$ or $C_{-1}^{(u)}$ according as $u$ is odd or even. We must verify that for each $u$, (1) $M_{u}$ is stk-reduced, (2) $\sigma\left(M_{u}\right)=0$, (3) $v\left(M_{u}\right)=0$ and (4) ( $\left.\exists C_{\varepsilon}\right) M_{u}={ }_{H_{2}} C_{\varepsilon}$. Let $u$ be odd; each $C_{1}^{(u)}$ is $\alpha$-reduced and if $L_{u}$ is the corresponding $q$-factor of $D$, then $L_{u}={ }_{H_{2}} C_{1}^{(u)}$. From this (1), (2) and (3) are readily verified and (4) also follows easily since $x^{-1} C_{-1}^{(u)} x=_{H_{2}}$ $C_{-1}^{(u)}$ whence $\bar{F}_{j}^{-1} x^{-\left(2^{c}-1\right)} C_{-1}^{(u)} x^{2 c-1} \bar{F}_{i}={ }_{H_{z}} M_{u}$ where $c=l\left(F_{i}\right)$. If $u$ is even, then (1), (2) and (4) are immediate. To prove (3) it suffices to prove that there exists $\Pi_{1}$ such that $\Pi$ is $K_{i} \Pi_{1}$. 
For we know that for each $u, \Pi X_{u} \Pi^{-1}={ }_{H_{2}} L_{u}={ }_{H_{2}} K_{i} C_{1}^{(u)} K_{i}^{-1}$ and $v\left(X_{u}\right)=0$. Now $\Pi X_{u} \Pi^{-1}$ and $K_{i} \gamma_{1}^{(u)} K_{i}^{-1}$ must be $s t k$-parallel. We must show that it is impossible that $K_{i}$ is $\Pi_{1}$ with $\Pi_{1}$ distinct from 1 . Therefore suppose this occurs; then for each $u, X_{u}={ }_{H_{2}} \Pi_{1} C_{1}^{(u)} \Pi_{1}^{-1}$. By Lenima 35, $\left(\exists C_{1}^{*}\right) X_{u}={ }_{H_{2}} C_{1}^{*}$. Hence, if $c=l\left(\Pi_{1}\right), X_{u}={ }_{H_{2}} \Pi_{1} x^{2^{c}-1} C_{1}^{(u)} x^{-\left(2^{c}-1\right)} \Pi_{1}^{-1}$ whence it follows that $v\left(C_{1}^{(u)}\right)=0$.

Let $Y_{u}$ be $X_{u}$ if $u$ is odd and $C_{1}^{(u)}$ if $u$ is even. Then $\left(\Delta, K_{i}, Y_{u}\right)$ is a representation of $D$. This contradicts the maximality of $\left(\Delta, \Pi, X_{u}\right)$. Hence $\Pi$ must be $K_{i} \Pi_{1}$ whence $\nu\left(C_{-1}^{(u)}\right)=0$.

In the process we have also proved our second assertion. It remains to verify that $\left(\Delta F_{i}, \Pi_{1}, X_{u}\right)$ is a maximal representation. It is easy to show that for every $u, \sigma\left(C_{1}^{(u)}\right)=0$ and $\nu\left(C_{: .}^{(u)}\right)=0$ whence $C_{-1}^{(u)}={ }_{H_{\mathrm{a}}} C_{1}^{(u)}$. Using this last fact, it may be readily be shown that $\left(\Delta F_{i}, \Pi_{1}, X_{u}\right)$ is a representation. Suppose that $\left(\Omega, \Phi, Y_{u}\right)$ is a maximal representation. Then $\Omega$ is $\Theta \Delta F_{i}$ and $\Phi$ is $\Pi_{1} \Psi$. We want to show that $\Theta$ is 1 and $\Psi$ is 1 . Assume that $\Theta$ is distinct from 1 ; if $u$ is odd, then $\bar{\Omega}^{-1} Y_{u} \bar{\Omega}={ }_{H_{z}} \bar{F}_{i}^{-1} \bar{\Delta}^{-1} X_{u} \bar{\Delta} \bar{F}_{i}$ whence $\bar{\Delta}^{-1} \bar{\Theta}-1 Y_{u} \bar{\Theta} \bar{\Delta}={ }_{H_{z}}$ $\bar{\Delta}^{-1} X_{u} \bar{\Delta}$. Let $Z_{u}$ be $Y_{u}$ if $u$ is odd and $X_{u}$ if $u$ is even. Then $\left(\Theta \Delta, \Pi, Z_{u}\right)$ is a representation of $j$. This is impossible. In a similar way we can prove that $\Psi$ is 1 .

Let $A$ be given; we can associate with $A$, in a natural way, a sequence of operations of $\mathfrak{I}_{*}$. The association is defined as follows:

(1) if $A$ is 1 , the corresponding sequence is empty;

(2) if $A$ is $r_{i} \bar{F}_{i} A^{\prime}$, the corresponding sequence consists of $q K_{i} \rightarrow F_{i} q$ followed by the sequence defined by $A^{\prime}$;

(3) if $A$ is $\bar{F}_{i}^{-1} r_{i}^{-1} A^{\prime}$, the corresponding sequence consists of $F_{i} q \rightarrow q K_{i}$ followed by the sequence defined by $A^{\prime}$.

A word $A$ is called proof-inducing if there exist words $\Delta q \Pi$ and $\Omega q \Phi$ of $\mathfrak{I}_{*}$ such that $\Delta q \Pi$ can be transformed into $\Omega q \Phi$ by the sequence of operations defined by $A$. In such circumstances we shall say that $A$ specifies a proof that $\Delta q \Pi=\mathfrak{I}_{*} \Omega q \Phi$.

TH:OREM 8. Let $D$ and $E$ be q-parallel and symmetric words with maximal representations $\left(\Delta, \Pi, X_{u}\right)$ and $\left(\Omega, \Phi, Y_{u}\right)$ respectively. If $A$ is $\alpha$-reduced, then $A^{-1} D A={ }_{G_{0}} E$ if and only if $A$ specifies a proof that $\Delta q \Pi=\mathfrak{x}_{*} \Omega q \Phi$ and, for each $u, X_{u}={ }_{H_{2}} Y_{u}$.

Proof. Suppose that $A^{-1} D A={ }_{G_{0}} E$; we proceed by induction w.r.t. $l_{r}(A)$. If $l_{r}(A)=0$, then $D={ }_{G_{\theta}} E$ whence it follows that $\left(\Omega, \Phi, Y_{u}\right)$ is a representation for $D$ and $\left(\Delta, \Pi, X_{u}\right)$ is a representation for $E$. This implies that $\Delta$ is $\Omega, \Pi$ is $\Phi$ and that $X_{u}={ }_{H_{z}} Y_{u}$.

Let $l_{r}(A)>0$ and suppose that $A$ is $r_{i} \bar{F}_{i} A^{\prime}$. Then $\left(\exists P_{1, i}\right) D={ }_{H_{1}} P_{1, i}$. If $D$ is $L_{1} q L_{2} q^{-1} \ldots$ $q L_{n} q^{-1}$, then $\alpha\left[P_{1, i}\right]$ must be $C_{1}^{(1)} q K_{i} C_{1}^{(2)} K_{i}^{-1} q^{-1} \ldots q K_{i} C_{1}^{(n)} K_{i}^{-1} q^{-1}$. This means that the 10-692905 Acta mathematica 122. Imprimé le 21 mars 1969 
hypotheses of Lemma 38 are satisfied by $D$ and $\alpha\left[P_{1},{ }_{i}\right]$. With $D_{1}$ as in Lemma 38, we have $\bar{F}_{i}^{-1} r_{i}^{-1} D r_{i} \bar{F}_{i=G_{0}} D_{1}$ and $A^{\prime-1} D_{1} A^{\prime}={ }_{G 0} E$. Since $D_{1}$ has maximal representation $\left(\Delta F_{i}, \Pi_{1}\right.$, $\left.X_{u}\right)$, by the inductive hypothesis $A^{\prime}$ specifies a proof that $\Delta F_{i} q \Pi_{1}=\mathfrak{I}_{*} \Omega q \Phi$ and, for each $u, X_{u}={ }_{H_{2}} Y_{u}$. But $\Delta q \Pi$ is $\Delta q K_{t} \Pi_{1}$ whence it follows that $A$ specifies a proof that $\Delta q \Pi=\mathfrak{T}_{*} \Omega q \Phi$.

If $A$ is $\bar{F}_{i}^{-1} r_{i}^{-1} A^{\prime}$, the argument required is dual to the above (and employs the lemma, which we have left unstated, dual to Lemma 38).

Conversely suppose that $A$ specifies a proof that $\Delta q \Pi=\tau_{\tau_{*}} \Omega q \Phi$ and that $X_{u}={ }_{H_{2}} Y_{u}$ for all $u$. Again we proceed by induction w.r.t. $l_{r}(A)$. If $l_{r}(A)=0$, then $\Delta$ is $\Omega$ and $\Pi$ is $\Phi$ whence it follows that $D={ }_{G \theta} E$.

Let $l_{r}(A)>0$ and suppose that $A$ is $r_{i} \bar{F}_{i} A^{\prime}$. Then $\Delta q \Pi$ is $\Delta q K_{i} \Pi_{1}$ and is transformed to $\Delta F_{i} q \Pi_{1}$. Moreover $A^{\prime}$ specifies a proof that $\Delta F_{i} q \Pi_{1}=\tau_{*} \Omega q \Phi$. For each odd $u$, $\left(\exists C_{\varepsilon}\right) \bar{\Delta}^{-1} X_{u} \bar{\Delta}={ }_{H_{s}} C_{\varepsilon}$ with $\sigma\left(C_{\varepsilon}\right)=0$ and $\nu\left(C_{\varepsilon}\right)=0$, whence $\bar{F}_{i}^{-1} r_{i}^{-1} \bar{\Delta}^{-1} X_{u} \bar{\Delta} r_{i} \bar{F}_{i}={ }_{G_{0}} \bar{F}_{i}^{-1} \bar{\Delta}^{-1}$ $X_{u} \bar{\Delta} \bar{F}_{i}$. For each even $u, \sigma\left(X_{u}\right)=0$ and $\nu\left(X_{u}\right)=0$ and $\left(\exists C_{\varepsilon}\right) X_{u}={ }_{H_{z}} C_{\varepsilon}$. Hence $\left(\exists C_{\varepsilon}^{*}\right)$ $\Pi_{1} X_{u} \Pi_{1}^{-1}={ }_{H_{z}} C_{\varepsilon}^{*}$ with $\sigma\left(C_{\varepsilon}^{*}\right)=0$ and $\nu\left(C_{\varepsilon}^{*}\right)=0$ and this implies that $r_{i}^{-1} K_{i}^{-1} \Pi X_{u} \Pi^{-1} K_{i} r_{i}$ $={ }_{G_{0}} \Pi_{1} X_{u} \Pi_{1}^{-1}$. Let $L_{u}^{\prime}$ be $\bar{F}_{i}^{-1} \bar{\Delta}^{-1} X_{u} \bar{\Delta} \bar{F}_{i}$ if $u$ is odd and be $\Pi_{1} X_{u} \Pi_{1}^{-1}$ if $u$ is even. If $D_{1}$ is $L_{1}^{\prime} q L_{2}^{\prime} q^{-1} \ldots q L_{n}^{\prime} q^{-1}$, then $D_{1}$ is easily seen to be symmetric with maximal representation $\left(\Delta F_{i}, \Pi_{1}, X_{u}\right)$. By the hypothesis of induction, $A^{\prime-1} D_{1} A^{\prime}={ }_{G_{0}} E$. Since $\bar{F}_{i}^{-1} r_{i}^{-1} D r_{i} \bar{F}_{i}$ ${ }_{G 0} D_{1}$, the theorem follows.

The argument for the case in which $A$ is $\bar{F}_{i}^{-1} r_{i}^{-1} A^{\prime}$ is dual to the above.

REDUCTION V. (? $D, E q$-regular $)(\exists A) A^{-1} D A={ }_{G_{0}} E \longrightarrow(? \Delta q \Pi, \Omega q \Phi) \Delta q \Pi=\mathfrak{x}_{*} \Omega q \Phi$.

Proof. Let $D$ and $E$ be given. If they are not $q$-parallel then they are not conjugate by a word $A$. So suppose they are $q$-parallel; by Lemma 20 we may assume that they are factor-reduced. Also it is clear that we need only consider whether or not there exists an $\alpha$-reduced $A$ which conjugates $D$ into $E$.

The content of Theorems 4-7 is that if either $D$ or $E$ is not symmetric, then there is an effectively computable upper bound (namely 3 ) on the number of $r$-symbols occurring in any $\alpha$-reduced $A$ which conjugates $D$ into $E$. Now $(? L)\left(\exists C_{\varepsilon}\right) L={ }_{H_{8}} C_{\varepsilon}$ is recursively solvable (see Solution 4 ) and hence there is a recursive prodedure to determine whether or not $D$ and $E$ are symmetric. It therefore follows that if $D$ or $E$ is not symmetric, then (? $D, E q$-regular) $(\exists A) A^{-1} D A={ }_{G_{0}} E$ is reducible to the word problem for $G_{0}$ and is thus solvable.

Now suppose that $D$ and $E$ are symmetric. By Lemma 37, we can recursively compute maximal representations $\left(\Delta, \Pi, X_{u}\right)$ and $\left(\Omega, \Phi, Y_{u}\right)$ for $D$ and $E$. By Theorem 8 , 
( $\exists A) A^{-1} D A={ }_{G_{0}} E$ if and only if $\Delta q \Pi={ }_{\mathfrak{I}_{*}} \Omega q \Phi$ and, for each $u, X_{u}={ }_{H_{2}} Y_{u}$. Since $H_{2}$ has a solvable word problem, this completes the logical reduction.

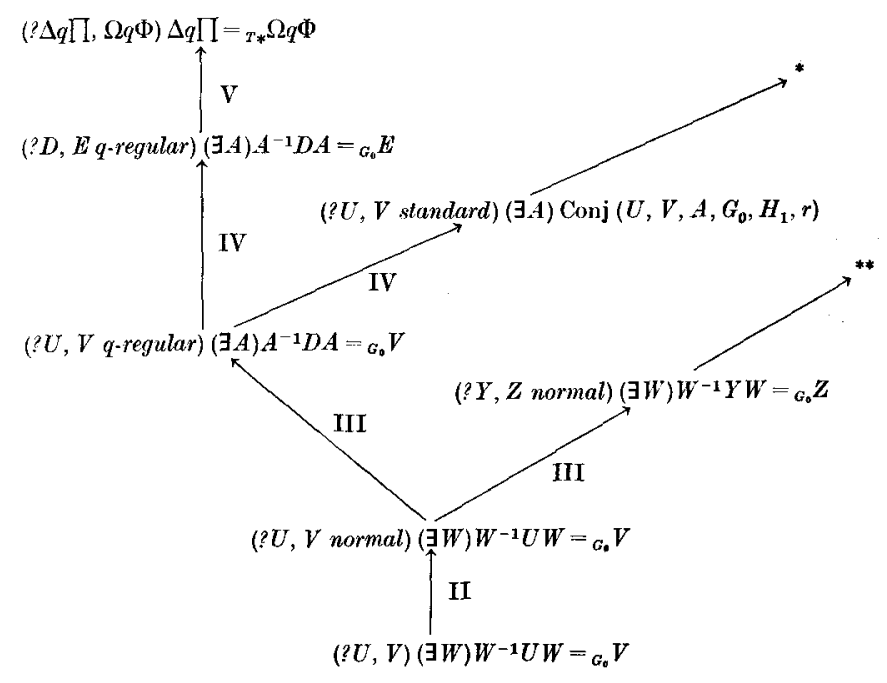

* Continued on Diagram C

** Continued on Diagram D

Diagram B

$\S 4$. In this section we provide a recursive solution of (? $U, V$ standard) $(\exists A) \operatorname{Conj}(U, V$, $\left.A, G_{0}, H_{1}, r\right)$. For brevity we shall write $\operatorname{Conj}(U, V, A, r)$ rather than $\operatorname{Conj}(U, V, A$, $\left.G_{0}, H_{1}, r\right)$.

Let $U$ be a standard word. If $U$ is $U_{1} r_{i}^{-1} D$ and $\left(\exists P_{1, i}\right)\left[D={ }_{H_{1}} P_{1, i} K_{i}^{-1} q^{-1}\right.$ and $P_{1, i} K_{i}^{-1} q^{-1}$ is $\alpha$-reduced], then the first z-reduction of $U$ is $\bar{F}_{i}^{-1} r_{i}^{-1} U_{1} P_{-1, i} q^{-1}$. If $U$ is $U_{1} r_{i} D$ and $\left(\exists P_{-1, i}\right)\left[D={ }_{H_{1}} P_{-1, i} q^{-1}\right.$ and $P_{-1, i} q^{-1} \bar{F}_{i}^{-1}$ is $\alpha$-reduced $]$, then the first z-reduction of $U$ is $r_{i} \bar{F}_{i} U_{1} P_{1, i} K_{i}^{-1} q^{-1}$. If neither of the above is applicable, then the first $z$-reduction of $U$ is to be $U$. We shall write $z_{1}[U]$ for the first $z$-reduction of $U$. The $n$-th z-reduction, $z_{n}[U]$, is $z_{1}\left[z_{n-1}[U]\right]$. Also let $z_{0}[U]$ be $U .\left(^{(1)}\right.$

Lе мг м 39. Let $U$ be any standard word. Then (i) for every $n,(\exists A) \operatorname{Conj}\left(U, z_{n}[U], A, r\right)$; (ii) if $(\exists i \imath)(\exists A) \operatorname{Conj}\left(z_{n}[U], V, A, r\right)$, then $(\exists A) \operatorname{Conj}(U, V, A, r)$.

Proof. Both assertions follow, by an inductive argument, from the definition of $z_{n}[U]$.

(1) The purpose of the definition of $z_{n}[U]$ is to break down the conjugation of $U$ into $V$ into stages. If Conj $(U, V, A, r)$ holds and $A$ is $A_{i_{1}}^{e_{1}} A_{i_{3}}^{e_{2}} \ldots A_{i_{n}}^{e_{n}}$ then $z_{k}[U]$ is the word obtained from $U$ by conjugating by $A_{i_{1}}^{e_{1}} A_{i_{2}}^{e_{2}} \ldots A_{i_{k}}^{e_{k}}$. Also we shall say that $z_{1}[U]$ is obtained trivially from $U$ if $z_{1}[U]$ is $U$. 
LEMMA 40. Let $U$ be a standard word. For each $n, z_{n}[U]$ is standard and q-parallel to $U$.

Proof. It suffices to verify the lemma when $n=1$. We must show firstly that $z_{1}[U]$ is $r$-contracted, $q$-contracted and $t k$-contracted. Supposing the contrary leads routinely to a contradiction. By definition $z_{1}[U]$ terminates in $q^{-1}$ and thus is standard. The $q$-parallelism follows from the fact that $P_{1, i} K_{i}^{-1} q^{-1}$ or $P_{-1, i} q^{-1} \bar{F}_{i}^{-1}$, as is appropriate, is $\alpha$-reduced.

LEMMA 41. Let $U$ and $V$ be standard words. Then $(\exists A)$ Conj $(U, V, A, r)$ if and only if $(\exists n)(\exists A)\left[n<l_{r}(A), z_{n}[U]\right.$ is r-parallel to $V$ and $\left.\operatorname{Conj}\left(z_{n}[U], V, A, r\right)\right]$.

Proof. Suppose that Conj $(U, V, A, r)$ holds; let $m=l_{r}(A)$. Let $R_{j}$ be $\pi_{r}\left[z_{j}[U]\right]$, $j=0,1, \ldots, m$. From the definitions of $z$-reduction and Conj, it is clear that if $R_{j}$ is $R_{j}^{\prime} r_{i}^{\varepsilon}$, then $R_{j+1}$ is $r_{i}^{\varepsilon} R_{j}^{\prime}$. If $m<l_{r}(U)$, take $n=m$. If $m \geqslant l_{r}(U)$, the collection of words $\left\{R_{j}\right\}$, $j=0,1, \ldots, l_{r}(U)-1$, consists of all possible cyclic permutations of $\pi_{r}[V]$. This proves one half of the double implication.

The converse is immediate from Lemma 39.

REDUCTION VI. (? $U, V$ standard $)(\exists A) \operatorname{Conj}(U, V, A, r) \longrightarrow(? U, V$ standard, $r$-parallel $)(\exists A) \operatorname{Conj}(U, V, A, r)$ and $(? D)\left(\exists P_{\varepsilon, i}\right) D={ }_{H_{1}} P_{\varepsilon, i}$.

Proof. By Lemma 41, it suffices to show that the problem of computing $z_{n}[U]$ is reducible to $(? D)\left(\exists P_{\varepsilon, i}\right) D={ }_{H_{1}} P_{\varepsilon, i}$. In one case we must decide for a given $q$-reduced $D$ of form $D^{\prime} q^{-1}$ whether or not $D={ }_{H_{1}} P_{1, i} K_{i}^{-1} q^{-1}$ with $P_{1, i} K_{i}^{-1} q^{-1} \alpha$-reduced. It is easy to see that this occurs if and only if $\left(\exists P_{1, i}\right) D={ }_{H_{1}} P_{1, i}$. In a similar way, $U_{1} r_{i} D$ will produce $z_{1}[U]$ non-trivially if and only if $\left(\exists P_{-1, i}\right) D \bar{F}_{\xi}^{-1}={ }_{H_{1}} P_{-1, i}$.

LЕмм А 42. Let $W_{1}$ and $W_{2}$ be two non-empty words. If $W_{1} W_{2}$ coincides with $W_{2} W_{1}$, then there exists a word $W_{0}$ such that $W_{1}$ is $W_{0}^{m_{1}}$ and $W_{2}$ is $W_{0}^{m_{2}}$ for some natural numbers $m_{1}$ and $m_{2}$.

Proof. This lemma is proved by Lyndon and Schutzenberger in [14].

LEMMA 43. Let $W_{1}$ and $W_{2}$ be two non-empty words. If, for some natural number $m$, $\left(W_{1} W_{2}\right)^{m}$ coincides with $\left(W_{2} W_{1}\right)^{m}$, then there exists a word $W_{0}$ and natural numbers $m_{1}$ and $m_{2}$ such that $W_{1}$ is $W_{0}^{m_{1}}$ and $W_{2}$ is $W_{0}^{m_{3}}$.

Proof. If $\left(W_{1} W_{2}\right)^{m}$ coincides with $\left(W_{2} W_{1}\right)^{m}$, then $W_{1} W_{2}$ coincides with $W_{2} W_{1}$ and therefore Lemma 42 is applicable.

Let $U$ be any word and let $R$ be the $r$-projection of $U$. A word $R_{0}$ is called an $r$-divisor of $U$ if there exists a positive integer $m$ such that $R_{0}^{m}$ coincides with $R$. Clearly for any word $U$, there is a unique $r$-divisor of $U$ which is minimal in length. We shall call this unique $r$-divisor the minimal $r$-divisor of $U$ and write $R_{U}$ for it. 
LIM MA 44. Let $U$ and $V$ be standard, $r$-parallel words with (common) minimal $r$-divisor $R_{U}$. If Conj $(U, V, A, r)$ holds and $l_{r}(A)<l\left(R_{U}\right)$, then $l_{r}(A)=0$.

Proof. Let $\pi_{r}(A)$ be $R_{1}^{-1}$. Then there must exist $R_{2}$ such that $R_{U}$ is $R_{2} R_{1}$. Suppose that $l\left(R_{1}\right) \neq 0$; then $r\left[A^{-1} U A\right]$ must have $r$-projection $R_{1} R_{U}^{m-1} R_{2}$ where $R_{U}^{m}$ is $\pi_{r}(U)$. Since $V$ and $r\left[A^{-1} U A\right]$ are $r$-parallel, $\left(R_{2} R_{1}\right)^{m}$ is $\left(R_{1} R_{2}\right)^{m}$. By Lemma 43 , the minimality of $R_{U}$ is contradicted.

Lхмма 45. Let $U$ and $V$ be standard, r-parallel words with minimal $r$-divisor $R_{U}$ and let $A_{U}$ have r-projection $R_{U}^{-1}$. If $\operatorname{Conj}(U, V, A, r)$ holds, then there exists a natural number $m$ such that $A$ is $A_{U}^{m}$.

Proof. We proceed by induction w.r.t. $l_{r}(A)$. If $l_{r}(A)=0$, take $m=0$. If $l_{r}(A)>0$, then it follows from Lemma 44 that $l_{r}(A) \geqslant l\left(R_{v}\right)$. We may thus write $A$ as $A^{*} A^{\prime}$ where $l_{r}\left(A^{*}\right)=$ $l\left(R_{U}\right)$ a:ad clearly $\pi_{r}\left[A^{*}\right]$ is $R_{U}^{-1}$. Also if $n^{*}=l_{r}\left(A^{*}\right)$, then Conj $\left(z_{n^{*}}[U], V, A^{\prime}, r\right)$ must hold. We may therefore apply the inductive hypothesis to $z_{n^{*}}[U]$ and $V$ to obtain that $A^{\prime}$ is $A_{U}^{m^{\prime}}$. Then $A$ is $A_{U}^{m^{\prime}+1}$.

LF M A 46. Let $U$ be standard with minimal $r$-divisor $R_{U}$ and r-projection $R_{U}^{s}, s \geqslant 1$, and let $z_{n}[U]$ be r-parallel to $U$. If $A_{U}$ has r-projection $R_{U}^{-1}$ and there exists $m \geqslant s$ such that Conj $\left(U, z_{n}[U], A_{U}^{m}, r\right)$, then there exists an $r$-free, $q$-regular word $D$ such that $z_{n}[U]$ is $A_{U}^{-s} D$.

Proof. If $n_{1}=l_{r}(U)$, then $\operatorname{Conj}\left(U, z_{n_{1}}[U], A_{U}^{s}, r\right)$ must hold. It is clear from the definitions of $z$-reduction and Conj that $z_{n_{1}}[U]$ has the form $A_{U}^{-s} D_{1}$, where $D_{1}$ is $r$-free. $\left(D_{1}\right.$ is obtained by $r$-reducing, in a particular way, $U A_{U}^{s}$.) If $n_{2}=n_{1}+l_{r}\left(A_{U}\right)$, then $z_{n_{2}}[U]$ must have the form $A_{U}^{-s} D_{2}$ where $D_{2}$ is obtained by $r$-reducing $A_{U}^{-s} D_{1} A_{U}^{s}$ (plus perhaps some cancellation of inverse pairs of generating symbols). The lemma clearly follows by an inductive argument once we note that since every $z_{n}[U]$ is standard, the appropriate $D$ will be $q$ regular.

LEMMA 47. Let $V$ be standard and let $A$ be such that $r[A V]$ is the $q$-free word $E$. Then E is q-regular.

Prcof. It is easy to verify that $A V$ is $q$-contracted and $t k$-contracted. The process of computing primitive $r$-reductions does not disturb $q$ - and $t k$-contractedness (see part (ii) of Theorem 2). Hence $E$ is $q$ - and $t k$-contracted. Moreover, in the computation of $r[A V]$ the final $q$-symbol of $V$ clearly remains undisturbed and thus $E$ is $q$-regular.

L. $\mathrm{MMA}$ 48. Let $A, D$ and $E$ be such that $A^{-s} D$ and $A^{-s} E$ are standard. If there exists $m>0$ such that Conj $\left(A^{-s} D, A^{-s} E, A^{m}, r\right)$ holds, then $A$ is r-contracted. 
Proof. If $s>1, A$ must be $r$-contracted since $A^{-s} D$ is $r$-reduced. So let $s=1$; since $A$ must be $r$-reduced $A$ will not be $r$-contracted only if $A$ is of form $r_{i} \bar{F}_{i} A^{\prime} \bar{F}_{i}^{-1} r_{i}^{-1}$ or $\bar{F}_{i}^{-1} r_{i}^{-1} A^{\prime} r_{i} \bar{F}_{i}$. Suppose the former occurs; since Conj $\left(A^{-1} D, A^{-1} E, A^{m}, r\right)$ holds with $m>0,\left({ } P_{1, i}\right) D={ }_{H_{1}} P_{1, i}$. But this implies that $A^{-1} D$ is not $r$-contracted which is a contradiction. A similar argument holds for the second case.

REDUCTION VII (i). (? $U, V$ standard, r-parallel) $(\exists A) \operatorname{Conj}(U, V, A, r) \longrightarrow(? D, E$, $A, s ; D, E$ q-regular, $A$ r-contracted, $s \geqslant 1)(\exists m>0) \operatorname{Conj}\left(A^{-s} D, A^{-s} E, A^{m}, r\right)$ and $(? D)\left(\exists P_{\varepsilon, i}\right)$ $D={ }_{H_{1}} P_{\varepsilon, i}$.

Proof. Let $n_{0}=l_{r}(U)$; compute $z_{k}[U], k=1,2, \ldots, n_{0}-1$ (we proved in Reduction VI that the problem of doing this is reducible to $\left.(? D)\left(\exists P_{\varepsilon, i}\right) D={ }_{H_{2}} P_{\varepsilon, i}\right)$. By the solvability of the word problem we can determine whether or not there exists $k$ in this range such that $V={ }_{G \bullet} z_{k}[U]$. If this is so, then certainly $(\exists A) \operatorname{Conj}(U, V, A, r)$ holds. If this is not the case, then Conj $(U, V, A, r)$ holds only if $l_{r}(A) \geqslant n_{0}$.

Suppose then that no $k$ in the above range is such that $V={ }_{G_{0}} z_{k}[U]$. Compute $z_{n_{0-1}}[U]$; if $z_{n_{0}}[U]$ is obtained trivially from $z_{n_{0}-1}[U]$, then it is clear that there does not exist a word $A$ such that Conj ( $U, V, A, r)$ holds. So assume that $z_{n_{0}}[U]$ has been obtained nontrivially from $z_{n_{0}-1}[U]$. In these circumstances, $z_{n_{0}}[U]$ must be $r$-parallel to $U$. If $R_{U}$ and $A_{U}$ are defined as in Lemma 45, then $z_{n_{0}}[U]$ must have the form $A_{U}^{-s} D$ where $D$ is $q$ regular. By Lemma 39 , it suffices to determine whether or not there exists $A$ such that Conj ( $\left.A_{U}^{-s} D, V, A, r\right)$ holds.

Since $V$ and $A_{U}^{-s} D$ are $r$-parallel, if $(\exists A) \operatorname{Conj}\left(A_{U}^{-s} D, V, A, r\right)$ holds, then there exist natural numbers $m$ and $n$ and a word $D_{1}$ such that $A$ is $A_{U}^{m}, V={ }_{G_{0}} z_{n}[U]$ and $z_{n}[U]$ has the form $A_{U}^{-8} D_{1}$ with $D_{1} r$-free. Compute $r\left[A_{U}^{s} V\right]$; if it is not $r$-free then $(\exists A) \operatorname{Conj}\left(A_{U}^{-s} D, V, A, r\right)$ fails. Thus we may assume that $r\left[A_{U}^{s}\right]$ is the $r$-free word $E$. By Lemma $47, E$ is $q$-regular. To solve our problem it now suffices to determine of the words $D, E$ and $A_{U}$ whether or not there exists $m \geqslant 0$ such that Conj $\left(A_{U}^{-s} D, A_{U}^{-s} E, A_{U}^{m}, r\right)$ holds. Since $(\exists m \geqslant 0)$ Conj $\left(A_{U}^{-s} D\right.$, $\left.A_{U}^{-s} E, A_{U}^{m}, r\right)$ if and only if $(\exists m>0) \operatorname{Conj}\left(A_{U}^{-s} D, A_{U}^{-s} E, A_{U}^{m}, r\right)$ or $A_{U}^{-s} D={ }_{G_{0}} A_{U}^{-s} E$, the logical reduction is almost complete. It remains only to show that $A_{U}$ is in fact $r$-contracted. Since $A_{U}^{-s} E={ }_{G_{\vartheta}} V$ and $V$ is standard, it follows that $A_{U}^{-s} E$ is standard. Now apply Lemma 48.

\section{REDUCTION VII (ii).}

$(? D, E, A, s ; D, E q$-regular, $A$ r-contracted, $s \geqslant 1)(\exists m>0) \operatorname{Conj}\left(A^{-s} D, A^{-s} E, A^{m}, r\right) \longrightarrow$ $(? D, E, A ; D, E q$-regular, $A$ r-contracted $)(\exists m) A^{-m} D A^{m}={ }_{G_{0}} E$.

Proof. The reduction is a consequence of the fact that $(\exists m>0) \operatorname{Conj}\left(A^{-s} D, A^{-s} E\right.$, $\left.A^{m}, r\right)$ if and only if $(\exists m>0) A^{-m} D A^{m}={ }_{G_{0}} E$. This equivalence is easily verified. 


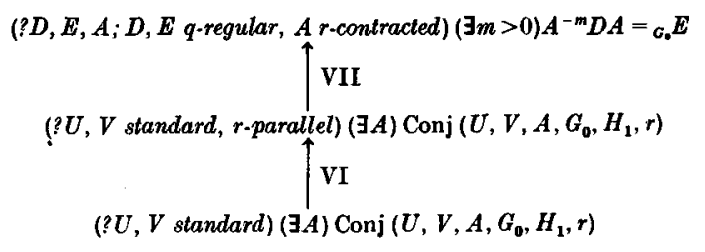

Diagram C

LF мм 49. Let $A$ be proof-inducing. Then there exist words $\Theta, \Psi, \Lambda$ and $\Xi$ such that (i) A specifies a proof that $\Theta q \Psi=\mathfrak{I}_{*} \Lambda q \Xi$; (ii) if $A$ specifies a proof that $\Delta q \Pi=\mathfrak{T}_{*} \Omega q \Phi$, then there exist words $\Delta^{*}$ and $\Pi^{*}$ such that $\Delta$ is $\Delta^{*} \Theta, \Pi$ is $\Psi^{*} \Pi^{*}, \Omega$ is $\Delta^{*} \Lambda$ and $\Phi$ is $\Xi \Pi^{*}$. Moreover, there is a recursive procedure to compute $\Theta, \Psi, \Lambda$ and $\Xi$ from $A .\left({ }^{1}\right)$

Proof. We proceed by induction w.r.t. $l_{r}(A)$. If $l_{z}(A)=0$, take $\Theta, \Psi, \Lambda$ and $\Xi$ to be 1 . Supposis that $l_{r}(A)>0$ and let $A$ be $A_{i} A^{\prime}$. Then the first operation is $q K_{i} \rightarrow F_{i} q$. Since $A$ is proof-inducing, $A^{\prime}$ must be proof-inducing. Let $\Theta^{\prime}, \Psi^{\prime \prime}, \Lambda^{\prime}$ and $\Xi^{\prime}$ be determined, according to the inductive hypothesis, from $A^{\prime}$. Now either $l\left(F_{i}\right) \leqslant l\left(\Theta^{\prime}\right)$ or $l\left(F_{i}\right)>l\left(\Theta^{\prime}\right)$. In the former case we claim that there exists $\Theta^{\sim}$ such that $\Theta^{\prime}$ is $\Theta^{\sim} F_{i}$. Since $A$ is proof-inducing, there exist words $\Delta q \Pi$ and $\Omega q \Phi$ such that $A$ specifies a proof that $\Delta q \Pi={ }_{\mathfrak{x}_{*}} \Omega q \Phi$. Then there exists $\Pi_{1}$ such that $\Pi$ is $K_{i} \Pi_{1}$ and $A^{\prime}$ specifies a proof that $\Delta F_{i} q \Pi_{1}=\mathfrak{x}_{*} \Omega q \Phi$. By the inductive hypothesis, there exists a word $\Delta^{\prime *}$ such that $\Delta F_{i}$ is $\Delta^{\prime *} \Theta^{\prime}$. The existence of $\Theta^{\sim}$ follows from this. Part (i) is then proved by taking $\Theta$ to be $\Theta^{\sim}, \Psi$ to be $K_{i} \Psi^{\prime \prime}, \Lambda$ to be $\Lambda^{\prime}$ and $\Xi$ to be $\Xi^{\prime}$. To prove part (ii), we take $\Delta^{*}$ to be $\Delta^{\prime *}$ and $\Pi^{*}$ to be $\Pi^{\prime *}$ where $\Delta^{\prime *}$ and $\Pi^{\prime *}$ correspond to $A^{\prime}$ according to the inductive hypothesis.

In the case when $l\left(F_{i}\right)>l\left(\Theta^{\prime}\right)$, it is easy to verify that there exists $\Theta$ - such that $F_{i}$ is $\Theta^{\sim} \Theta^{\prime}$. To prove (i), we take $\Theta$ to be $1, \Psi^{*}$ to be $K_{i} \Psi^{\prime}, \Lambda$ to be $\Theta^{-} \Lambda^{\prime}$ and $\Xi$ to be $\Xi^{\prime}$. To prove (ii), we take $\Delta^{*}$ to be $\Delta^{* *}$ and $\Pi^{*}$ to be $\Pi^{\prime *}$. The whole computation is clearly recursive.

If $A$ is $A_{i}^{-1} A^{\prime}$, the argument is similar.

So.tuTION 8. "(?A) Is A proof-inducing" is recursively solvable.

Proof. This is proved by an inductive argument similar to the proof of Lemma 49 .

LEMMA 50. Let $\Delta_{0} q \Pi_{0}=\mathfrak{I}_{*} \Delta_{1} q \Pi_{1}=\mathfrak{I}_{*} \ldots=\mathfrak{I}_{*} \Delta_{m} q \Pi_{m}$ where each proof that $\Delta_{j} q \Pi_{j}=\mathfrak{I}_{*}$ $\Delta_{j+1} q \Pi_{j+1}$ is specified by a common fixed word $A$. Let $\Theta, \Psi, \Lambda$ and $\Xi$ be determined by $A$ as per Lemma 49. Then

( $\left.{ }^{1}\right)$ 'The essential content of the lemma is that common to every proof specified by $A$, there is a central cire within which the operation rules are applied. 
(i) if $l(\Lambda)<l(\Theta)$, then $m \leqslant l\left(\Delta_{0}\right)$;

(ii) if $l(\Xi)<l(\Psi)$, then $m \leqslant l\left(\Pi_{0}\right)$;

(iii) if $l(\Lambda)=l(\Theta)$ and $l(\Xi)=l(\Psi)$, then $\Delta_{0}, \Delta_{1}, \ldots, \Delta_{m}$ coincide and $\Pi_{0}, \Pi_{1}, \ldots, \Pi_{m}$ coincide;

(iv) if $l(\Lambda)>l(\Theta)$, then $m \leqslant l\left(\Delta_{m}\right)$;

(v) if $l(\Xi)>l(\Psi)$, then $m \leqslant l\left(\Pi_{m}\right)$.

Proof. (i) It suffices to show that, for every $j, l\left(\Delta_{j}\right)>l\left(\Delta_{j+1}\right)$. By Lemma 49, there exists $\Delta_{j}^{*}$ such that $\Delta_{j}$ is $\Delta_{j}^{*} \Theta$ and $\Delta_{j+1}$ is $\Delta_{j}^{*} \Lambda$. This implies that $l\left(\Delta_{j}\right)>l\left(\Delta_{j+1}\right)$. (ii) This is similar to (i). (iii) By Lemma 49 , there exist $\Delta_{j}^{*}$ and $\Delta_{j+1}^{*}$ such that $\Delta_{j+1}$ is $\Delta_{j}^{*} \Lambda$ and also $\Delta_{j+1}^{*} \Theta$. Since $l(\Lambda)=l(\Theta), \Delta_{j}^{*}$ coincides with $\Delta_{j+1}^{*}$. Also by Lemma $49, \Delta_{j}$ is $\Delta_{j}^{*} \Theta$ whence it follows that $\Delta_{j}$ and $\Delta_{j+1}$ must coincide. A similar argument shows that $\Pi_{j}$ and $\Pi_{j+1}$ coincide. (iv) It is easy to prove that, for every $j, l\left(\Delta_{j}\right)<l\left(\Delta_{j+1}\right)$. This suffices. ( $v$ ) This is similar to (iv).

Soldton 9. (? $D, E, A ; D, E$-regular, $A$ r-contracted $)(\exists m>0) A^{-m} D A^{m}={ }_{G_{0}} E$ is recursively solvable.

Proof. By Theorems 4-7, if either $D$ or $E$ is not symmetric, then $A^{-m} D A^{m}=c_{0} E$ implies that $l_{r}\left(A^{m}\right) \leqslant 3$ (for any $m, A^{m}$ is certainly $\alpha$-reduced). This case therefore reduces to the word problem for $G_{0}$.

So suppose that $D$ and $E$ are symmetric with maximal representations $\left(\Delta, \Pi, X_{u}\right)$ and $\left(\Omega, \Phi, Y_{u}\right)$. To be conjugate by $A^{m}, D$ and $E$ must be $q$-parallel and in this case, $A^{-m} D A^{m}={ }_{G_{0}} E$ if and only if $A^{m}$ specifies a proof that $\Delta q \Pi=\tau_{*} \Omega q \Phi$ and, for each $u$, $X_{u}={ }_{H_{2}} Y_{u}$. (Theorem 8 is applicable since $A^{m}$ is $\alpha$-reduced.) It suffices then to determine whether or not there exists $m$ such that $A^{m}$ specifies a proof that $\Delta q \Pi=\mathfrak{I}_{*} \Omega q \Phi$.

Firstly we determine whether or not $A$ is proof-inducing (by Solution 8 , this can be done). If $A$ is not proof-inducing, then neither is $A^{m}$, for any $m>0$. So suppose that $A$ is proof-inducing; compute (recursively) $\Theta, \Psi, \Lambda$ and $\Xi$ from $A$ as per Lemma 49. If $A^{-m} D A^{m}={ }_{G_{0}} E$, then the situation of Lemma 50 occurs with $\Delta q \Pi$ as $\Delta_{0} q \Pi_{0}$ and $\Omega q \Phi$ as $\Delta_{m} q \Pi_{m}$. If either (i), (ii), (iv) or (v) holds, then there is an effectively computable upper bound for $m$ and we can fall back on the solution to the word problem. In case (iii), $D$ and $E$ are conjugate by $A^{m}$ if and only if $D=G_{6} E$.

$\S 5$. To complete the analysis we shall show that (? $Y, Z$ normal) $(\exists W) W^{-1} Y W={ }_{G_{0}} Z$ is recursively solvable. Call two words $Y$ and $Z$ divorced if $\left(\exists X q\right.$-free) $X^{-1} Y X={ }_{G_{0}} Z$ fails.

REDUCTION VIII. (? $Y, Z$ normal $)(\exists W) W^{-1} Y W={ }_{G_{0}} Z \longrightarrow(? Y, Z$ normal, divorced) $(\exists W) W^{-1} Y W={ }_{G_{0}} Z$ and $(? Y, Z)(\exists X) X^{-1} Y X={ }_{H} Z \cdot\left({ }^{1}\right)$

Proof. Immediate since $X^{-1} Y X={ }_{G_{0}} Z$ if and only if $X^{-1} Y X={ }_{H} Z$.

( $\left.{ }^{3}\right)$ It is expositionally convenient to consider this problem rather than (? $Y, Z$ normal) $(\exists X)$ $X^{-1} Y X={ }_{H} Z$. 
We examine the problem $(? Y, Z)(\exists X) X^{-1} Y X={ }_{H} Z$ first. Our approach is similar to that taken in $\S 2$. Define $H_{4}=\left(x, t, k, r_{i} ; t x=x t, k x=x k, t r_{i}=r_{i} t, k r_{i}=r_{i} k\right)$ and $H_{5}=$ $(x, t, k ;: ; x=x t, k x=x k)$.

LEMMA 51. (i) $\operatorname{Cond}_{J L B}\left(H_{5}, F(x),\{t, k\}\right)$ holds. (ii) $\operatorname{Cond}_{J L B}\left(H_{4}, H_{5}, r_{i}\right)$ holds. (iii) $\operatorname{Cond}_{J L B}\left(H, H_{4}, s_{b}\right)$ holds.

Proof. The various isomorphism conditions are easily verified.

THEOREM 9. Let s-contraction be w.r.t. $\left(H, H_{4}\right)$. Then $(\exists X) X^{-1} Y X={ }_{H} Z$ if and only if $(\exists X) X^{-1} \bar{s}\{Y\} X={ }_{H} \bar{s}\{Z\}$.

Proof. By GL 2 and Lemma 51.

Тн ЕОR Е 10 . Let s-contraction be w.r.t. $\left(H, H_{4}\right)$ and r-contraction w.r.t. $\left(H, H_{2}\right)$. Then (i) $(\exists X) X^{-1} Y X={ }_{H} Z$ if and only if $(\exists X) X^{-1} \bar{r}\{Y\} X={ }_{H} \bar{r}\{Z\}$; (ii) if $Y$ is s-contracted, then $\bar{r}\{Y\}$ is s-contracted.

Proof. (i) By GL 2 and Lemma 19. (ii) This is proved by a routine argument similar to that given for part (ii) of Theorem 2.

ТH.эОRЕM 11. Let s-contraction be w.r.t. $\left(H, H_{4}\right)$, r-contraction w.r.t. $\left(H, H_{2}\right)$ and tkcontraction w.r.t. $\left(H, G_{3}\right)$. Then (i) $(\exists X) X^{-1} Y X={ }_{H} Z$ if and only if $(\exists X) X^{-1} \bar{t} \bar{k}\{Y\} X={ }_{H}$ $\overline{t k}\{Z\}$; (ii) if $Y$ is s-contracted and r-contracted, then $\overline{t k}\{Y\}$ is s-contracted and r-contracted.

Proof. (i) By GL 2 and Lemma 18. (ii) Again a routine argument similar to that given for part (ii) of Theorem 2 is required.

Call a word $Y$ hypernormal if it is $s$-contracted w.r.t. $\left(H, H_{4}\right)$, r-contracted w.r.t. $\left(H, H_{2}\right)$ and $t k$-contracted w.r.t. $\left(H, G_{3}\right)$.

REDUCTION IX. $(? Y, Z)(\exists X) X^{-1} Y X={ }_{H} Z \longrightarrow(? Y, Z$ hypernormal $)(\exists X) X^{-1} Y X={ }_{H} Z$.

Procf. It is not hard to see that there is a recursive procedure for computing $t k\{r\{s\{Y\}\}\}$ and $t k\{r\{s\{Z\}\}\}$. The reduction follows from Theorems 9-11.

Call a word $Y$ r-regular if $Y$ is hypernormal and has $r_{i}^{-1}$ as its final symbol. Call $Y$ orthodox if $Y$ is $r$-regular and is not $s t k$-free (i.e. $Y$ is either not $s$-free or not $t$-free or not $k$-free).

LEMMA 52. $\operatorname{Cond}_{J_{L B}}\left(H, F\left(x, r_{t}\right),\left\{s_{b}, t, k\right\}\right)$ holds.

Proof. It is easy to verify that $\{x\},\left\{r_{i}\right\},\left\{x^{2}\right\}$ and $\left\{x r_{i} x\right\}$ are sets of free generators in $F\left(x, r_{i}\right)$. Once again we do not verify the strong isomorphism condition.)

REIUCTION X. (? $Y, Z$ hypernormal $)(\exists X) X^{-1} Y X={ }_{H} Z \rightarrow(? Y, Z$ orthodox $)\left(\exists C_{1}\right)$ 
$\operatorname{Conj}\left(Y, Z, C_{1}, H, F\left(x, r_{i}\right)\right.$, stk) and $\left(? R, R^{\prime}\right.$ r-regular $)\left(\exists C_{1}\right) C_{1}^{-1} R C_{1}={ }_{H} R^{\prime}$ and $(? L, M$ hypernormal $)(\exists X) X^{-1} L X={ }_{H} M$.

Proof. By GL 3 and GL 7, we can reduce to the problems ( $Y, Z r$-regular)( $\left.\exists C_{1}\right)$ $C_{1}^{-1} Y C_{1}={ }_{H} Z$ and (? $L, M$ hypernormal) $(\exists X) X^{-1} L X={ }_{H} M$. Since if $C_{1}$ is $\alpha$-reduced, then $C_{1}$ is stk-reduced, the complete reduction follows from GL 4 and GL 5.

Let $Y$ be an orthodox word; we define $\eta$-reduction in a manner analogous to the definition of $z$-reduction. If $Y$ is $Y^{\prime} t^{-\varepsilon} R r_{i}^{-1}$, then $\eta_{1}[Y]$ is $t^{-\varepsilon} Y^{\prime} R r_{i}^{-1}$. If $Y$ is $Y^{\prime} k^{-\varepsilon} R r_{i}^{-1}$, then $\eta_{1}[Y]$ is $k^{-\varepsilon} Y^{\prime} R r_{i}^{-1}$. If $Y$ is $Y^{\prime} s_{b}^{-1} R r_{i}^{-1}$ and $\left(\exists T_{1}\right)\left[R=T_{1}\right.$ in $F\left(x, r_{i}\right)$ and $T_{1} r_{i}^{-1}$ is $\alpha$-reduced], then $\eta_{1}[Y]$ is $x^{-1} s_{b}^{-1} Y^{\prime} T_{-1} x^{-1} r_{i}^{-1}$. If $Y$ is $Y^{\prime} s_{b} R r_{i}^{-1}$ and $\left(\exists T_{-1}\right)\left[R=T_{-1} x^{-1}\right.$ in $F\left(x, r_{i}\right)$ and $T_{-1} x^{-1} r_{i}^{-1} x^{-1}$ is $\alpha$-reduced], then $\eta_{1}[Y]$ is $s_{b} x Y^{\prime} T_{1} r_{i}^{-1}$. If $Y$ does not satisfy any of the above conditions, define $\eta_{1}[Y]$ to be $Y$. Define $\eta_{n}[Y]$ to be $\eta_{1}\left[\eta_{n-1}[Y]\right]$ and $\eta_{0}[Y]$ to be $Y$.

We abbreviate Conj $\left(Y, Z, C_{1}, H, F\left(x, r_{i}\right), s t k\right)$ to $\operatorname{Conj}\left(Y, Z, C_{1}, s t k\right)$.

REDUCTION XI. (? Y,Z orthodox) $\left(\exists C_{1}\right)$ Conj $\left(Y, Z, C_{1}\right.$, stk) $\longrightarrow(? Y, Z$ orthodox, stk-parallel $)\left(\exists C_{1}\right)$ Conj $\left(Y, Z, C_{1}, s t k\right)$.

Proof. The argument required exactly parallels that given for Reduction VI. In particular, analogues of Lemmas 39-41 are needed, plus the fact that computing $\eta_{n}[Y]$ is a recursive process.

Reduction XII. (? $Y, Z$ orthodox, stk-parallel $)\left(\exists C_{1}\right) \operatorname{Conj}\left(Y, Z, C_{1}, s t k\right) \longrightarrow\left(? R, R^{\prime}\right.$, $C_{1} ; R, R^{\prime}$ r-regular) $(\exists m) C_{1}^{-m} R C_{1}^{m}={ }_{H} R^{\prime}$.

Proof. The argument required parallels that given for Reduction VII. Analogues of Lemmas $44-47$ are needed, plus the fact that computing $\eta_{n}[Y]$ is a recursive process.

LEMMA 53. Let $R$ and $R^{\prime}$ be $\alpha$-reduced words where $R$ is $x^{m_{1}} r_{i_{1}}^{\varepsilon_{1}} x^{m_{2}} \ldots x^{m_{p} r_{i}^{\varepsilon_{p}}}$ and $R^{\prime}$ is $x^{n_{1}} r_{i_{1}}^{\varepsilon_{2}} x^{n_{2}} \ldots x^{n_{p}} r_{i_{p} \varepsilon_{p}}$ and let $C_{-\varepsilon_{p}}$ have s-signature $\sigma$. If $C_{-\varepsilon_{p}}^{-1} R C_{-\varepsilon_{p}}={ }_{H} R^{\prime}$, then $n_{k}=2^{\sigma} m_{k}+$ $\left(2^{\sigma}-1\right)\left(\varepsilon_{k-1}+\varepsilon_{k}\right), k=1,2, \ldots, p$ (writing $\left.\varepsilon_{p}=\varepsilon_{1-1}\right)$.

Proof. The lemma is proved by a straightforward induction on $l_{s t k}\left(C_{-\varepsilon_{p}}\right)$.

Soldton 10. (? $R, R^{\prime}, C_{1} ; R, R^{\prime}$ r-regular) $(\exists m) C_{1}^{-m} R C_{1}^{m}={ }_{H} R^{\prime}$ is recursively solvable.

Proof. We need only consider $R$ and $R^{\prime}$ when they are $\alpha$-reduced and $r$-parallel. Let $R$ be $x^{m_{1}} r_{i_{1}}^{\varepsilon_{1}} x^{m_{2}} \ldots x^{m_{p}} r_{i_{p}}^{-1}$ and $R^{\prime}$ be $x^{n_{1}} r_{i_{1}}^{\varepsilon_{1}} x^{n_{2}} \ldots x^{n_{p}} r_{i_{p}}^{-1}$. If $C_{1}$ has $s$-signature $\sigma$, then $C_{1}^{m}$ has $s$-signature $m \sigma$. If $\sigma \neq 0$, then $C_{1}^{-m} R C_{1}^{m}={ }_{H} R^{\prime}$ only if, for each $k, n_{k}=2^{m \sigma} m_{k}+$ $\left(2^{m \sigma}-1\right)\left(\varepsilon_{k-1}+\varepsilon_{k}\right)$. There is at most one possible integral value of $m$ which satisfies these equations and thus we may fall back on the solution to the word problem for $H$. 
If $\sigma=0$, then $C_{1}^{-m} R C_{1}^{m}={ }_{H} R^{\prime}$ only if, for each $k, n_{k}=m_{k}$, i.e. only if $R={ }_{H} R^{\prime}$. Again we may fall back on the word problem.

We have now dealt completely with the problem (? $Y, Z$ orthodox) $\left(\exists C_{1}\right) \operatorname{Conj}(Y$, $\left.Z, C_{1}, s t k\right)$. The next one to be examined is $\left(R, R^{\prime}\right.$ r-regular $)\left(\exists C_{1}\right) C_{1}^{-1} R C_{1}={ }_{H} R^{\prime}$.

SOLUTION 11. (R, $R^{\prime}$ r-regular $)\left(\exists C_{1}\right) C_{1}^{-1} R C_{1}={ }_{H} R^{\prime}$ is recursively solvable.

Proof. If $R$ and $R^{\prime}$ are not $r$-parallel, then they are not conjugate by a $C_{1}$. So let $R$ and $R^{\prime}$ be as in Lemma 53 with $\varepsilon_{p}=-1$. If $\left(\exists C_{1}\right) C_{1}^{-1} R C_{1}={ }_{H} R^{\prime}$, then $(\exists \sigma)\left[n_{k}=m_{k} 2^{\sigma}+\right.$ $\left(2^{\sigma}-1\right)\left(\varepsilon_{k-1}+\varepsilon_{k}\right)$ for all $k$ ]. It is easily seen that at most one such $\sigma$ exists and we can certainly determine whether or not $\sigma$ is an integer. If no such integral $\sigma$ exists, then $\left(\exists C_{1}\right) C_{1}^{-1} R C_{1}={ }_{H} R^{\prime}$ fails.

Suppose such an integral $\sigma$ exists; let $C_{1}$ be $\left(s_{b} x\right)^{\sigma}$. It is easy to show that $C_{1}^{-1} R C_{1}={ }_{H} R^{\prime}$.

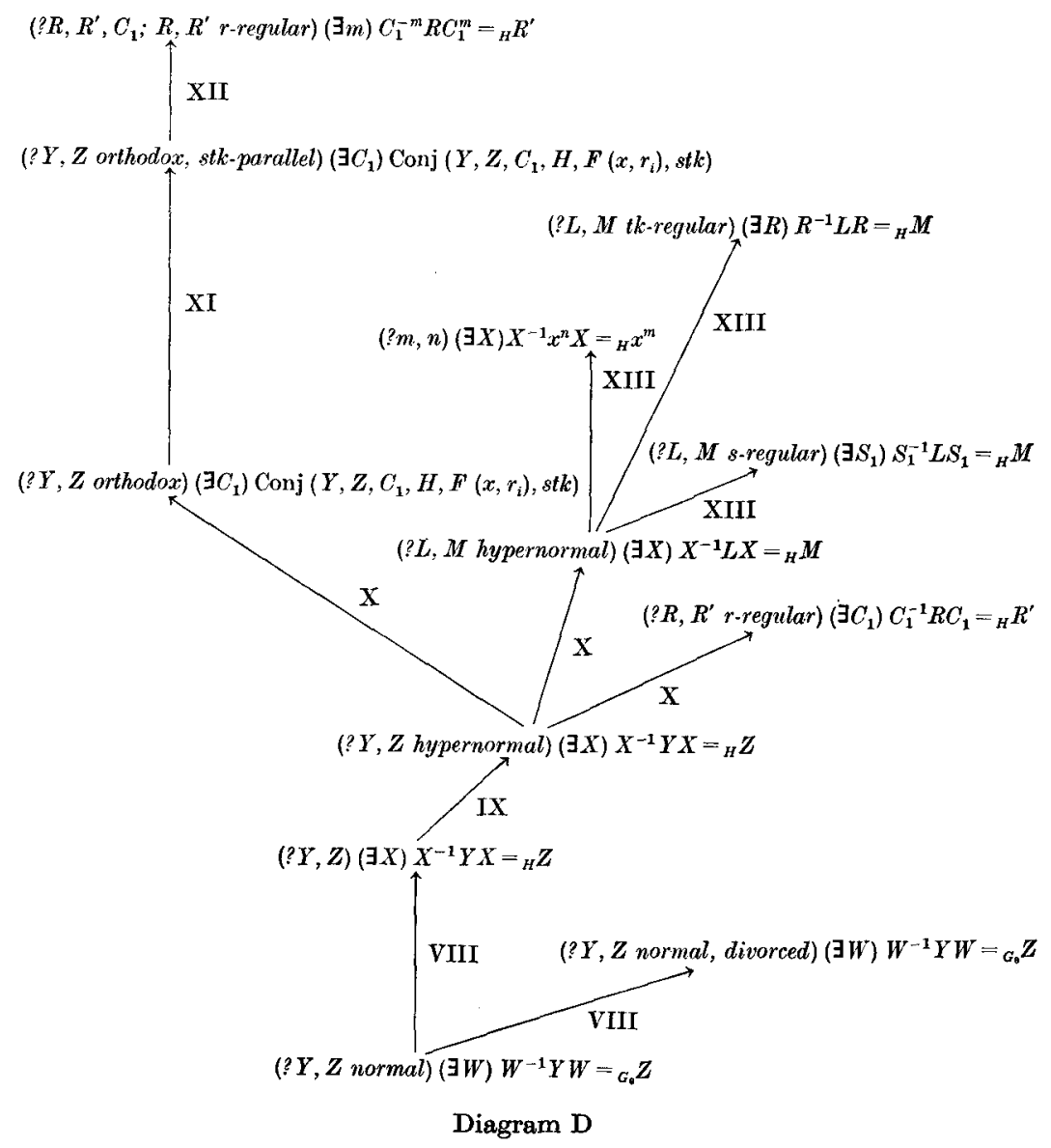


Call a word $L t k$-regular if $L$ is hypernormal and $L$ terminates in $t^{-1}$ or $k^{-1}$. Call a word $L s$-regular if $L$ is hypernormal and $L$ terminates in $s_{b}^{-1}$.

Reduction XIII. (?L, $M$ hypernormal $)(\exists X) X^{-1} L X={ }_{H} M \longrightarrow(? L, M$ tk-regular $)(\exists R)$ $R^{-1} L R={ }_{H} M$ and (?L, $M$ s-regular $)\left(\exists S_{1}\right) S_{1}^{-1} L S_{1}={ }_{H} M$ and $(? m, n)(\exists X) X^{-1} x^{n} X={ }_{H} x^{m}$.

Proof. Cond ${ }_{J L B}\left(H, G_{3},\{t, k\}\right)$ and $\operatorname{Cond}_{J L B}\left(H, H_{4}, s_{b}\right)$ hold. The reduction follows from GL 3 and GL 7.

Let $I$ be a word on $k, t$ and $x$; if $I$ contains $d$ positive $x$-symbols and $b$ negative $x$-symbols, call $\varrho=d-b$ the $x$-signature of $I$. If $L$ is $I_{0} s_{b_{1}}^{\varepsilon_{1}} I_{1} \ldots I_{r-1} s_{b_{r}}^{\varepsilon} I_{r}$ and $\varrho_{k}$ is the $x$-signature of $I_{k}$, the $(r+1)$-tuple $\left(\varrho_{0}, \varrho_{1}, \ldots, \varrho_{r}\right)$ is the $x$-deployment of $L$.

LEMMA 54. Let $L$ and $M$ be stk-reduced, stk-parallel words with $x$-deployments $\left(\lambda_{0}\right.$, $\left.\lambda_{1}, \ldots, \lambda_{r}\right)$ and $\left(\varrho_{0}, \varrho_{1}, \ldots, \varrho_{r}\right)$ and (common) s-distribution $\left(\sigma_{0}, \sigma_{1}, \ldots, \sigma_{r}\right)$. If $x^{-n} L x^{m}={ }_{H} M$, then $n=2^{-o_{r}} m+\sum_{k=0}^{r} 2^{-\sigma_{k}}\left(\lambda_{k}-\varrho_{k}\right)$.

Proof. By induction w.r.t. $l_{\text {stk }}(L)$.

LEMMA 55. Let $L$ and $M$ be as in Lemma 54 with $\sigma_{r} \neq 0$. If $x^{-m} L x^{m}={ }_{H} M$, then $m=$ $\left(1-2^{-\sigma_{r}}\right)^{-1} \sum_{k=0}^{r} 2^{-\sigma_{k}}\left(\lambda_{k}-\varrho_{k}\right)$

Proof. Immediate from Lemma 54.

Soldtion 12. (?L, $M$ stk-reduced) $(\exists m) x^{-m} L x^{m}={ }_{H} M$ is recursively solvable.

Proof. We need only consider $L$ and $M$ if they are $s t k$-parallel. If $\sigma(L) \neq 0$, then by Lemma 55 there is at most one possible value for $m$ and this value may be recursively computed. The problem is thus reducible to the word problem.

Suppose $\sigma(L)=0$; let $L$ have $s$-index $\nu$. By Lemma 28, $(\exists m) x^{-m} L x^{m}={ }_{H} M$ only if $(\exists m)\left[|m|<2^{v}\right.$ and $\left.x^{-m} L x^{m}={ }_{H} M\right]$. Again we can fall back on the word problem.

L⿴Mм A 56. Let $L$ and $M$ be stk-reduced, stk-parallel words such that $(\exists m) x^{-m} L x^{m}={ }_{H} M$ fails and let $C_{1}^{*}$ and $C_{-1}^{*}$ be stk-parallel to $L$. Then $(\exists R) R^{-1} L R={ }_{H} M$ if and only if either

(1) $(\exists c)(\exists d)\left[x^{-c} L x^{c}={ }_{H} C_{1}^{*}\right.$ and $\left.x^{-d} M x^{d}={ }_{H} C_{-1}^{*}\right]$ or

(2) $(\exists c)(\exists d)\left[x^{-c} L x^{c}={ }_{H} C_{-1}^{*}\right.$ and $\left.x^{-d} M x^{d}={ }_{H} C_{1}^{*}\right]$.

Proof. Suppose that $R^{-1} L R={ }_{H} M$; we may assume that $R$ is $\alpha$-reduced and hence $r$-reduced w.r.t. $\left(H, H_{2}\right)$. Clearly $R$ cannot be $r$-free. Hence neither $R^{-1} L R$ nor $R M R^{-1}$ are $r$-reduced. There are four possible ways in which this could occur. These are (1) and (2) above along with 
(3) $(\exists c)(\exists d)\left[x^{-c} L x^{c}={ }_{H} C_{1}^{*}\right.$ and $\left.x^{-d} M x^{d}={ }_{H} C_{1}^{*}\right]$ and

(4) $(\exists c)(\exists d)\left[x^{-c} L x^{c}={ }_{H} C_{-1}^{*}\right.$ and $\left.x^{-d} M x^{d}={ }_{H} C_{-1}^{*}\right]$.

But (3) and (4) both imply that $x^{d-c} L x^{c-d}={ }_{H} M$ which is impossible.

Conversely suppose that (1) occurs; then $x^{d} r_{i}^{-1} x^{-c} L x^{c} r_{i} x^{-d}={ }_{H} M$. If (2) occurs, then $x^{d} r_{i} x^{-c} L x^{c} r_{i}^{-1} x^{-d}={ }_{H} M$.

SOLUTION 13. (i) (?L, $M$ tk-regular) $(\exists R) R^{-1} L R={ }_{H} M$ is recursively solvable;

(ii) (? $L, M$ s-regular) $\left(\exists S_{1}\right) S_{1}^{-1} L S_{1}={ }_{H} M$ is recursively solvable.

Proof. (i) This is an easy consequence of Solution 12 and Lemma 56. (ii) Since $S_{1}$ has the same range as $R$ this also follows from Solution 12 and Lemma 56.

Our penultimate problem is $(? m, n)(\exists X) X^{-1} x^{n} X={ }_{H} x^{m}$.

LEMMA 57. If $X^{-1} x^{n} X={ }_{H} x^{m}$ and $X$ is r-reduced, then either $X$ is $r$-free or $m=n=0$.

Proof. Suppose that $m \neq n$ and that $X$ is not $r$-free. Then $(\exists L)\left(\exists C_{\varepsilon}\right) L^{-1} x^{n} L={ }_{H_{z}} C_{\varepsilon}$. Then $s t k\left[L^{-1} x^{n} L\right]={ }_{H_{2}} \alpha\left[C_{\varepsilon}\right]$ whence it follows that $\alpha\left[C_{\varepsilon}\right]$ is 1 . Thus $L^{-1} x^{n} L={ }_{H_{3}} 1$ and hence $n=0$. In a similar way we can prove that $m=0$ which is impossible. The lemma follows from this.

Soldtion 14. $(? m, n)(\exists X) X^{-1} x^{n} X={ }_{H} x^{m}$ is recursively solvable.

Proof. By Lemma 57, it clearly suffices to solve the problem $(? m, n)(\exists L) L^{-1} x^{n} L={ }_{H a} x^{m}$. But it is easy to show that $(\exists L) L^{-1} x^{n} L={ }_{H_{2}} x^{m}$ if and only if $(\exists \sigma) m=2^{\sigma} n$.

Finally we provide a recursive solution to the problem (? $Y, Z$ normal, divorced $)(\exists W)$ $W^{-1} Y W={ }_{G_{0}} Z$.

LEMMA 58. Let $Y$ and $Z$ be normal and suppose that $Y$ and $Z$ are not both tk-free. If $(\exists W) W^{-1} Y W={ }_{G_{0}} Z$, then there exist circular variants $Y_{0}$ and $Z_{0}$ of $Y$ and $Z$ respectively which are not divorced.

Proof. By GL 3 and Lemma 12.

RedUCtion XIV. (? $Y, Z$ normal, divorced) $(\exists W) W^{-1} Y W={ }_{G_{0}} Z \longrightarrow(? F, G$ normal, divorced $)(\exists W) W^{-1} F^{\prime} W={ }_{G_{0}} G$.

Proof. Let $Y$ and $Z$ be normal and divorced. Suppose that $Y$ and $Z$ are not both $t k$-free. If $(\exists W) W^{-1} Y W={ }_{G_{0}} Z$, then, by Lemma 58, $Y$ and $Z$ have circular variants which are not divorced. But this means that $Y$ and $Z$ are not divorced.

LEMM A 59. Let $F$ and $G$ be normal and divorced. Then $(\exists W) W^{-1} F^{\prime} W={ }_{G_{0}} G$ if and only if either (a) $\left(\exists F_{0}\right)\left(\exists G_{0}\right)(\exists A)(\exists B)\left[F_{0}^{-1} A F_{0}={ }_{G_{8}} F\right.$ and $G_{0}^{-1} B G_{0}={ }_{G_{3}} G$ and $A$ and $B$ are $r$-circum- 
parallel to $\left.F^{\prime}\right]$. or (b) $\left(\exists F_{0}\right)\left(\exists G_{0}\right)(\exists A)(\exists B)\left[F_{0}^{-1} B F_{0}={ }_{G_{3}} F\right.$ and $G_{0}^{-1} A G_{0}=G_{G_{3}} G$ and $A$ and $B$ are r-circumparallel to $F$ ].

Proof. Let $W^{-1} F W={ }_{G_{0}} G$; since $F$ and $G$ are $t k$-free, it follows that we may suppose that $W$ is $t k$-free. Also we may suppose that $W$ is $q$-reduced. Since by assumption $W$ is not $q$-free, we can write $W$ as $H_{1} q^{\varepsilon_{1}} W^{\prime} q^{\varepsilon_{n}} H_{2}$ (as $H_{1} q^{\varepsilon} H_{2}$ if $W$ has only one $q$-symbol).

It is clear that $W^{-1} F W$ and $W G W^{-1}$ are not $q$-reduced. Four possible cases occur; these are as follows.

(1) $\varepsilon_{1}=1=\varepsilon_{n}$, Then $\left(\exists A^{*}\right) H_{1}^{-1} F H_{1}={ }_{H} A^{*}$ and $\left(\exists B^{*}\right) H_{2} G H_{2}^{-1}={ }_{H} B^{*}$ and we may take $A^{*}$ and $B^{*}$ to be $\alpha$-reduced. (Notice that it is not necessarily the case that $A^{*} \sim B^{*}$.) Write $A^{*}$ as $A^{\prime-1} A A^{\prime}$ where $A^{\prime}$ is maximal. Similarly write $B^{*}$ as $B^{\prime-1} B B^{\prime}$. Then $A$ and $B$ are $r$-contracted.

Now $H_{1}^{-1} F H_{1}={ }_{G_{3}} A^{*}$ and $H_{2} G H_{2}^{-1}={ }_{G_{3}} B^{*}$ whence $A^{\prime} H_{1}^{-1} F H_{1} A^{\prime-1}={ }_{G s} A$ and $B^{\prime} H_{2} G H_{2}^{-1} B^{\prime-1}={ }_{G y} B$. This implies that $A$ is $r$-circumparallel to $F$ and $B$ is $r$-circumparallel to $G$. Since $F$ and $G$ must be $r$-circumparallel, the lemma follows by taking $F_{0}$ to be $A^{\prime} H_{1}^{-1}$ and $G_{0}$ to be $B^{\prime} H_{2}$.

(2) $\varepsilon_{1}=-1=\varepsilon_{n}$. This gives rise to (b) in the same way as (1) gives rise to (a).

(3) $\varepsilon_{1}=1, \varepsilon_{n}=-1$. We want to show that this is impossible. By Britton's Lemma, we obtain $\left(\exists A^{*}\right) H_{1}^{-1} F^{*} H_{1}={ }_{G_{3}} A^{*}$ and $\left(\exists A^{\sim}\right) H_{2} G H_{2}^{-1}={ }_{G_{3}} A^{\sim}$. By an analysis similar to that of (1), we obtain $F_{0}^{-1} A F_{0}={ }_{G_{3}} F$ with $A$ r-circumparallel to $F$ and $G_{0}^{-1} A^{\prime} G_{0}={ }_{G_{3}} G$ with $A^{\prime}$ $r$-circumparallel to $G$. Then $A$ and $A^{\prime}$ must be $r$-circumparallel whence $\left(\exists A^{\prime \prime}\right) A^{\prime \prime-1} A A^{\prime \prime}={ }_{G s} A^{\prime}$. Hence $G={ }_{G_{3}} G_{0}^{-1} A^{\prime} G_{0}={ }_{G_{3}} G_{0}^{-1} A^{\prime \prime}-1 F_{0} F F_{0}^{-1} A^{\prime \prime} G_{0}$ which means that $Y$ and $Z$ are not divorced.

(4) $\varepsilon_{1}=-1, \varepsilon_{n}=1$. In the same way as (3), this is impossible.

Conversely suppose that (a) holds. Since $A$ and $B$ are $r$-circumparallel to $F$, there exist words $A^{\prime}$ and $A^{*}$ such that $A^{*}$ is $r$-parallel to $B$, i.e. $A^{*} \sim B$, and $A^{\prime-1} A A^{\prime}={ }_{G_{s}} A^{*}$. Then it follows that $\left(G_{0}^{-1} q^{-1} A^{\prime-1} F_{0}\right) F\left(F_{0}^{-1} A^{\prime} q G_{0}\right)={ }_{G_{0}} G$. In a similar way if (b) holds, we can show that $F$ and $G$ are conjugate.

Solution 15. (?F, G normal, divorced $)(\exists W) W^{-1} F W={ }_{G_{0}} G$ is recursively solvable.

Proof. It follows from Lemma 59 that the given problem is reducible to the problem $(? F, G)(\exists H) H^{-1} F H={ }_{G_{3}} G$. We have proved that $(? Y, Z)(\exists X) X^{-1} Y X={ }_{H} Z$ is recursively solvable. Since $(\exists X) X^{-1} F X={ }_{H} G$ if and only if $(\exists H) H^{-1} F H={ }_{G_{3}} G$, the result is immediate.

\section{Part V. Result B}

Proof of Result $B$. Let $\mathfrak{T}_{*}$ satisfy the assertions of the corollary to Shepherdson's result. Let $G\left(\left\{D_{n}\right\}, D\right)$ be $G_{0}$ 
(1) Let $\Omega_{n} q \Phi_{n}$ be such that $(? \Omega q \Phi) \Omega q \Phi=\mathfrak{T}_{*} \Omega_{n} q \Phi_{n}$ has degree $D_{n}$. Let $V_{n}$ be $\bar{\Omega}_{n}^{-1} t^{-1} \bar{\Omega}_{n} q \Phi_{n} t \Phi_{n}^{-1} q^{-1}$. Let $U$ be given; firstly compute $t k\{q\{r\{U\}\}\}$ and call this $U^{*}$. If $U$ and $V_{n}$ are to be conjugate, then $U^{*}$ must be $r$-free and contain exactly two $q$-symbols which are of opposing sign (by GL 3 and Theorems 1-3 since $V_{n}$ is normal). So suppose that $U^{*}$ is $r$-free and contains a single $q$ and a single $q^{-1}$. Then $U^{*}$ has a unique cyclic permutation $U^{\sim}$ of form $L_{1} q L_{2} q^{-1}$. Again using GL 3, $U^{*}$ and $V_{n}$ are conjugate if and only if either

(i) $(\exists A) A^{-1} U^{\sim} A={ }_{G_{0}} V_{n}$ or

(ii) $(\exists B) B^{-1} L_{2} q^{-1} L_{1} q B={ }_{G_{0}} \Phi_{n} t \Phi_{n}^{-1} q^{-1} \bar{\Omega}_{n}^{-1} t^{-1} \bar{\Omega}_{n} q$.

But it follows from Lemma 22 and its unstated dual that (i) is in fact equivalent to (ii).

There is a recursive procedure to determine whether or not $U^{\sim}$ is symmetric. If $U^{\sim}$ is not symmetric, then by Theorems $4-7$ and the solution to the word problem there is a recursive procedure to determine whether or not (i) holds. So suppose $U^{-}$is symmetric. Let it have maximal representation $\left(\Omega, \Phi, X_{1}, X_{2}\right)$; this is recursively computable. By Theorem 8, (i) holds if and only if $\Omega q \Phi=\widetilde{I}_{*} \Omega_{n} q \Phi_{n}$ and $X_{1}={ }_{H_{2}} t^{-1}$ and $X_{2}={ }_{H_{3}} t$ ( $V_{n}$ is symmetric with maximal representation $\left.\left(\Omega_{n}, \Phi_{n}, t^{-1}, t\right)\right)$. Hence the individual conjugacy problem for $V_{n}$ in $G_{0}$ is reducible to the problem (? $\left.q \Phi\right) \Omega q \Phi=\mathfrak{x}_{*} \Omega_{n} q \Phi_{n}$. The converse reduction follows from Lemmas 14,15 and 16 which prove that $\Omega q \Phi=\widetilde{r}_{*} \Omega_{n} q \Phi_{n}$ if and only if $t^{-1} \Phi^{-1} q^{-1} \bar{\Omega}^{-1} t \bar{\Omega} q \Phi$ and $t^{-1} \Phi_{n}^{-1} q^{-1} \bar{\Omega}_{n}^{-1} t \bar{\Omega}_{n} q \Phi_{n}$ are conjugate. The latter holds if and only if $\bar{\Omega}^{-1} t \bar{\Omega} q \Phi t^{-1} \Phi^{-1} q^{-1}$ and $\bar{\Omega}_{n}^{-1} t \bar{\Omega}_{n} q \Phi_{n} t^{-1} \Phi_{n}^{-1} q^{-1}$ are conjugate.

(2) Our whole argument in Parts I-IV shows that the word problem for special words of $\mathfrak{I}_{*}$ is Turing equivalent to the conjugacy problem for $G_{0}$.

(3) Let $V$ be any word of $G_{0}$; compute $t k\{q\{r\{V\}\}\}$ and call this $V^{*}$. There is a recursive procedure to determine whether or not $V^{*}$ has an $r$-free symmetric cyclic permutation. If no such cyclic permutation exists, then the individual conjugacy problem for $V$ in $G_{0}$ is solvable. (If $V^{*}$ is $q$-free, see $\S 5$ of Part IV; if $V^{*}$ is $r$-free but not $q$-free see Theorems 4-7; if $V^{*}$ is neither $r$-free or $q$-free see $\S 4$ of Part IV.) So suppose that $V^{*}$ has a cyclic permutation $V^{\sim}$ which is $r$-free and symmetric. Let $V^{\sim}$ have maximal representation $\left(\Omega, \Phi, Y_{u}\right)$. Then the individual conjugacy problem for $V$ in $G_{0}$ is Turing equivalent to the problem $(? \Delta q \Pi) \Delta q \Pi=\mathfrak{T}_{*} \Omega q \Phi$. To show this we argue as follows.

Let $U$ be given; if $t k\{q\{r\{U\}\}\}$ cannot be cyclically permuted into a symmetric word which is $q$-parallel to $V^{\sim}$, then there is a recursive procedure to determine whether or not $U$ and $V$ are conjugate. Otherwise let $U_{1}, U_{2}, \ldots, U_{m}$ be the collection of all symmetric cyclic permutations of $t k\{q\{r\{U\}\}\}$. Then $U$ and $V$ are conjugate if and only if $(\exists k)(\exists A) A^{-1} U_{k} A={ }_{G_{0}} V^{\sim}$. This last problem is reducible to $(? \Delta q \Pi) \Delta q \Pi=\mathfrak{T}_{*} \Omega q \Phi$.

Conversely let $\Delta q \Pi$ be given; let $U$ be an $r$-free word which is symmetric with maximal representation $\left(\Delta, \Pi, Y_{u}\right)$. Then $\Delta q \Pi=\mathfrak{T}_{*} \Omega q \Phi$ if and only if $(\exists A) A^{-1} U A={ }_{G_{0}} V^{\sim}$. 
To complete the reduction we must prove that $(\exists W) W^{-1} U W={ }_{G_{0}} V^{\sim}$ implies that ( $\exists A) A^{-1} U A=G_{G_{0}} V^{\sim}$. By GL 3 , there exist symmetric cyclic permutations $U_{0}$ and $V_{0}$ of $U$ and $V^{\sim}$ respectively and a word $A$ such that $A^{-1} U_{0} A={ }_{G_{0}} V_{0}$. Now $U_{0}$ and $V_{0}$ must have maximal representations $\left(\Delta, \Pi, Y_{j+1}, \ldots, Y_{n}, Y_{1}, \ldots, Y_{j}\right)$ and $\left(\Omega, \Phi, Y_{k+1}, \ldots, Y_{n}, Y_{1}, \ldots, Y_{k}\right)$. From Lemma 22, it follows that $A^{-1} U A=G_{0} V^{-}$.

\section{References}

[1]. AdJan, S. I., On algorithmic problems in effectively complete classes of groups (Russian). Dokl. Akad. Nauk SSSR, 123 (1958), 13-16.

[2]. BokUT', L. A., On a property of groups of Boone (Russian). Algebra i Logika Sem., 5 (1966), No. 5, 5-23; 6 (1967), No. 1, 15-24.

[3]. - - On groups of Novikov (Russian). Algebra i Logika Sem., 6 (1967), No. 1, 25-38.

[4]. Boone, W. W., Certain simple unsolvable problems of group theory. Indag. Math., 16 (1954), 231-237, 492-497; 17 (1955), 252-256, 571-577; 19 (1957), 22-27, 227-232.

[5]. — The word problem. Ann. of Math., 70 (1959), 207-265.

[6]. - - Word problems and recursively enumerable degrees of unsolvability. A first paper on Thue systems. Ann. of Math., 83 (1966), 520-571.

[7]. - Word problems and recursively enumerable degrees of unsolvability. A sequel on finitely presented groups. Ann. of Math., 84 (1966), 49-84.

[8]. - - Decision problems about algebraic and logical systems as a whole and recursively enumerable degrees of unsolvability. To appear in Proc. Kolloqium ü. Logik u. Grundlagen d. Math., Hannover, 1966.

[9]. Britton, J. L., The word problem. Ann. of Math., 77 (1963), 16-32.

[10]. Clapham, C. R. J., Finitely presented groups with word problems of arbitrary degrees of insolvability. Proc. London Math. Soc., Series 3, 14 (1964), 633-676.

[11]. Fridman, A. A., On the relation between the word problem and the conjugacy problem in finitely defined groups (Russian). Trudy Moskov. Mat. Obšč, 9 (1960), 329-365.

[12]. - Degrees of unsolvability of the problem of identity in finitely presented groups. Soviet Math., 3 (1962), Part 2, 1733-1737.

[13]. Friedrerg, R. M., Two recursively enumerable sets of incomparable degrees of unsolvability (solution of Post's problem, 1944). Proc. Nat. Acad. Sci. U.S.A., 43 (1957), $236-238$.

[14]. Lyndon, R. C. \& Schutzenberger, M. P., On the equation $a^{M}=b^{N} c^{P}$ in a free group, Michigan Math. J., 9 (1962), 289-298.

[15]. Miller III, C. F., Conjugacy and word problems in finitely generated groups, J. Symb. Logic, 32 (1967), 440-441.

[16]. - Characterisation of recursively enumerable degrees by conjugacy and word problems for finitely generated groups, J. Symb. Logic, 32 (19̀67), 441.

[17]. MUCnIK, A. A., Negative answer to the problem of reducibility of the theory of algorithms (Russian). Dokl. Akad. Nauk SSSR, 108 (1956), 194-197.

[18]. Novikov, P. S., Unsolvability of the conjugacy problem in the theory of groups (Russian). Izv. Akad. Nauk SSSR, Ser. Mat., 18 (1954), 485-524.

[19]. - On the algorithmic unsolvability of the word problem in group theory (Russian). Trudy Mat. Inst. Steklov, no. 44.

[20]. Rabin, M. O., Recursive unsolvability of group-theoretic problems. Ann. of Math., 67 (1958), 172-194.

[21]. Shaphernson, J. C., Machine configurations and word problems of given degree of unsolvability. Z. Math. Logik Grundlagen Math., 11 (1965). 149-175.

Received August 14, 1968 